Florida International University FIU Digital Commons

\title{
Effects of Repeated Readings on Reading Abilities of English Language Learners with Specific Learning Disabilities
}

Katrina G. Landa

Florida International University, katcin91@aol.com

DOI: $10.25148 /$ etd.FI09070601

Follow this and additional works at: https://digitalcommons.fiu.edu/etd

\section{Recommended Citation}

Landa, Katrina G., "Effects of Repeated Readings on Reading Abilities of English Language Learners with Specific Learning Disabilities" (2009). FIU Electronic Theses and Dissertations. 82.

https://digitalcommons.fiu.edu/etd/82 


\section{FLORIDA INTERNATIONAL UNIVERSITY}

Miami, Florida

EFFECTS OF REPEATED READINGS ON READING ABILITIES OF ENGLISH

\section{LANGUAGE LEARNERS WITH SPECIFIC LEARNING DISABILITIES}

A dissertation submitted in partial fulfillment of the

requirements for the degree of

DOCTOR OF EDUCATION

in

EXCEPTIONAL STUDENT EDUCATION

by

Katrina G. Landa 
To: Interim Dean Kingsley Banya

College of Education

This dissertation, written by Katrina G. Landa, and entitled Effects of Repeated Readings on Reading Abilities of English Language Learners with Specific Learning Disabilities, having been approved in respect to style and intellectual content, is referred to you for judgment.

We have read this dissertation and recommend that it be approved.

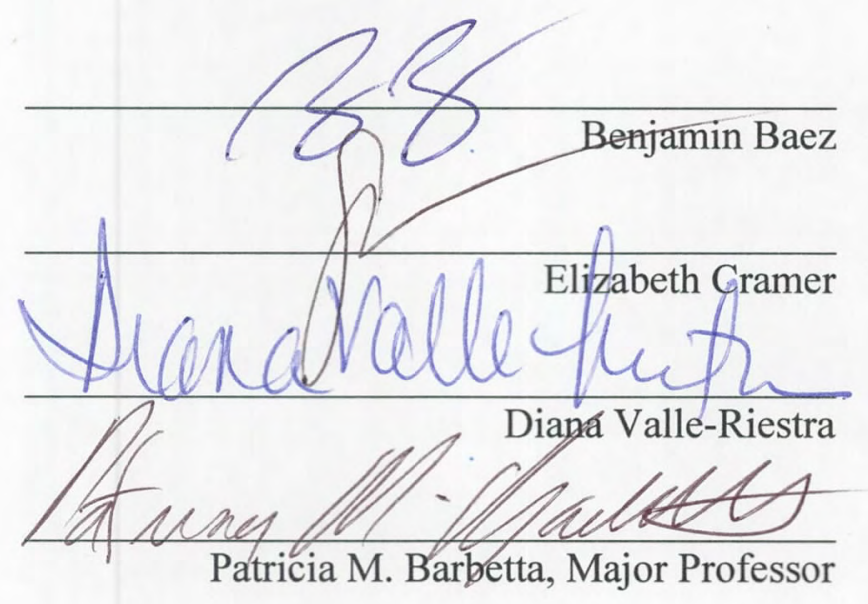

Date of Defense: May 26, 2009

The dissertation of Katrina G. Landa is approved.

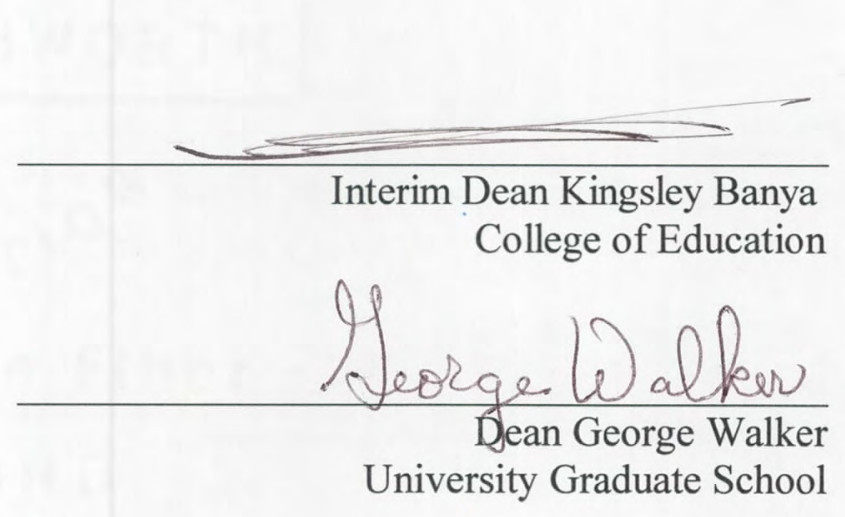

Florida International University, 2009 


\section{DEDICATION}

I dedicate this work to Cristian and Isabella. Thanks for taking this journey with me. 


\section{ACKNOWLEDGMENTS}

I would like to thank the members of my committee Major Professor Dr. Patricia M. Barbetta, Dr. Benjamin Baez, Dr. Elizabeth Cramer, and Dr. Diana Valle-Riestra for their honesty and support. This project would not have been possible without them.

I want to extend a special thanks to Dr. Ann Nevin, who first taught me about single subject design and whose enthusiasm taught me to love it. Also, I would like to thank Liliana Salazar and Lissette Robles for their support, input, and time dedicated to this study.

Throughout my tenure at FIU, I have found my fellow students, the faculty, and staff most supportive and thoughtful. I am grateful for everyone I have met while I worked on my doctorate and every experience this has entailed. 


\title{
ABSTRACT OF THE DISSERTATION \\ EFFECTS OF REPEATED READINGS ON READING ABILITIES OF ENGLISH \\ LANGUAGE LEARNERS WITH SPECIFIC LEARNING DISABILITIES
}

\author{
by
}

Katrina G. Landa

Florida International University, 2009

Miami, Florida

\section{Professor Patricia M. Barbetta, Major Professor}

This study investigated the effects of repeated readings on the reading abilities of

4, third-, fourth-, and fifth-grade English language learners (ELLs) with specific learning disabilities (SLD). A multiple baseline probe design across subjects was used to explore the effects of repeated readings on four dependent variables: reading fluency (words read correctly per minute; wpm), number of errors per minute (epm), types of errors per minute, and answer to literal comprehension questions. Data were collected and analyzed during baseline, intervention, generalization probes, and maintenance probes.

Throughout the baseline and intervention phases, participants read a passage aloud and received error correction feedback. During baseline, this was followed by fluency and literal comprehension question assessments. During intervention, this was followed by two oral repeated readings of the passage. Then the fluency and literal comprehension question assessments were administered. Generalization probes followed approximately $25 \%$ of all sessions and consisted of a single reading of a new passage at the same readability level. Maintenance sessions occurred 2-, 4-, and 6-weeks after the intervention ended. 
The results of this study indicated that repeated readings had a positive effect on the reading abilities of ELLs with SLD. Participants read more wpm, made fewer epm, and answered more literal comprehension questions correctly. Additionally, on average, generalization scores were higher in intervention than in baseline. Maintenance scores were varied when compared to the last day of intervention, however, with the exception of the number of hesitations committed per minute maintenance scores were higher than baseline means.

This study demonstrated that repeated readings improved the reading abilities of ELLs with SLD and that gains were generalized to untaught passages. Maintenance probes 2-, 4-, and 6- weeks following intervention indicated that mean reading fluency, errors per minute, and correct answers to literal comprehensive questions remained above baseline levels. Future research should investigate the use of repeated readings in ELLs with SLD at various stages of reading acquisition. Further, future investigations may examine how repeated readings can be integrated into classroom instruction and assessments. 


\section{TABLE OF CONTENTS}

CHAPTER

PAGE

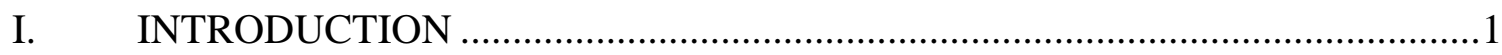

Purpose of the Study ……………………………………..............................

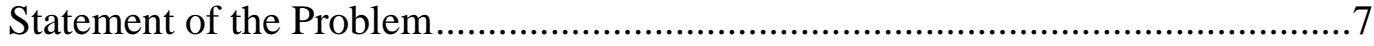

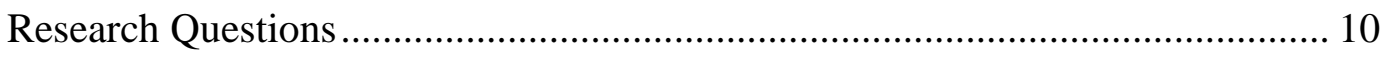

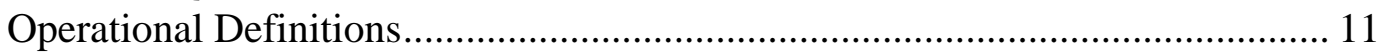

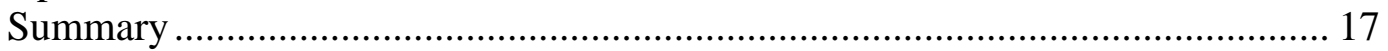

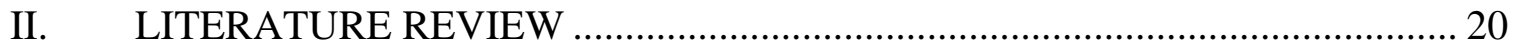

Characteristics of Students Who Do and Do Not Read Well ............................... 20

English Language Learners and Reading ........................................................ 22

Students with Specific Learning Disabilities and Reading................................... 24

English Language Learners with Specific Learning Disabilities and Reading..... 26

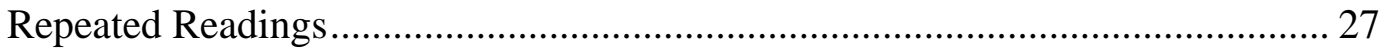

English Language Learners and Repeated Readings ........................................... 28

Students with Specific Learning Disabilities and Repeated Readings .................. 30

English Language Learners with Specific Learning Disabilities and

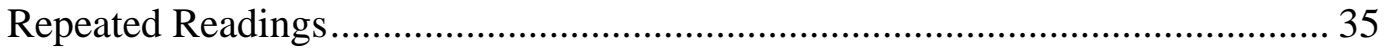

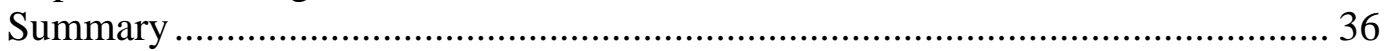

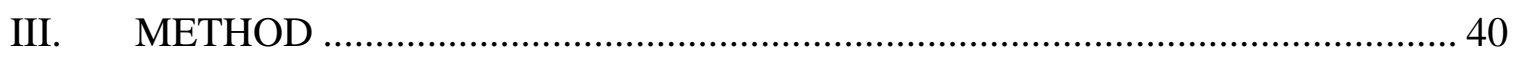

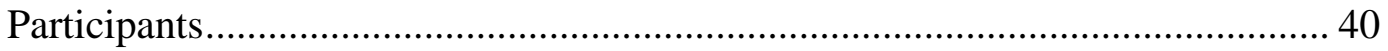

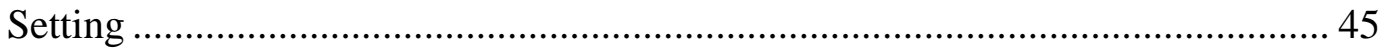

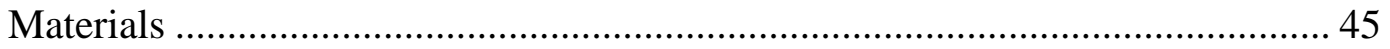

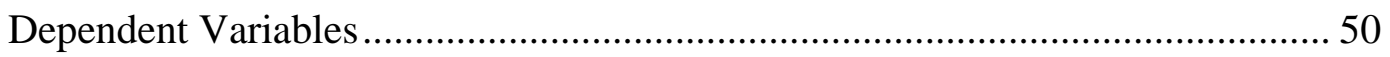

Interobserver Agreement ................................................................................ 52

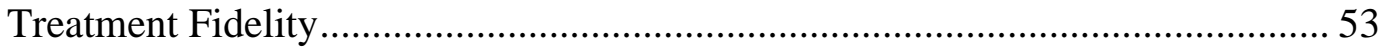

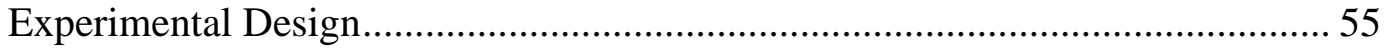

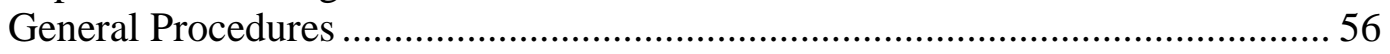

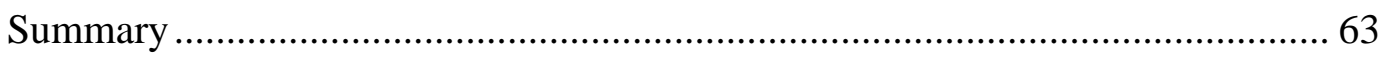

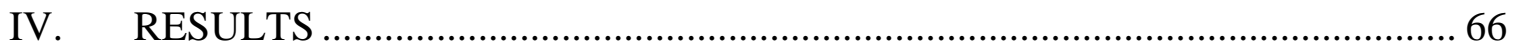

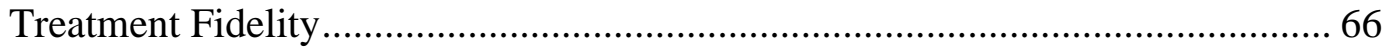

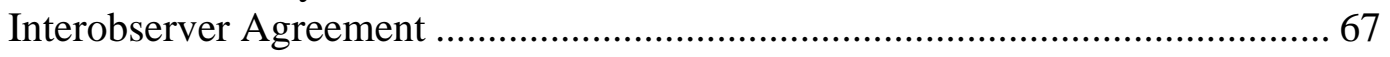

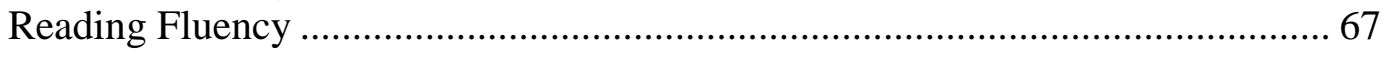

Number and Types of Errors Per Minute.......................................................... 77

Correct Answers to Literal Comprehension Questions ...................................... 115

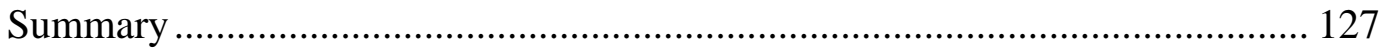

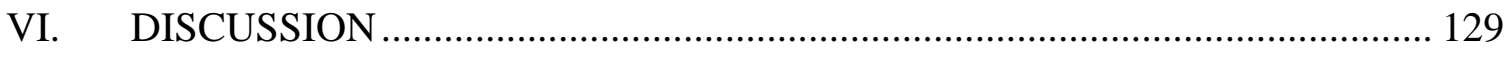

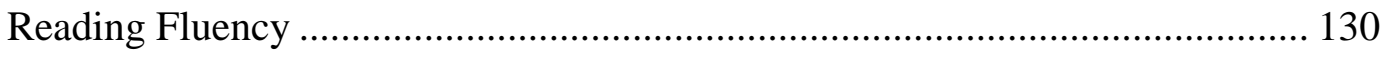

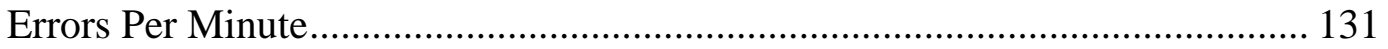


Types of Errors Per Minute............................................................................... 133

Correct Answers to Literal Comprehension Questions ................................... 134

Repeated Readings Summary ...................................................................... 135

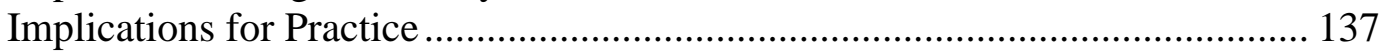

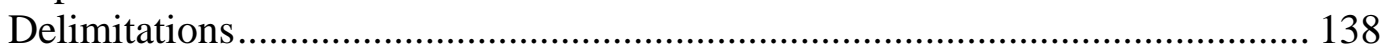

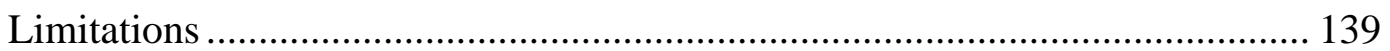

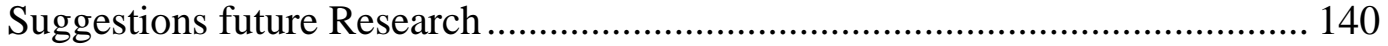

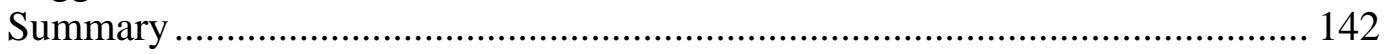

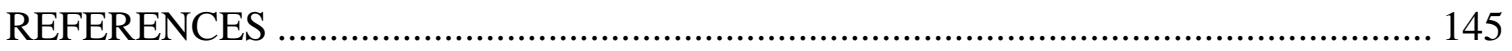

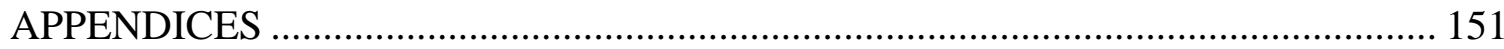

VITA 


\section{LIST OF TABLES}

TABLE

PAGE

1. Demographic and Analytical Reading Inventory Data ....................................43

2. Individual Means on Reading Fluency ....................................................... 70

3. Mean Reading Fluency in Comparison Sessions and Generalization Probes.........71

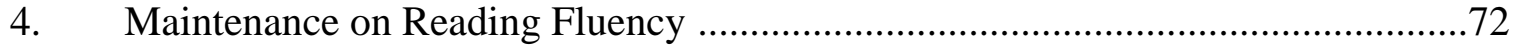

5. Individual Means on Number of Errors Per Minute ............................................8

6. Mean Errors Per Minute in Comparison Sessions and Generalization Probes.......82

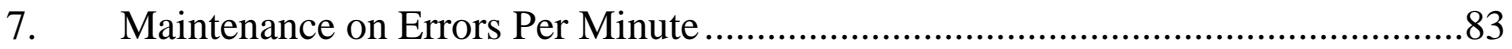

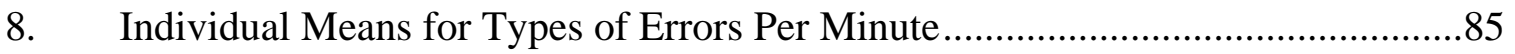

9. Mean Types of Errors Per Minute in Comparison Sessions and Generalization Probes

10. Maintenance Performance on Types of Errors Per Minute ................................90

11. Individual Means on Correct Answers to Literal Comprehension Questions. .....118

12. Mean Correct Answers to Literal Comprehension Questions in Sessions and Generalization Probes

13. Maintenance Performance on Correct Answers to Literal Comprehension Questions 


\section{CHAPTER I}

\section{INTRODUCTION}

Being able to read well is associated with both academic and social benefits. Academically, students who read well read fluently and with comprehension. They are able to summarize, interpret, and accept or reject the information on the printed page (Pressley, Gaskins, \& Fingeret, 2006). Students who read well are able to read long passages with ease and complete assignments in a reasonable time (Rasinski, 2000). These students typically experience success during the school years and are prepared to attend college or post-secondary programs following graduation (American College Testing Program, 2006). Socially, students who read well tend to be self-motivated. Therefore, they get more reading practice outside of the classroom simply because they find reading to be a pleasurable activity (Rasinski, 2000). Finally, economic success beyond the school years is associated with reading well in school (Brown \& Bogard, 2007; Snow, Burns, \& Griffin, 1998).

However, many students do not read well. For these readers, learning how to read fluently and with comprehension is a challenge. Nationally, there are an estimated 8 million fourth through $12^{\text {th }}$-grade students who read below grade level (U.S. Department of Education, 2005b). Statewide, 31\% of Florida's third-graders read below grade level (Florida Department of Education, 2008). In 2007, 37\% of third-grade students in the Miami-Dade County Public School district scored below grade level on the reading portion of the Florida Comprehensive Assessment Test (FCAT) and were considered to be struggling readers (Florida Department of Education, 2008). 
There are numerous negative academic and social outcomes correlated with an inability to read well (Hitchcock, Prater, \& Dowrick, 2004). Academically, students who do not read well cannot complete their assignments, and they are exposed to less information because they typically cannot read quickly. Students who do not read well may exhibit disruptive or withdrawn behaviors that distract them from learning activities (Hitchcock et al., 2004). The negative social outcomes correlated with an inability to read well include having a negative outlook and low participation in extracurricular school activities, lagging academically behind other students, and having a higher probability of dropping out of school (Lazarus \& Callahan, 2000). Beyond school, students who do not read well continue to experience negative social outcomes. They may have limited employment opportunities, a greater likelihood of living in poverty, and a higher rate of incarceration (Elbaum, Vaughn, Hughes, \& Moody, 2000; Hitchcock et al., 2004).

Students who are English language learners (ELLs) and those identified as having specific learning disabilities (SLD) are significantly at risk for the negative outcomes associated with not reading well in English (Bernhard et al., 2006; Osborn et al., 2007). These two groups of students who struggle with reading face many challenges in school because reading in English is a prerequisite for all of their other subject areas (Hitchcock et al., 2004; Osborn et al., 2007). Once they fall behind one academic year in reading, students who are ELLs and those identified as SLD have a difficult time catching up in later grades (Hitchcock et al.).

In 2005, there were approximately 5.1 million ELLs nationwide (U.S. Department of Education, 2005a). ELLs made up 24.2\% of students enrolled in Miami-Dade County Public Schools during the 2006-2007 school year (Florida School Indicators Report, 
2006-2007). The struggles inherent in learning to read in a second language make ELLs particularly vulnerable to reading challenges in English and multiple grade retentions (Pugh, Sandak, Frost, Moore, \& Mencl, 2005). Even though ELLs are one of the fastest growing groups of students in our schools, and one of the largest groups of learners experiencing reading difficulties (Denton, Anthony, Parker, \& Hasbrouck, 2004), little is known about their reading development in English (Linan-Thompson, Vaughn, HickmanDavis, \& Kouzekanani, 2003).

What has been learned is that ELLs typically find developing reading skills in English particularly challenging and an alarming number of these students are reported as having reading difficulties (Pugh et al., 2005). ELLs have been found to be weak in the areas of academic language, vocabulary, and in the ability to make inferences and analyze text in English (Gersten \& Baker, 2000). After observing the limited number of reading studies on ELLs in relation to their increasing numbers in U.S. schools, Gersten and Baker (2000) concluded that much more research is needed to identify effective reading practices for this population of learners.

Similar to ELLs, students with SLD also find learning to read fluently and with comprehension challenging. Typically, students with SLD are identified by a significant discrepancy between their achievement scores and actual achievement level. Increasingly, however, the definition of SLD is being expanded to include a student's response to individually tailored classroom interventions (Boulineau, Fore, HaganBurke, \& Burke, 2004). These students represent half of the students' nationwide receiving special education services (Case \& Taylor, 2005). Of those identified as having SLD, approximately $80 \%$ have difficulties with reading (Gersten, Fuchs, Williams, \& 
Baker, 2001). Some students with SLD are disfluent readers; meaning that sentences and words are read slowly and laboriously (Pattillo, Heller, Trefry, \& Smith, 2004). Others may read more fluently, but have not developed corresponding reading comprehension skills (Hitchcock et al., 2004). The reading difficulties experienced by students with SLD can lead to reading below grade level, which becomes an increasing problem as these students fall farther behind their peers.

Even more challenged by reading are ELLs identified as having SLD (Tam, Heward, \& Heng, 2006). In addition to challenges in reading, these students often face multiple barriers related to language, culture, disabilities, and mastering content areas (Tam et al., 2006). Approximately 56\% of ELLs with disabilities are diagnosed with SLD (U.S. Department of Education, 2003). ELLs with SLD often exhibit similar characteristics as native English speakers with SLD. They read below grade level, have difficulties with comprehension, and some misbehave or withdraw as a result of reading difficulties (McCardle, Mele-McCarthy, \& Leos, 2005). ELLs should only be identified as having SLD if there is sufficient evidence that the deficiencies are not a result of learning a second language (Gersten \& Woodward, 1994; McCardle et al., 2005).

Research exists that identifies some effective reading strategies for ELLs and students with SLD. ELLs have been shown to benefit from interventions that include vocabulary instruction (Fitzgerald, 1995; Gersten \& Baker, 2000; Tam et al., 2006), repeated exposure to English print (Tam et al., 2006), explicit instruction in comprehension strategies (Denton et al., 2004), and one-on-one instruction with a teacher (Linan-Thompson et al., 2003). Similarly, students with SLD have been found to benefit from interventions that include vocabulary instruction (Therrien \& Kubina, 2006), 
repeated readings (Chard, Vaughn, \& Tayler, 2002; Nelson, Alber, \& Gordy, 2004; Therrien \& Kubina, 2006; Weinstein \& Cooke, 1992), and individual attention (Elbaum et al., 2000). Noting the similarities in effective reading strategies for these two groups of students, Fitzgerald (1995) suggested that ELLs might benefit from interventions known to benefit monolingual students with SLD. These interventions may also have a positive impact on the reading skills of ELLs with SLD.

Research indicates that one intervention that has been shown to be effective in improving reading fluency and comprehension is repeated readings (e.g., Nelson et al., 2004; Tam et al., 2006). Repeated readings is an intervention that targets reading fluency by having the reader repeatedly read a short passage of usually no more than 200 words until their fluency improves (Samuels, 1979; Stahl \& Heubach, 2005; Therrien \& Kubina, 2006). The assumption is that once a student is more fluent in reading the passage, more attention can be focused on comprehension (Samuels, 1979). Repeated readings has been found to be successful with elementary and secondary students (Begeny, Daly, \& Valleley, 2006; Freeland, Skinner, Jackson, McDaniel, \& Smith, 2000), students reading below grade level (Stoddard, Valcante, Sindelar, O'Shea, \& Algozzine, 1993; Tam et al., 2006), students at or above grade level (Bryant et al., 2000), and students with visual impairments (Pattillo et al., 2004). However, literature on the effectiveness of repeated readings on ELLs (Gersten \& Baker, 2000; Tam et al., 2006) and students with SLD (Barley et al., 2002) is limited. Even more limited is research on repeated readings of ELLs with SLD. 


\section{Purpose of the Study}

Although ELLs with SLD are prevalent in many large urban school districts, there is an absence of research of strategies for improving their reading abilities (Tam et al., 2006). Approximately 56\% of ELLs with disabilities are identified as having SLD (U.S. Department of Education, 2003). The population of ELLs with SLD has been projected to grow, particularly in states with high percentages of annual immigration (Case \& Taylor, 2005).

ELLs with SLD struggle to keep up with their English-speaking peers in reading (Denton et al., 2004). Unfortunately, their limited reading fluency and comprehension is a stumbling block in every other subject, as they all employ reading as a means to access information (Rasinski, 2000). Teachers of ELLs with SLD are in need of research to guide their work with these students (Albus, Thurlow, \& Clapper, 2007). The question remains as to what reading strategies can be used to improve reading fluency and comprehension so that these students can succeed in the classroom and beyond.

One approach that shows promise for reading improvement for ELLs with SLD is repeated readings. Yet, limited research examines ELLs with SLD. Prior to this study, only two studies were found that examined the effects of repeated readings on ELLs. One study by Linan-Thompson et al. (2003) included participants who were ELLs and identified as "at risk" of reading disabilities but not identified or diagnosed as having SLD. These researchers found that participants made significant gains in oral reading fluency and passage comprehension when pre- and post-test scores were compared following 58 sessions of supplemental reading instruction that included repeated readings. The other study by Tam et al. (2006) included 5 participants; two were 
identified as ELLs with SLD. However, ELLs with SLD were not specifically targeted for that study. The researchers observed reading improvements following the implementation of a repeated readings intervention that incorporated error correction. However, the authors noted that the findings of their study were preliminary, as there were only 5 participants in the study and the needs of ELLs vary from learner to learner (Tam et al.).

In sum, the study was guided by the increasing numbers of ELLs with SLD in schools with reading challenges and the observed lack of research of effective reading interventions. Further, this study was guided by the positive outcomes observed in repeated readings research on other types of learners and limited research of this strategy with ELLs with SLD. Subsequently, the purpose of this study was to investigate the effects of repeated readings on reading fluency and comprehension of students who are ELLs with SLD.

\section{Statement of the Problem}

This study evaluated the effects of repeated readings on the reading fluency and comprehension of ELLs with SLD. Specifically, it examined the number of words read per minute and the number and type of errors per minute following a single reading of a passage (during baseline probes) and three repeated readings of a passage (during the intervention phase). The study also evaluated differences in the number of correct answers to literal comprehension questions during the baseline probes and the intervention condition. Generalization probes using novel passages at the same level of difficulty determined if any potential gains discovered in the intervention phase were generalized to other passages. Passages from the intervention were used to determine if 
the participants maintained any potential gains 2-, 4-, and 6- weeks after the intervention ended.

Tam et al. (2006) found that two repeated readings interventions resulted in gains in fluency and comprehension in 5 participants, two of whom were ELLs with SLD. During the first intervention that involved reading a passage three times, the oral reading rate of all the participants showed improvements over their performance during baseline. During the second intervention, which involved reading the same passage until meeting a set criterion number of words per minute, 4 of the 5 total participants reached the predetermined fluency criterion of 100 correct words per minute. The mean number of comprehension questions increased from an average of 1 answer correct during baseline. There were an average of 4.1 answers correct during the first intervention and 4.8 answers correct during the second intervention.

This study built on the existing study by Tam et al. (2006) in several ways. First, it specifically targeted ELLs with SLD for the repeated readings intervention. That is, for participants to be eligible for the study, they had to be identified as ELLs with SLD by the school district.

Second, it differed from previous studies in the time and frequency of the intervention sessions. This study consisted of 10 to 20 -minute daily sessions as deemed optimal by Therrien and Kubina (2006) instead of 35-minute sessions 3 times a week (Tam et al., 2006) and 6 minute daily sessions (Nelson et al., 2004). This was done to determine if having a more frequent and intensive intervention such as this study would lead to more rapid gains. Since many ELLs (Fry, 2007) and students with SLD (Osborn 
et al., 2007) lag significantly behind their peers in reading, it was deemed useful to determine if this strategy would lead to rapid gains.

Third, maintenance data were collected and examined. Tam et al. (2006) collected maintenance data that suggested that a higher level of fluency achieved during the intervention conditions might help the participants maintain fluency gains after the interventions ceased. This study yielded information that built on that finding. This study additionally investigated the types of errors made and the number of literal comprehension questions answered correctly during maintenance.

Fourth, generalization data were collected and examined differently than they were in other studies. Tam et al. (2006) conducted generalization probes and found that an increased reading rate on intervention passages may have contributed to positive effects on untaught passages. Weinstein and Cooke (1992) found that fluency increased in unpracticed passages following repeated readings until three observed improvements occurred in intervention. This study extended their research findings by using researcher created passages with $80 \%$ of the same words as passages used during intervention.

Finally, this study was innovative in that it analyzed the types of errors the participants made during reading. No known study has examined the types of errors made by ELLs with SLD during a repeated readings intervention. This information may be used to compare the errors made by these participants to research that reports on the reading error patterns made by native English speakers with SLD (e.g., Barton \& Huynh, 2003). The types of errors made by ELLs with SLD yielded information that no known work has specifically examined. 


\section{Research Questions}

This study examined the effects of a repeated readings intervention on the number of words read aloud per minute, number of errors read aloud per minute, types of errors read aloud per minute, and answers to literal comprehension questions answered aloud by ELLs identified as having SLD who are struggling readers in an urban elementary school. More specifically, the research questions ask, for ELLs with SLD who are struggling readers at an urban elementary school:

1. Will repeated readings result in a change in reading fluency as measured by the number of correct words read aloud per minute?

2. Will repeated readings result in a change in the number and types of reading errors read aloud per minute?

3. Will repeated readings result in a change in the number of literal comprehension questions answered aloud correctly?

4. Will repeated readings result in generalization of the number of (a) correct words read aloud per minute, (b) errors and types of errors read aloud per minute, and (c) literal comprehension questions answered aloud correctly with untaught similar passages?

5. Will repeated readings result in maintenance of the number of (a) correct words read aloud per minute, (b) errors and types of errors read aloud per minute, and (c) literal comprehension questions answered aloud correctly 2-, 4-, and 6- weeks after the intervention? 


\section{Operational Definitions}

In this section, certain terms that are frequently used in this study are defined. Other terms, which are not frequently used but require definitions, are explained as they are introduced.

\section{Addition Error}

An addition error is a word said aloud by the student that is not printed in the passage.

Agreement

Agreement occurs when the researcher and independent raters independently mark the same words and literal comprehension questions as correct or incorrect.

\section{Comprehension}

Comprehension was defined as the number of correct answers to literal comprehension questions pertaining to the reading answered aloud correctly by the participant.

Correct Words per Minute (CWPM)

Correct words per minute (which was also referred to as fluency in this study) was defined as the number of words read aloud correctly per minute of reading (self corrections accepted). A word was counted correct if the student independently pronounced it correctly aloud without prompting within 3 seconds (Nelson et al., 2004).

\section{Disagreement}

Disagreement occurred when the independent rater and researcher did not score the same word or literal comprehension question as correct during independent ratings. 


\section{Disfluent Readers}

Disfluent readers do not read quickly and accurately. Typical characteristics of these readers include reading with poor expression and having difficulty with word recognition, not comprehending what is read, and having little motivation to read (AlberMorgan, Ramp, Anderson, \& Martin, 2007).

English Language Learners (ELLs)

English language learners refer to students learning English and who are less than proficient in English than in their first language (Gersten \& Baker, 2000).

\section{Error Correction}

Error correction is a component of repeated readings (Therrien \& Kubina, 2006). It occurs following a reader error made aloud. It refers to the researcher correctly saying the whole word that was read aloud incorrectly by the participant (Barbetta, Heward, \& Bradley, 1993). The reader is subsequently asked to repeat the word aloud immediately following the error. After the repetition of the word, the entire sentence containing that word is read aloud again by the reader. Once the passage has been read in its entirety, the words that were initially read incorrectly are repeated aloud again by the reader in isolation.

Fluency

The number of words read aloud correctly per minute of reading (Nelson et al., 2004). 


\section{Fluency Assessment}

Fluency, or the number of words read correctly aloud per minute of reading, was measured in a fluency assessment. This assessment involved reading the passage from the beginning for 1 minute.

\section{Generalization}

Generalization is used most often in the applied behavioral analysis literature to indicate behavior changes that occur in nontraining conditions (Stokes \& Baer, 1977). That is, when the effects of behavior-change programs are shown across time, people, settings, and/or related behaviors without direct training in all of those circumstances.

Throughout the study probes were made to test generalization of skills to untaught passages that are similar to those being used in experimental sessions. Generalization passages were approximately 100 words in length (with a minimum of 100 and a maximum of 105 words) and were of the same level as those being used in experimental sessions according to the basal key as well as the Fry Readability Graph (Fry, Fountoukidis, \& Polk, 1985). Additionally, approximately $80 \%$ of the words in the generalization passage came directly from the passages that had been used in the experimental sessions.

\section{Hesitation}

A hesitations is a type of error that refers to a delay of more than 3 seconds from the end of one word read aloud to the beginning of the next word. The researcher counted 3 seconds silently and then stated the correct word to the student so that the student could read on. 
Interobserver Agreement (IOA)

Interobserver agreement is a method for ensuring reliability in the collection of data on a target behavior. It involves two or more independent observers observing the same behavioral episode in order to compare the results.

Literal Comprehension Question

A literal comprehension question is a question that asks the participant to recall something explicitly stated in the text. These questions were used because they demonstrated whether or not the participant had a basic understanding of the passage (Tam et al., 2006).

\section{Literal Comprehension Question Assessment}

The researcher asked five literal comprehension questions one at a time in each condition of the study. The student had 5 seconds to answer each question aloud. Student responses were compared with the answer key provided by the basal reader for that reading passage. Responses matching the answer key that were made within 5 seconds were scored as correct. No response, those not matching the answer key, and/or those made after 5 seconds of silence were scored as incorrect. The total number of correct responses were recorded and graphed.

\section{Maintenance}

Maintenance is the extent to which the learner continues to perform the target behavior after a portion or all of the intervention has been terminated (Cooper, Heron, \& Heward, 2007). 


\section{Mispronunciation}

A mispronunciations is a type of error defined as a printed word that is said aloud incorrectly. For example if the text says "He knows many Latin words" but the student reads aloud "He knows many Latoon words" (Dictionary.com, 2008).

\section{Multiple Baseline Design Across Subjects}

Multiple baseline design across subjects is a single subject research design study model that employs the use of steady state responding. After steady state responding is reached under baseline, the intervention is applied to one participant while the rest continue under baseline. When steady state responding or criterion is reached, the same intervention is applied to next participant and so on (Cooper et al., 2007).

\section{Multiple Probe Baseline Design}

The multiple probe baseline design is a variation of the multiple baseline design. Like the multiple baseline design, interventions are introduced at different times across conditions, participants, or behaviors. The difference is that in a multiple baseline design data are collected continuously during the baseline condition, whereas in the multiple probe design, data are collected intermittently during a baseline probe condition. The intermittent probes are desirable to use in studies such as the present study where collecting continuous baseline data may prove to be reactive, may be impractical or may be unnecessary because a strong assumption of stability can be made prior to the study (Cooper et al., 2007). 


\section{Number of Errors per Minute (EPM)}

This is defined as the total number of errors read aloud during 1 minute of reading. Errors included omissions, additions, mispronunciations, substitutions, and hesitations of more than 3 seconds from one word read to the next (Tam et al., 2006). Omission

An omissions is a type of error defined as a printed word in the passage that is not read aloud by the student.

\section{Repeated Readings}

Repeated readings refer to a method used primarily to build reading fluency. It consists of reading a short passage of no more than 200 words repeatedly aloud until a satisfactory or predetermined level of fluency is reached (Samuels, 1979). Error correction is a component of repeated readings (Therrien \& Kubina, 2006). Error correction strengthens the method by insuring that the participant does not repeatedly practice mistakes.

Specific Learning Disability (SLD)

A specific learning disability (SLD) is a disorder in one or more of the basic psychological processes involved in understanding or in using language, spoken or written, which may manifest itself in the imperfect ability to listen, think, speak, write, spell, or do mathematical calculations (U.S. Department of Education, 2004). SLD is synonymous with the term learning disabilities and is the term currently used.

\section{Struggling Reader}

A struggling reader is a participant reading at least 1 year below grade level in English. 


\section{Substitution}

A substitutions are a type of error defined as stating a word aloud that is different than the printed word. For example, the text says "He saw the park" and the participant reads, "He saw the animal."

Type of Errors per Minute

Throughout the study, the researcher collected data that documented the reading errors that the participants made aloud during oral reading of the passages. The errors were categorized into five types: omissions, additions, mispronunciations, substitutions, and hesitations.

\section{Summary}

Students who read well are able to easily access information both in and beyond school. On the other hand, students who do not read well find assignments challenging and are at risk of facing negative consequences both in and outside of school. ELLs and students with SLD are at risk of having difficulties in reading. ELLs are students who are less fluent in English than in their first language. Students with SLD have a basic psychological processing disorder that affects their ability to use language (U.S. Department of Education, 2004).

ELLs with SLD often find learning to read in English especially challenging (Albus et al., 2007). These students face multiple challenges when learning to read in English that include language, culture, disabilities, and mastering content areas (Tam et al., 2006). ELLs are one of the fastest growing groups of students in U.S. schools (Denton et al., 2004). As the population of ELLs grows, it is reasonable to believe that the population of ELLs with SLD in U.S. schools will also rise. 
While it is known that ELLs with SLD struggle with reading in English, little is known about developing their reading fluency and comprehension (Linan-Thompson et al., 2003). However, research has identified some effective strategies for improving English reading in ELLs and students with SLD as separate groups of learners. These strategies include vocabulary instruction, repeated exposure to text, and individual attention (Fitzgerald, 1995; Gersten \& Baker, 2000; Tam et al., 2006). Since these strategies have been found to be appropriate for students who have been identified as ELLs or as having SLD, this study was undertaken to find if they may be useful in improving the reading fluency and comprehension in students who are ELLs with SLD.

Repeated readings is a method that incorporates some of the strategies suggested for ELLs and students with SLD. Repeated readings involves reading a short passage repeatedly until a satisfactory or predetermined level of fluency is reached (Samuels, 1979). However, there are insufficient data on its success with ELLs (Tam et al., 2006) and students with SLD (Nelson et al., 2004). Only one known study has examined the use of repeated readings with participants who were identified as being ELLs with SLD (i.e., Tam et al.). That study found that the participants had gains in reading fluency and comprehension in each of two repeated readings interventions. However, its findings can only be considered preliminary in the use of repeated readings with this population, as only two of the participants included were ELLs with SLD.

More research is needed to examine the effects of repeated readings on ELLs with SLD. There are increasing numbers of ELLs with SLD in U.S. schools with reading challenges (U.S. Department of Education, 2004). The lack of research on effective reading interventions for this group of students does not address their needs. Research has 
demonstrated positive outcomes when repeated readings is employed with other types of learners (Bryant et al., 2000; Freeland et al., 2000; Pattillo et al., 2004; Stoddard et al., 1993). However, the research on this method and its use with ELLs with SLD is limited (i.e., Tam et al., 2006). Subsequently, this study began to fill a gap in the research base that investigated the effects of repeated readings with students who are identified as ELLs with SLD.

This study used a multiple probe baseline design across subjects to examine the effects of repeated readings on reading fluency, reading errors, and reading comprehension of ELLs with SLD. The research questions addressed the number of correct words read aloud per minute, the number and types of reading errors read aloud per minute, and the number of literal comprehension questions answered aloud correctly. The generalization of the dependent variables on untaught similar passages was examined. Maintenance data were collected 2-, 4-, and 6-weeks after the intervention concluded. 


\section{CHAPTER II}

\section{LITERATURE REVIEW}

Reading is a skill that allows access to information and opportunity. Some students, particularly English language learners (ELLs) with specific learning disabilities (SLD), find learning to read in English challenging (Tam et al., 2006). This study used repeated readings as an intervention to target reading fluency and comprehension in ELLs with SLD.

This chapter provides a review of the literature related to this research study. A general discussion of the characteristics of students who do and do not read well is presented initially. This is followed by the reading performances in English of ELLs, students with SLD, and ELLs with SLD. Next, a discussion of effective reading strategies for these two groups of struggling readers is provided. Finally, there is a thorough presentation of the research on repeated readings, the reading intervention investigated in this study.

\section{Characteristics of Students Who Do and Do Not Read Well}

Students who read well are those who read fluently and with comprehension (Rasinski, 2000). These readers can summarize, interpret, and accept or reject the information on the printed page (Pressley et al., 2006). According to Valencia and Pearson (1986), students who read well use prior knowledge to help construct meaning from the text and to draw inferences at the lexical, syntactic, and textual levels. Furthermore, effective readers provide appropriate responses to questions about text. They are able to employ various reading strategies to help them understand what they 
read, to read long passages with ease and, to complete assignments in a reasonable time (Rasinski, 2000). These readers tend to feel good about their ability to read and are selfmotivated enough to want to read more (Rasinski, 2000). Because they can read well, they are expected to attain social and economic success beyond the school years (Snow et al., 1998).

Students who do not read well, conversely, find it challenging to learn how to read fluently and with comprehension. These students read laboriously and slowly and dedicate a great deal of energy to decoding the letters on the page, with little attention to comprehension (Chard, Vaughn, \& Tyler, 2002; Pressley et al., 2006). Unfortunately, these readers are associated with academic failure (Hitchcock et al., 2004). They face numerous poor academic and social outcomes because of their lack of adequate literacy skills (Hitchcock et al.). In the classroom, these students cannot perform at the level of their peers. They often are exposed to less information because of their inability to read well (Hitchcock et al.). Their frustrations often lead to exhibitions of disruptive or withdrawn behavior in the classroom to avoid reading-related activities (Hitchcock et al.). Potential poor social outcomes for students who do not read well include limited employment opportunities and a greater likelihood of living in poverty (Elbaum et al., 2000; Hitchcock et al.).

Students significantly at risk of not reading well and experiencing the associated negative outcomes are ELLs (Bernhard et al., 2006) and those with SLD (Osborn et al., 2007). Because being able to read well and comprehend material is necessary for success in all of the subject areas, these two groups of students face many school challenges 
(Hitchcock et al., 2004; Osborn et al.). Once they fall behind in reading, ELLs and students with SLD have a difficult time catching up in later grades (Hitchcock et al.).

English Language Learners and Reading

ELLs are students who have been identified as being less than proficient in English than their first language (Gersten \& Baker, 2000). This term encompasses students who may communicate effectively in social situations, but struggle with academic English (Gersten \& Baker, 2000). In Miami-Dade County Public Schools (MDCPS) students who are exposed to a language other than English are administered the Oral Language Proficiency Scale (Dade County Board of Public Instruction, 1978). The data gathered from this assessment are reported in the form of an ESOL Level ranging from I-V, whereas I represents highly limited English language proficiency and V equals fluency in English (Miami Dade County Public Schools, 2005).

Over the last several decades, the population of ELLs in the U.S. has increased every year (Case \& Taylor, 2005). For example, from 1991 to 2003, this population increased by 105\% (U.S. Department of Education, 2003). By 2015, it is estimated that $30 \%$ of the nation's school population will be the children of immigrants who speak languages other than English (U.S. Department of Education, 2003). With such a remarkable increase estimated over a short period of time, their needs to be more research examining ELLs and their acquisition of English reading skills (Gersten \& Baker, 2000).

ELLs are one of the largest groups of learners experiencing English reading difficulties in U.S. schools (Denton et al., 2004). The National Assessment for Educational Progress (NAEP) found that nearly three-quarters of fourth-grade ELLs scored at the lowest level possible for reading in national testing in 2005 (Fry, 2007). 
This report found that ELLs nationally tended to be concentrated in the lower achievement levels in English reading and trail their White, native English speaking peers by a gap of 47 percentage points (Fry, 2007). Nationally, ELLs trail further behind their peers in reading than in other subject areas.

Because ELLs have not mastered the English language, they typically find it challenging to acquire reading skills in English (Pugh et al., 2005). Obtaining reading fluency and accuracy is therefore a daunting task (Brisk \& Herrington, 2000). To become a better reader, amongst other skills, ELLs need to acquire knowledge of English idiosyncrasies (Brisk \& Herrington, 2000) such as the multiple meanings of certain words and the various sounds a single letter combination can make (Linan-Thompson et al., 2003). Also, these students must internalize the sounds, rhythms and patterns of the English language, something that a native reader knows prior to entering school (Brisk \& Herrington, 2000).

Contextual and personal variables make it difficult to list the general characteristics of ELLs who are struggling with reading (Brisk \& Herrington, 2000). ELLs come from various backgrounds and have varied experiences. Some have had schooling in their native language and have acquired reading skills that need to be transferred to English (Pugh et al., 2005). Some have not had consistent schooling and do not read well in any language. Some ELLs have a background in a language that resembles English in word structure, word order, or sound, while others do not. These differences in their schooling and reading backgrounds mean that the reading abilities of ELLs need to be reviewed individually, and each may face unique challenges in learning how to read in English. However, all ELLs have a large amount of new information to internalize. Therefore, 
most ELLs have a slower rate of acquiring reading skills in English than their monolingual peers (Pugh et al.). Although, little is known about the English reading development of ELLs (Linan-Thompson et al., 2003), it is known that it can take more than 7 years for these students to become fluent speakers and readers in a second language (Krashen \& Terrell, 1983).

There have been two research reviews examining reading development in ELLs. Gersten and Baker (2000) used a rigorous qualitative method, a multivocal synthesis, to evaluate the results of several intervention and descriptive studies. They found several specific instructional variables to be effective in working with ELLs such as vocabulary instruction and cooperative learning strategies. Fitzgerald (1995) found that ELLs use cognitive processes that are similar to those of native-language readers. Specifically, their vocabulary knowledge plays an important role in reading and comprehension. They monitor their own reading and recognize problems to self-correct. Further, Fitzgerald found that these students use schema and prior knowledge and understand passages that are more congruent with their experiences better than those that are not. However, while the same cognitive processes as native English speakers are used, some facets of those processes may be used more slowly in ELLs. Fitzgerald postulated that the same reading interventions used with native speakers who struggle with reading might therefore be successful with ELLs.

Students with Specific Learning Disabilities and Reading Many students with SLD also find it challenging to learn to read fluently and with comprehension. The Individuals with Disabilities Education Act (U.S. Department of Education, 2004) defines SLD as a disorder in one or more of the basic psychological 
processes involved in understanding or in using language, spoken or written, which may manifest itself in the imperfect ability to listen, think, speak, write, spell, or do mathematical calculations (U.S. Department of Education, 2004). Half of the student's nationwide receiving special education services are students who are labeled as having SLD (Case \& Taylor, 2005). Of those identified as having SLD, approximately $80 \%$ have difficulties with reading (Gersten et al., 2001). Typically, reading ability is the reason for the initial referral of these students to special education.

Teachers of students with SLD who struggle with reading report that they tend to function below their peers in nearly every subject area (Osborn et al., 2007). Some readers with SLD struggle with basic, automatic word identification and decoding and are subsequently disfluent, meaning that sentences and words are read slowly and laboriously (Manset-Williamson \& Nelson, 2005; Pattillo et al., 2004). Chard et al. (2002) noted that students with SLD are most at risk for having poor fluency because too much effort is spent on decoding and not enough effort is spent on higher-level processes such as comprehension.

Students with SLD, who struggle with reading, cannot read well enough to complete assignments in school (Hitchcock et al., 2004; Nathan \& Stanovich, 1991). Because they read fewer words per minute, these students need to devote a greater amount of time and energy to their assignments than their peers (Rasinski, 2000). This may lead to frustration. Frustration may lead to these students reading less and choosing not to read when other activity options are available to them (Rasinski, 2000). Choosing not to read contributes to the lack of reading practice, which further widens the gap between these students with SLD and their peers. 
Chard et al., (2002) conducted a synthesis of research studies on students with SLD. The results suggested that effective interventions include explicit models of reading fluency, multiple opportunities to read familiar text repeatedly, and the opportunity to have some form of corrective feedback. The focus of only one study was reading comprehension however, most of the studies indicated that there was an increase in comprehension when participants improved their fluency.

Therrien and Kubina (2006) summarized similar points. They stated that in order for students with SLD to improve their reading fluency and comprehension, they should repeatedly read passages with a competent tutor and receive corrective feedback (Therrien \& Kubina, 2006). Additionally they made reference to the time commitment required in implementing a repeated reading intervention. Their research indicated that in order to see maximum gains in reading the intervention should last 10 to 20 minutes per session and should occur between 3 to 5 times per week (Therrien \& Kubina, 2006).

English Language Learners with Specific Learning Disabilities and Reading Students who are at the intersection of these two groups, ELLs with SLD, face multiple challenges including language, culture, and disabilities (Tam et al., 2006). These students are developing a bilingual brain while dealing with processing issues that make learning to read fluently and with comprehension difficult without appropriate supports (Pugh et al., 2005). Further, it is not always clear whether or not the reading difficulties are related to the acquisition of English or to SLD.

The National Office of English Language Acquisition estimated that $9 \%$ of all ELLs in the U.S. in kindergarten through $12^{\text {th }}$-grade were identified as having disabilities during the 2001-2002 school year (U.S. Department of Education, 2003). Of those ELLs 
identified as having disabilities, 56\% were identified as having SLD (U.S. Department of Education, 2003). According to the National Assessment of Educational Progress being an ELL dramatically increases the probability of a student having reading challenges (Fry, 2007). Students who are both ELLs and who have SLD require more attention to assist them in reaching the levels of English reading fluency and comprehension of their native English speaking peers without disabilities (Albus et al., 2007; Fry, 2007).

\section{Repeated Readings}

Repeated readings is a reading intervention that targets fluency and comprehension (Nelson et al., 2004; Tam et al., 2006; Therrien et al., 2006). It involves having the reader repeatedly read a short passage of usually no more than 200 words repeatedly until a satisfactory or predetermined level of fluency is reached (Samuels, 1979; Stahl \& Heubach, 2005; Therrien \& Kubina, 2006). Fluency refers to the reader's ability to read quickly and accurately (Manset-Williamson \& Nelson, 2005). Reading fluency is generally measured by counting the number of words read correctly per minute Repeated readings grew out of the Automaticity Theory (LaBerge \& Samuels, 1974; Samuels \& Flor, 1997) which stated that a fluent reader could decode text without devoting attention to the process of reading, therefore leaving attention free to dedicate to comprehension of the text (Samuels, 1979). According to this theory, beginning readers need to focus on letters and sounds, but as they become more fluent readers they can focus their attention on phrases, sentences, and meaning. Samuels (1979) stated that the practice provided in repeated readings, makes the decoding necessary for reading automatic, therefore leading to better comprehension. 
While there has been a substantial amount of research on repeated readings with students of varying ages and abilities (Bryant et al., 2000; Freeland et al., 2000; Pattillo et al., 2004; Stoddard et al., 1993), there exists only limited research regarding its effectiveness with ELLs (Gersten \& Baker, 2000; Tam et al., 2006) and students with SLD (Barley et al., 2002). A thorough search of the literature revealed only two studies conducted with ELLs (Linan-Thompson et al., 2003; Tam et al., 2006). A somewhat broader base of research exists examining repeated readings with students who have SLD (Begeny et al., 2006; Nelson et al., 2004; Therrien et al., 2006; Weinstein \& Cooke, 1992). Further, only one study was found that included 2 participants who were identified as ELLs with SLD (Tam et al.).

Following is a review of the two studies examining the effects of repeated readings on ELLs. One of these studies (Tam et al., 2006) included 2 participants who were identified as ELLs with SLD. This is followed by a review of four studies examining the effects of repeated readings interventions on students with SLD. Finally, the lack of known studies that examine repeated readings with ELLs with SLD is discussed.

\section{English Language Learners and Repeated Readings}

Fitzgerald (1995) concluded that teachers of ELLs should use sound principles of reading instruction based on current research with native speakers as well as with ELLs. Since repeated reading has been examined with native language speakers who are struggling with reading (e.g., Chard et al., 2002; Nelson et al., 2004) and has been found to be effective, it seems logical that its effects on ELLs should be investigated. The limited studies examining this method with ELLs, however, indicate a need to broaden the research base in this area. 
Linan-Thompson et al. (2003) conducted a statistical analysis of pre- and post- test scores with ELLs who participated in an intervention that included repeated readings. The participants were 26 ELLs in the second grade who were identified by their teachers as being at risk for reading difficulties. The intervention included repeated readings and other reading strategies. Specifically, the intervention was 30 minutes long and included (a) 5 minutes of repeated readings, (b) 5 minutes of phonemic awareness instruction, (c) 10 minutes of instructional level reading, (d) 5 minutes of word study, and (e) 2 to 3 minutes of writing. All the participants made statistically significant gains in oral reading fluency. All but three students made more than 6 months worth of gains during the 3month intervention. The most dramatic gains were made in reading fluency (LinanThompson et al., 2003). The gains in fluency indicated that repeated readings was valuable to the participants, however, the multi-componential nature of the intervention made it difficult to determine whether the repeated readings alone accounted for the gains.

In a study specifically targeting ELLs struggling with reading, Tam et al. (2006) also found an increase in oral reading fluency following a repeated readings intervention. They used a multiple baseline across subjects design to evaluate the effects of a repeated reading intervention program on oral reading rate and comprehension. The participants were five elementary aged ELLs reading below grade level; two of them were diagnosed with SLD. The first intervention condition consisted of asking the participants to read the passage as fast as they could three times. The second intervention condition was identical except that the same passage was used until the learner reached a predetermined number of words read correctly per minute. During the first condition, the oral reading rate of all 
5 participants showed improvements over their performance during baseline. During the second condition, 4 of the 5 participants reached the predetermined fluency criterion of 100 correct words per minute. The mean number of comprehension questions answered correctly per session was notably higher during both intervention conditions than during baseline. Specifically, there was an average of one answer correct during baseline and 4.1 and 4.8 answers correct during the first and second intervention conditions respectively.

In sum, the research in repeated readings is preliminary but demonstrates promise for ELLs. Linan-Thompson et al. (2003) and Tam et al. (2006) both found an increase in oral reading fluency in ELLs following the implementation of interventions that included repeated readings. Tam et al. also found that participants answered more literal comprehension questions correctly following the repeated readings interventions. However, the fact that there are only two studies on repeated readings and ELLs demonstrates that this research is limited, and that less is known about the effectiveness of this approach with ELLs than of their native English speaking peers.

Students with Specific Learning Disabilities and Repeated Readings A somewhat broader base of empirical studies exists that examines repeated readings and students with SLD. Recently, research in this area has focused on different aspects of the repeated readings process. One aspect is comparing the results of repeatedly reading a short passage until a criterion reading rate is reached to repeatedly reading a set number of times or until consecutive improvements are made (Tam et al., 2006; Weinstein \& Cooke, 1992). Another researched aspect is the pairing of repeated readings with some form of error correction (Begeny et al., 2006; Nelson, et al., 2004; Therrien et al., 2006). Generally, it is agreed that students should be asked to read 
passages that will allow them to reach criterion in a reasonable amount of time, and that error correction should be a natural part of repeated readings so that errors are not practiced repeatedly (Therrien \& Kubina, 2006).

In a classic study, Weinstein and Cooke (1992) examined the effects of two repeated readings interventions on generalization of fluency. The participants were four beginning readers in the second and third grades with SLD. They used a single subject ABACA design in which two interventions were examined. In both interventions, the participants listened to an audiotape of a passage read at a rate of 100 words per minute. Then, they were asked to read the passage as quickly and well as they could. The first intervention was a fixed criterion intervention, in which the participant had to continue reading until reaching a 90 word per minute criterion. The second intervention was called the improvements criterion. It involved reading the same passage until three consecutive improvements were recorded. The researchers recorded if an increase the fluency rate (as measured by the number of correct words read per minute) resulted following each of the two interventions.

All 4 participants experienced gains in their mean number of words read per minute in both interventions as compared to baseline. However, in the fixed criterion intervention, the students read an average of $62 \%$ more words per minute and in the improvements criterion intervention the students read 52\% more words per minute than in baseline. Generalization data favored the improvements criterion intervention. The number of correct words per minute in unpracticed stories increased from baseline following the improvements phase. Correct words per minute decreased from the preceding baseline on unpracticed stories following the criterion phase. While the study 
was somewhat limited by the small number of participants (4) and time constraints (some intervention phases had to be truncated before the participants reached criterion due to the school schedule), the researchers demonstrated that both repeated readings interventions proved to be an effective tool in assisting all four students in making gains in fluency (Weinstein \& Cooke, 1992).

More recently, Nelson et al. (2004) found gains in fluency when repeated readings was implemented with 4 second grade participants with reading difficulties. Three of the 4 participants were identified as having SLD. A multiple-baseline design was used to examine the effects of two interventions on reading accuracy and fluency: systematic error correction and systematic error correction paired with repeated readings. In the systematic error correction phase, participants received feedback for each misread word during the oral reading of a passage. Then the participant practiced each misread word in isolation following the reading. During the error correction paired with repeated readings phase, the error correction procedures mentioned above were followed by three oneminute timed readings of the passage.

The results of Nelson et al. (2004) demonstrated that with systematic error correction there were fewer reading errors per minute for all 4 participants. However, there was only a minimal increase in the number of words read correctly per minute. Conversely, when repeated readings was paired with systematic error correction all four participants improved in both reading accuracy and fluency. In this phase, all 4 participants read correctly from 12.6 to 14.5 words more per minute than in the systematic error correction phase. They also had similar or lower error rates during the repeated readings paired with error correction phase as compared to the systematic error 
correction phase. Maintenance data with previously read material demonstrated that the number of words read per minute increased slightly from baseline for three of the participants.

Begeny et al., (2006) also compared the effects of repeated readings and an error correction method. Specifically, the authors employed an alternating treatments design to determine the effects of four different reading treatments on an 8-year old boy with SLD. The first treatment, baseline, consisted of a single reading of a passage to assess reading fluency. In the second treatment, phrase drill error correction, the instructor modeled a word after it was read incorrectly and prompted the student to repeat the phrase that contained the misread word. This was followed by a fluency assessment. In the third treatment, entitled repeated readings, the participant read a passage twice before being assessed for fluency. In the final treatment, the reward treatment, the participant read a previously administered passage followed by a fluency assessment and then was rewarded with a preferred reward if the passage was read faster and with the same or fewer errors than the previous time the passage was administered.

The results of Begeny et al. (2006) indicated that the repeated readings and phrase drill treatments resulted in reading gains. During the baseline treatment the mean correct words read per minute was 36.13. During the repeated readings treatment, there was an average of 59.63 correct words per minute. The phrase drill treatment had an average of 60 correct words per minute. The results indicated that repeated readings improved oral reading fluency with an overall increase of 23.5 words read correctly per minute and an overall decrease of 1.62 reading errors per minute as compared to baseline. Also, it preliminarily demonstrated that a critical component of repeated readings was the use of 
an error correction procedure that included the instructor modeling the correct pronunciation of the word and the student imitating the model. The importance of error correction was demonstrated by a decrease of three reading errors per minute during the phrase drill error correction treatment as compared to the baseline treatment.

Similarly, Therrien et al. (2006) incorporated error correction methods in a repeated readings study. They used a pre/post examination model study to ascertain if repeated readings combined with a question generation component was effective at improving the reading fluency and comprehension of fourth- to eighth-grade participants with SLD or who were struggling readers. Participants were randomly assigned to a treatment or control group. During treatment, each participant read a passage until a criterion number of correct words per minute was attained or until the passage was read four times. The participant was given corrective feedback when a reading error was made aloud. The participant was then prompted to read and answer questions from a cue card pertaining to the passage. The entire intervention series was repeated again in the following session with new material adjusted by the researcher depending on the participant's ability to reach the criterion. The control group did not receive the treatment. Instead, the control group received reading instruction in the classroom while the treatment group received the treatment in addition to the reading instruction in the classroom.

The results of the 4-month study demonstrated that participants receiving the intervention made significant gains in reading fluency and comprehension. From pre- to post-test they improved their reading fluency by an average of 13 correct words per minute while the control group improved reading fluency by an average of only 2.28 
correct words per minute. The treatment group also improved their ability to answer inferential comprehension questions by an average of two more questions correct on passages that were reread as compared to passages that were read only once.

As a whole, the research in the use of repeated readings and students with SLD has resulted in positive outcomes. Weinstein and Cooke (1992) saw increases in reading fluency in students with SLD. The study by Nelson et al. (2004) demonstrated that the combined use of repeated readings with error correction resulted in increases in reading fluency and decreases in error rates as compared to baselines in which a passage was read once. Begeny et al. (2006) found that significant gains were made by a student who received an intervention that incorporated repeated readings and corrective feedback. Therrien et al. (2006) found that struggling readers and students with SLD receiving repeated readings interventions made increases in oral reading fluency as well as gains in the number of correctly answered questions about the passages read. These results are encouraging as these data can be used to support the use and further examination of repeated readings as an intervention for students with SLD who struggle with reading. English Language Learners with Specific Learning Disabilities and Repeated Readings No known studies to date have specifically targeted ELLs with SLD to examine the effects of repeated readings as compared to a single reading of a passage. However, one study mentioned previously conducted by Tam et al. (2006) did include 2 participants who were identified as ELLs with SLD. The researchers found that the participants had an increase in fluency and comprehension in each of two repeated readings interventions. One intervention involved reading a passage until a criterion of 100 words per minute was reached. The other treatment involved reading a passage until three consecutive 
increases in words per minute were recorded. One of the two ELLs with SLD was initially identified as a struggling reader reading 2 years below grade level. Through the course of the study, however, it was found that she could read material that was at a higher level. The researchers noted that she read material at her grade level for the remainder of the study following the discovery that she could indeed read better than was initially assessed (Tam et al.). The student's ability to read passages on grade level led the researchers to question whether this student was truly an ELL with SLD who was struggling with reading. The article does not indicate whether or not the student was reevaluated. Therefore, although the study had two students who were identified as ELLs with SLD, there may have only been one who met this criterion.

Preliminarily, the results of Tam et al. (2006) suggest that repeated readings could be considered as a reading strategy for students who are ELLs with SLD. However, much more research is needed to raise the level of confidence in this approach as an evidencebased intervention for ELLs with SLD.

\section{Summary}

Reading is a skill that allows access to opportunity. There are several positive outcomes associated with being able to read well and several negative outcomes associated with not being able to read well. Positive outcomes include social and economic success beyond the school years (Snow et al., 1998). Negative outcomes include limited employment opportunities and a greater likelihood of living in poverty (Elbaum et al., 2000; Hitchcock et al., 2004).

ELLs (Bernhard et al., 2006) and students with SLD (Osborn et al., 2007) are at particularly high risk of facing the negative outcomes of not reading well. ELLs are 
students who have been identified as being less than proficient in English than their first language (Gersten \& Baker, 2000). Students with SLD have a disorder in one or more of the basic psychological processes involved in understanding or in using language (U.S. Department of Education, 2004). Students who are ELLs with SLD face multiple challenges in acquiring reading skills in English and are neglected in the literature on reading interventions. The strategies that have been shown to be effective in assisting students who are ELLs or who have SLD are similar and may be successful with ELLs with SLD (Fitzgerald, 1995).

The method of repeated readings, which came out of the Automaticity Theory of LaBerge and Samuels (1974), is used to improve reading fluency and comprehension in struggling readers. Repeated readings involves having the reader repeatedly read a short passage of usually no more than two hundred words repeatedly until a satisfactory or predetermined level of fluency is reached (Samuels, 1979; Stahl \& Heubach, 2005; Therrien \& Kubina, 2006). While there has been a considerable research on repeated readings in general, research with ELLs (Gersten \& Baker, 2000; Tam et al., 2006) and students with SLD (Barley et al., 2002) is limited. Even more limited is the research examining the method of repeated readings with students who are ELLs with SLD (Tam et al.).

Only a small body of literature has demonstrated the effectiveness of repeated readings with ELLs (Linan-Thompson et al., 2003; Tam et al., 2006). Linan-Thompson et al. conducted a statistical analysis of pre- and post- test scores with ELLs who participated in an intervention that included repeated readings. The most dramatic gains were made in reading fluency. The gains in fluency indicated that repeated readings was 
valuable to the participants, however, the multi-component nature of the intervention made it difficult to determine whether the repeated readings alone accounted for the gains. Tam et al. also found an increase in oral reading fluency following a repeated readings intervention. During the second condition, 4 of 5 participants reached the predetermined fluency criterion of 100 correct words per minute. The mean number of comprehension questions answered correctly per session was notably higher during both intervention conditions than during baseline.

A slightly larger body of literature exists that examines repeated readings with students who with SLD. Chard et al. (2002) conducted a synthesis of research studies on students with SLD and found that that effective interventions include explicit models of reading fluency, multiple opportunities to read familiar text repeatedly, and the opportunity to have corrective feedback. Therrien and Kubina (2006) echoed this when stating that students with SLD should repeatedly read passages with a competent tutor and receive corrective feedback. Weinstein and Cooke (1992) and Nelson et al. (2004) demonstrated that repeated readings interventions proved to be an effective tool in assisting participants with SLD in making gains in fluency. Repeated readings improved oral reading fluency with an overall increase of 23.5 words read correctly per minute and an overall decrease of 1.62 reading errors per minute as compared to baseline in a single subject study by Begeny et al. (2006). Therrien and Kubina (2006) ascertained that a repeated reading intervention, which included a question generation component, was effective at improving the reading fluency and comprehension in fourth- to eighth-grade participants with SLD or who were struggling readers. Taken together, repeated readings has been shown to be an effective tool in improving reading in students with SLD. 
This review clearly demonstrates the need for additional research on the effects of repeated readings on students who are ELLs with SLD as only one study included two students who meet this criterion (Tam et al., 2006). Although this population is steadily increasing in public schools throughout the country (U.S. Department of Education, 2005a), there is a dearth of information to guide educators in assisting ELLs with SLD in reading acquisition (Tam et al.). There is a need use systematic approaches to determine effective reading instructional techniques for ELLs (Gersten \& Baker, 2000; LinanThompson et al., 2003). Repeated readings may be an effective intervention for improving English reading in ELLs with SLD.

This study examined the effects of repeated readings on English reading fluency, accuracy and comprehension of ELLs with SLD who are struggling readers. This was accomplished by examining the effects of a repeated readings intervention on the number of words read correctly aloud per minute, the number of errors read aloud per minute, the types of errors read aloud per minute, and the ability to correctly answer literal comprehension questions aloud. Also, the study measured generalization and maintenance of the same reading skills. 


\section{CHAPTER III}

\section{METHOD}

This study examined the effects of repeated readings on reading fluency, reading errors, and reading comprehension of third-, fourth-, and fifth-grade English language learners (ELLs) with specific learning disabilities (SLD) who are struggling readers. Also examined were the maintenance and generalization of the skills acquired throughout the course of the intervention.

This chapter presents information about the study's participants, setting, and materials. The dependent variables are identified and explained. This is followed by a description of the experimental design used in this study. The general procedures section details the steps taken prior to and during the course of the study. This chapter concludes with a summary.

\section{Participants}

Participants were 4 ELLs with SLD who were struggling readers in the third-, fourth-, and fifth-grade who were nominated by their teachers for participation in this study (see Table 1, page 43). Participant names used throughout the study are pseudonyms. The nominating teachers were special, general, and English for Speakers of Other Languages (ESOL) teachers who had known the participants for at least 1 year. Teacher nominations were based on their experiences with the participants and the participants' performances in reading tasks in their classes. The teacher nominations were used as part of the selection procedures as teachers are an invaluable source of information regarding their students (Abidin \& Robinson, 2002), and teacher nomination 
is considered an ideal method for finding the participants most in need for intervention by single subject researchers (Cooper et al., 2007; Tam et al., 2006). In addition, written parental permission (see Appendix A) and a signed participant assent form were required for each participant to take part in the study (see Appendix B).

The participants functioned at ESOL Levels III, IV, or V. Participants functioning at Levels I and II were not considered for this study because they are not at a proficiency level in English in which it is appropriate to assess reading ability in English (Krashen \& Terrell, 1983). Miami-Dade County Public Schools (M-DCPS) initially identifies ELLs by administering a three question Home Language Survey to parents on the day of registration (see Appendix C). An answer of "yes" on any questions in the survey indicates that the student is exposed to a language other than English. At this point, permission is requested to administer the Oral Language Proficiency Scale (OLPS; Dade County Board of Public Instruction, 1978) or another age appropriate language assessment. Data from this assessment then yields the school with an ESOL Level ranging from I-V where I represents highly limited English language proficiency and V equals fluency in English (M-DCPS, 2005).

Also, the M-DCPS district identified the participants as having SLD.

Preliminarily, participants with SLD in M-DCPS are identified through teacher, parent, or specialist recommendation. Then, the district's evaluation procedures provide for the use of valid tests and evaluation materials, administered and interpreted by trained personnel, in conformance with instructions provided by the producer of the materials. For a student to meet the eligibility criterion for SLD the following must be documented: (a) general education strategies must have been tried and found to be ineffective, (b) there must be a 
disorder in one or more basic psychological processes, (c) there must be a discrepancy of one standard deviation or more between an intellectual standard score and achievement standard score, and (d) there must be learning problems that are not due primarily to other handicapping conditions (Miami-Dade County Public Schools, 2007).

In addition, participants were identified as struggling readers. In this study, struggling readers were defined as reading at least 1 year below grade level in English (Nelson et al., 2004), based on grade level assessments given at the beginning and midpoint of the school year by the classroom teacher. At the target school, these assessments were the Diagnostic Reading Assessments (Roswell, Chall, Curtis, \& Kearns, 2005), the Dynamic Indicators of Basic Early Literacy Skills (Good \& Kaminski, 2002), and the Woodcock-Johnson III Diagnostic Reading Battery (Woodcock, Mather, Schrank, 2001). In addition, the Analytical Reading Inventory (ARI; Woods \& Moe, 2006), an informal assessment, was given by the researcher prior to the start of the study to confirm that the participant's reading level was at least 1 year below their grade level. The ARI also determined the participants' instructional reading level. A description of each participant and a summary table of characteristics in Table 1 (page 43) follows. Participant 1

Tano (pseudonym) was a 10-year-old Hispanic boy, who had been retained in the third grade because he had not passed the Florida Comprehensive Assessment Test (FCAT). He functioned at ESOL level III and he reported speaking Spanish at home. He met criteria for an Individualized Education Plan (IEP) because of a specific learning disability and his ARI results indicated that his instructional reading level was second grade. Tano accessed the curriculum in a general-education classroom with no pull out 
services. His class was consisted of 12 retained third graders, eight of whom were on IEPs for varying exceptionalities. Two certified teachers, one general elementary education and the other special education, taught the class. During the course of the study, the class received periodic group instruction from an intern who was completing her associate teaching credits for a nearby university.

Table 1

Demographic and Analytical Reading Inventory Data

\begin{tabular}{lccccccc}
\hline Participant* & Gender & Age & Grade & Ethnicity & $\begin{array}{c}\text { ESOL } \\
\text { Level }\end{array}$ & Disability & $\begin{array}{c}\text { ARI } \\
\text { Instructional } \\
\text { Reading } \\
\text { Level }\end{array}$ \\
\hline Tano & M & 10 & $3^{* *}$ & Hispanic & III & SLD & 2 \\
Luci & F & 11 & 4 & Hispanic & IV & SLD & 2 \\
Sandra & F & 10 & $3^{* *}$ & Hispanic & III & SLD & 1 \\
Gema & F & 11 & 5 & Hispanic & III & SLD & 1 \\
\hline $\begin{array}{l}\text { Note. *All participant names are pseudonyms. } \\
\text { **Indicates a participant who is repeating the grade because of failure to pass the Florida Comprehensive Assessment Test }\end{array}$ \\
(FCAT).
\end{tabular}

\section{Participant 2}

Luci (pseudonym) was an 11-year-old Hispanic girl in the fourth grade. She functioned at ESOL level IV and reported speaking Spanish at home. She met criteria for an Individualized Education Plan (IEP) due to an SLD, and her ARI results indicated her instructional reading level was second grade. Luci accessed the curriculum in a general education class with no pull out services. Her class consisted of 10 general education students and 13 exceptional education students who were on IEPs for varying 
exceptionalities. Two certified teachers, one general elementary education and the other special education, taught the class.

\section{Participant 3}

Sandra (pseudonym) was a 10-year-old Hispanic girl, who had been retained in the third grade because she did not pass the Florida Comprehensive Assessment Test (FCAT). She functioned at ESOL level III and reported speaking Spanish at home. She met criteria for an Individualized Education Plan (IEP) due to a SLD and her ARI results indicated that her instructional reading level was first grade. Sandra accessed the curriculum in a general-education classroom along with pull out services. She was educated in a resource room for reading instruction for $1 \frac{1}{2}$ hours daily. Her class was made up of 12 retained third graders, eight of whom were on IEPs for varying exceptionalities. Two certified teachers, one general elementary education and the other special education, taught the class. During the course of the study, the class also received periodic group instruction from an intern.

\section{Participant 4}

Gema (pseudonym) was an 11-year-old Hispanic girl in the fifth grade. She functioned at ESOL level III and reported speaking Spanish at home. She met criteria for an Individualized Education Plan (IEP) due to a SLD and her ARI results indicated that her instructional reading level was first grade. Gema accessed the curriculum in a general education classroom with no pull out services. Her class consisted of 12 general education students and 12 exceptional education students were on IEPs for varying exceptionalities. The students rotated as a group between four certified general education teachers who each taught a specific subject area. Additionally, a certified special 
education teacher rotated with the students in order to offer assistance. Gema participated in the study during her homeroom class, as her teachers and parents expressed concern that she would miss instruction in her subject area classes.

\section{Setting}

This study took place at an urban public elementary school in the M-DCPS district. Currently, 38.5\%, of the school's 1,036 students are identified as ELLs (MDCPS, 2006). The researcher conducted all one-to-one experimental sessions in a quiet room in the media center with no one else in the room and with desks that allowed the researcher and participant to sit next to each other. The participants were pulled out of their general education classroom for approximately 10 to 20 minutes in order to participate in the study. The experimental sessions occurred in the morning (9 to $11 \mathrm{AM}$ ) and afternoon (1 to 3 PM). Each student attended one session daily (5 days a week) at approximately the same time each day for approximately 12 weeks.

\section{Materials}

Following is a description of the study's materials. The materials list includes several forms (consent, assent, treatment fidelity, IOA, data collection), an informal reading inventory, a readability graph, reading passages, and literal reading comprehension questions. Additionally, a digital recording device was used to make audio recordings of each session including generalization and maintenance testing sessions.

\section{Parental Consent Form}

Parents were provided with a consent form written in both English and Spanish for their child's participation in the research study (see Appendix A). The form provided 
a brief description of the study, information regarding the study's duration, and the expectations for parental and student commitments. Researcher and university contact information were provided on the form.

\section{Participant Assent Form}

Students whose parents signed the consent form were read the participant's assent to participate in the research study form (see Appendix B). The form provided a brief description of the study, information regarding the study's duration, and the expectations for the researcher and the student. It was written free of jargon and using developmentally appropriate language for the potential participant. The researcher read this form to the participant and answered any questions the participant had. Researcher and university contact information was provided on the form. The participant was given a copy of the signed form.

\section{Treatment Fidelity Checklist}

A treatment fidelity checklist that included the procedures for each phase in the study was used during each session by the researcher (see Appendix D). The independent rater verified that the researcher is implemented proper procedure by independently filling out a treatment fidelity form for $25 \%$ of sessions.

\section{Interobserver Agreement (IOA) Forms}

Three independent raters completed data collection forms for approximately $25 \%$ of the study's sessions (see Appendix F). An independent rater completed the IOA form following the collection of data (see Appendix E). The IOA form was a summary form that allowed for easy comparison of the data collected on the same session by the 
researcher and the second observer. The formula used to calculate IOA was printed at the bottom of the form, allowing IOA for each observation to be calculated.

\section{Data Collection Forms}

The data collection forms were used to record participant performance (see Appendix F) by the researcher and the independent observer. Each form contained a reading passage of approximately 100 words that was typed in 14-point font and aligned left. The forms were printed on $8.5 " \mathrm{x} 11$ " plain white copy paper with 1" margins on each side. Below the passages were 2 spaces in which the raters could write the total correct words read per minute (CWPM) and errors per minute (EPM). Finally, the forms had the five questions pertaining to the reading passage printed on them with a "yes" or "no" next to each question to record correct and incorrect answers.

\section{Informal Reading Inventory}

The Analytical Reading Inventory (Woods \& Moe, 2006) was the informal reading inventory used in the study. This informal reading assessment examined oral reading and reading comprehension skills of the participants prior to the start of the study. The responses were coded for word recognition and categorized as independent (99-100\% accuracy), instructional (91-98\% accuracy), or frustration (90\% or below). The comprehension scores were coded as independent (90-100\% accuracy), instructional (75$89 \%$ accuracy), or frustration ( $74 \%$ or below). This informal reading inventory was used to determine the overall instructional reading level of each participant. The passages used throughout the study were at the instructional reading level of each participant. 


\section{Fry Readability Graph}

The Fry Readability Graph (Fry et al., 1985) was used to verify the grade reading level of the passages selected from the basal reading series. The graph uses the average number of sentences (y-axis) and syllables (x-axis) per hundred words. These averages were plotted and the intersection of the average number of sentences and the average number of syllables determined the reading level of the passage.

\section{Reading Passages}

Approximately 60 passages from the Houghton Mifflin Invitations to Literacy Series (1998) were chosen for each student at their instructional reading level as indicated by the ARI score. These passages were randomly assigned to the baseline and intervention phases. The passage reading levels were verified both by the publisher, as well as by the readability graph developed by Fry (1985). Passages were retyped to facilitate the reading of continuous text and to eliminate picture cues (Alber-Morgan et al., 2007). The passages were approximately 100 words (with a minimum of 100 and a maximum of 105 words) and were typed using double spacing and 1" margins in 14-point Times New Roman font on 8.5" x 11" plain white copy paper. The passages were aligned on the left hand side of the page.

\section{Flashcards of Challenging Words}

Each passage contained one to five words deemed as challenging by the basal reading key. These words were printed in 14 point Times New Roman font on 3" x 5" plain white flashcards for use in vocabulary instruction. The definitions of these words, taken directly from the basal series glossary, were printed on the back of these flashcards. Additionally, a sentence using each challenging word was printed on the back of the card. 


\section{Literal Comprehension Questions}

Each passage had five corresponding literal comprehension questions that required the student to recall items that were explicitly mentioned in the text. These questions were adapted from the workbooks and teacher's editions of the basal reading series. A district reading curriculum and instruction specialist checked each question and determined that the answer was found in the text. The questions were typed on a paper that only the researcher could see during the sessions.

\section{Generalization Passages}

The researcher created generalization passages prior to the start of the study. These passages had approximately $80 \%$ of the same words as the reading passages. The readability of these passages was verified by the Fry Readability Graph (Fry et al., 1985) to ensure that they were at the appropriate instructional reading level. The passages were approximately 100 words (with a minimum of 100 and a maximum of 105 words) and were typed using double spacing and 1" margins in 14 point Times New Roman font on 8.5 " $\mathrm{x} 11$ " plain white copy paper. The passages were aligned on the left hand side of the page. Five literal comprehension questions were written for these generalization passages. A reading curriculum and instruction specialist from the school district verified that the questions are of the same difficulty level as the questions used in the intervention passages.

\section{Digital Recording Device}

An Olympus digital recorder with a built in timer was used to make a permanent product of each session. Both the researcher and the independent raters analyzed the recorded sessions. The recorder had a computer compatible cable that allowed audio files 
to be archived in the researcher's computer and sent to the independent raters as e-mail attachments. This allowed the researcher to score the reading assessments as well as to have a record that the independent raters used to ensure IOA and treatment fidelity.

Dependent Variables

Each session in this study was recorded and transferred to a digital file. This allowed for precise measurement of the dependent variables. The dependent variables were as follows: (a) reading fluency as measured by the number of correct words read aloud per minute, (b) the number and types of errors read aloud per minute of reading, (c) number of literal comprehension questions answered correctly aloud, (d) generalization of gains made to unfamiliar passages, and (e) maintenance of performance after 2-, 4-, and 6-weeks.

Reading Fluency as Measured by Correct Words per Minute (CWPM)

Reading fluency was defined as the number of words read correctly aloud per minute of reading. Participant self corrections were scored as correct. A word was counted as correct if the participant independently pronounced it correctly aloud without prompting within 3 seconds (Nelson et al., 2004).

Number and Types of Errors per Minute (EPM)

This is the total number of errors read aloud during a 1-minute recording (Tam et al., 2006). Data on the following error types were collected:

Omission. Defined as a printed word in the passage that was not read aloud by the participant.

Addition. Defined as a word that was read aloud by the participant but was not printed in the passage. 
Mispronunciation. Defined as a printed word that was read aloud incorrectly. For example if the text said "he knows many Latin words" but the participant read aloud, "He knows many Lateen words" (Dictionary.com, 2008).

Substitution. Defined as a word that was read aloud different than the printed word. For example, the text read, "He saw the park." The participant read, "He saw the pumpkin."

Hesitation. Defined as a delay in reading aloud of more than 3 seconds from the end of one word read aloud to the beginning of the next word read aloud. The researcher timed the seconds by counting silently. Once the 3 seconds had passed, the researcher read the word correctly so that the participant could read on.

\section{Literal Comprehension Question Assessment}

After completing the passage readings, five literal comprehension questions were asked by the researcher. The participant had 5 seconds to answer the question. Participant responses were compared with the answer key provided by the basal reader for that reading passage. Correct responses were those that match the answer key and that were stated aloud within 5 seconds after the end of the question. Responses that did not match the answer key or those begun more than 5 seconds after the end of the question were scored as incorrect. The total number of correct responses were recorded and graphed.

\section{Generalization}

Immediately following approximately $25 \%$ of the sessions throughout the study, generalization probes were taken to test generalization of skills to untaught passages that were similar to those being used in experimental sessions. Generalization passages were approximately 100 words (with a minimum of 100 words and a maximum of 105 words) 
in length and were of the same level as those being used in experimental sessions according to the Fry Readability Graph (Fry et al., 1985). Additionally, approximately $80 \%$ of the words in the generalization passage came directly from the passage that was used in the experimental session. Generalization probes included a single reading of the passage without error correction feedback from the researcher. Generalization probes also included data collection in the form of a fluency assessment and a literal comprehension question assessment as defined previously.

\section{Maintenance}

Maintenance was defined as performance on passage assessments given 2-, 4-, and 6-weeks after the study concluded. Maintenance probes included a single reading of a passage without error correction feedback from the researcher. Maintenance probes also included data collection in the form of a fluency assessment and a literal comprehension question assessment as defined previously. Passages used during the experimental session were used during maintenance allowing for a direct comparison of the experimental and maintenance sessions. Maintenance passages were randomly selected from the passages used during the first two-thirds of the experimental sessions. This way, the passage selected was not the most recently taught.

\section{Interobserver Agreement}

Two independent raters were trained to listen to the recordings and count the number of correct words read aloud per minute (CWPM), the number of errors read aloud per minute (EPM), and the types of errors read aloud per minute. These raters also listened to the responses to the comprehension questions and scored them as correct or 
incorrect in accordance with the basal key. The raters were undergraduate students enrolled in an elementary education program at Florida International University.

Training of the independent raters was provided by the researcher and lasted approximately 1 hour. The training session consisted of an oral discussion and a written handout of the procedures for scoring the audio recordings (see Appendix E). Following this the raters and the researcher independently scored two audio samples that had been previously scored by an expert in the field (a district reading curriculum and instruction specialist). The researcher and the raters then compared their scores with the expert and with each other. Both reviewed the criterion and practiced scoring samples until at least $90 \%$ agreement on the sample was reached with the expert. At that point, the independent raters were considered adequately trained.

Throughout the study, independent raters scored approximately $25 \%$ of randomly selected audio recordings. Interobserver agreement (IOA) data were taken in the baseline, intervention, generalization and maintenance conditions. Agreements and disagreements were counted using a word-by-word examination of the data sheets. IOA was calculated by dividing the number of agreements by the number of agreements plus disagreements and then multiplying by 100. A minimum mean IOA of $90 \%$ was calculated for each individual participant. IOA did not fall below 90\%, making retraining of the researcher and observers unnecessary.

\section{Treatment Fidelity}

The researcher gathered a treatment fidelity measure daily during each session. Treatment fidelity refers to the extent to which the treatment, or intervention, is carried out according to what is written in the methods section of the study in question (Cooper 
et al., 2007). Monitoring treatment fidelity allowed for a record of the researcher's consistency. It served as a routine review of the procedures as well as a way to determine problems in implementation before they became habitual. This measure was taken by using a checklist to record the daily occurrence and nonoccurrence of the planned procedures.

The same individuals trained for IOA were trained to collect treatment fidelity data to help ensure accuracy of the procedural implementation as designed. Training was provided in a 1-hour session. During the training session, the rater was given a treatment fidelity checklist (see Appendix D) and asked to listen to an audio recording of the researcher executing the steps with a student. The rater's checklist was then compared to the researcher's checklist for that session in order to insure that the researcher was accurately self-assessing her implementation of the written procedure. This procedure was repeated until the observer and the researcher were in agreement for every step on the checklist. At this point, the observers were considered properly trained.

After the training session, the observer listened to approximately $25 \%$ of randomly selected sessions. The observer independently scored the occurrence and nonoccurrence of the planned procedures. The total percentage of occurrence and nonoccurrence of the planned procedures was recorded. Data were collected during the baseline, generalization, and intervention conditions. The treatment fidelity forms completed by the researcher and the independent raters was analyzed for the percentage of adherence to the planned procedure and reported in the discussion section of this study. 


\section{Experimental Design}

A multiple probe baseline design across subjects was used to examine the effects of repeated readings on the number of words read aloud correctly per minute, the numbers and types of errors read aloud per minute, and the generalization and maintenance of performance by ELLs with SLD. In this design, once steady state responding is reached under baseline probes, the intervention is applied to one participant while the other participants remain in baseline. When steady state is reached in intervention for the first participant, the same intervention is applied to next participant and so on. In contrast to a multiple baseline design, in which baseline data are collected throughout the baseline condition, in a multiple probe baseline design, probes (or periodic measures) are taken until a few days prior to implementation of the intervention at which time continuous measures are taken. The probes provide the basis for prediction and determining whether behavior change has occurred prior to intervention.

The multiple probe baseline design across subjects was used in order to limit the potential practice effects. Practice effects occur when there is improvement due to the increased opportunities to emit the target behavior (Cooper et al., 2007), creating an unwanted confound. In this study, at least four data points were collected during baseline before the implementation of the intervention. These points demonstrated that the behavior was unlikely to change without the introduction of the intervention.

In a multiple probe baseline design, data analysis is accomplished through the visual inspection of graphed data in which performance during the intervention is compared to performance during baseline. Predictions based on one participant's behavior are verified by the performance of the other participants, and replication of 
effect is dependent on the performance of other participants. Verification is evident if the data path changes in a predictable manner through a condition change, as from baseline to intervention for each participant. Replication of this prediction and verification may occur when the data paths of the other participants follow patterns similar to the first participant.

A functional relation is identified when baseline behaviors are stable and change only when the intervention is applied. Convincing relations are demonstrated by clear differences in responding with a minimal overlap of data paths across conditions. Experimental control is demonstrated by replicating the effects with another participant (Cooper et al., 2007).

\section{General Procedures}

The following describes the procedures used in this study. The pre-study procedures section describes the steps taken prior to the start of the study including the selection of the participants, obtaining parent consent, obtaining participant assent, an informal assessment of reading and comprehension skills, and second observer selection and training.

This is followed by a description of how the reading materials were prepared prior to the start of the sessions. The general study procedures section includes the procedures that were used both in the baseline and intervention conditions. The generalization and maintenance sections describe how measures were taken to examine the effects of this study. 


\section{Pre-Study Procedures}

The pre-study procedures were the steps implemented prior to the beginning of data collection on the dependent variables. These procedures were as follows:

Selection of participants. The researcher obtained permission from the district and the principal to visit the school and schedule a meeting for the general education, special education, and ESOL teachers that work with ELLs at levels III, IV, and V. At this meeting, the researcher described the study and the criterion for nominating students as participants. The teachers were asked to nominate ELLs identified as having SLD who were reading at least one year below grade level in English. The teachers were told that their nominations must be based on their experiences with the participants and the participants' performance in reading tasks in their classes. The researcher scheduled a time later that week with the teachers and collected 10 names and respective grade levels of prospective participants.

Parent consent. A consent form in English and Spanish was sent home with the 10 nominated, potential participants. The parents were given 3 days to sign and return the form or contact the researcher with questions. Parents who declined were removed from the list of potential participants. Follow up calls were made after 3 days to the parents who had not responded and to the parents who agreed to allow their child to participate. Parents who had not responded were called in order to discuss their child's potential participation and answer any questions they may have. Parents who had returned the form giving permission to participate were called in order to discuss the study and to answer any questions they may have. The researcher, who is bilingual, called both English and Spanish speaking parents. 
Participant assent. A form similar to the parent consent was read to the potential student participants. This occurred during a one-on-one meeting with the researcher. This form was written in language that was developmentally appropriate for the potential participants and it discussed the study and the roles of the researcher and participants. The researcher answered any participant questions. The potential participants were asked to sign the form assenting to participate in the study and were given a copy of the signed form.

Informal assessment of reading and comprehension skills. One week prior to starting the study, the Analytical Reading Inventory (Woods \& Moe, 2006) was individually administered to each participant. Participants read aloud grade level passages and answered questions while the researcher recorded errors. The oral reading responses were then coded and categorized as independent (99-100\% accuracy), instructional (9198\% accuracy), or frustration (90\% or below). The comprehension scores were coded as independent (90-100\% accuracy), instructional (75-89\% accuracy), or frustration (74\% or below). The overall instructional reading scores, which took into consideration the oral reading and comprehension scores, were used to determine the level of the reading passages that were used with that participant throughout the study.

Second observer selection and training. Three independent observers were recruited from an undergraduate elementary education course at Florida International University. To do this, the researcher requested permission to attend the undergraduate class and give a brief presentation summarizing the duties of the second observer. A page with lines for names, e-mail addresses, and telephone numbers of interested candidates was circulated. The researcher collected the circulated list. The researcher then called and 
interviewed the interested individuals. This telephone interview helped the researcher determine if the individual had enough time and dedication to the study. From this effort, two individuals were selected for training and participation as second observers and two individuals were selected as alternates.

Reading material preparation. The reading passages were chosen from the Houghton Mifflin Invitations to Literacy Series (1998) to which there had been no prior participant exposure. Additionally, they did not have any exposure to this basal during the course of the study. The researcher chose approximately 60 passages for each student at his or her independent reading level as indicated by the ARI score. The reading levels of the materials were verified both by the publisher's provided key, as well as by the readability graph developed by Fry (1985).

The researcher retyped the basal reader passages in 14-point Times New Roman font using double spacing. The passages were approximately 100 words (with a minimum of 100 words and a maximum of 105 words) and were printed on 8.5 " x 11 " plain white copy paper with 1" margins on all four sides and a left alignment. The passages were randomly assigned to baseline or intervention conditions.

Then, the researcher developed and typed the generalization passages. The Fry Readability Graph (Fry et al., 1985) was used to ensure that the readability level was the same as the passages used in the baseline and intervention phases, as well as to ensure that at lease $80 \%$ of the words in the generalization passages were also found in the baseline and intervention phase passages. The generalization passages each had five corresponding literal comprehension questions that were written by the researcher and verified by the district reading curriculum and instruction specialist to ensure that they 
were of the same level of difficulty as those used during the baseline and experimental sessions. Maintenance passages were taken randomly from passages used during the intervention phase.

The room where the sessions took place was prepared prior to the participant's arrival. A blank file folder with the passage for the day was waiting at the desk where the participant was to sit. The researcher also had a blank file with the treatment fidelity form, the passage of the day, the flashcards of the challenging words, and the corresponding literal comprehension questions.

\section{Multiple Probe Baseline Condition}

In the multiple probe baseline condition, the researcher led each participant in an introduction to the passage of the day in the form of vocabulary instruction. The researcher used flashcards to determine if the participant knew the meaning of the challenging words in the upcoming reading. These words were selected by the basal reading series, which deems certain words in the passages as challenging. The researcher taught the words not known to the student. The teaching consisted of the researcher asking the participant to read the word aloud. If the participant could not do this, the researcher stated the word and asked the participant to repeat it aloud and use the word in a sentence. If the participant used the word correctly, the researcher praised the participant and moved on to the next word. If the participant could not use the word in a sentence, the researcher read the definition from the back of the card and then asked the participant to use the word in a sentence aloud. If the participant still could not do this, the researcher used the word in a sentence that demonstrated its meaning. This lasted approximately 1 to 3 minutes depending on the number of words the student knew. 
Next, the participant was asked to read the passage. During this reading, a whole word error correction strategy was employed (Barbetta et al., 1993). With whole word error correction, when a participant made a reading error, the researcher immediately read the word correctly. Following that, the participant repeated the word aloud. After the repetition of the word, the participant repeated the entire sentence containing that word aloud. Once the passage was read aloud in its entirety, the words that were initially stated incorrectly were repeated aloud by the participant again in isolation. The participant then was asked to read the passage aloud from the beginning for a 1-minute fluency assessment, which assessed CWPM, EPM, and type of EPM.

After the fluency reading assessment, a literal comprehension question assessment occurred. This consisted of five literal comprehension questions asked orally by the researcher. The researcher asked the five questions aloud, one question at a time. The participant was given 5 seconds to respond to each question aloud. If the participant responded with the correct answer, the researcher made a short positive statement (such as "yes" or "correct"). If the participant responded incorrectly, the researcher provided error correction (e.g., "No, the cow jumped over the moon, not the fence."). Following the final question and student response the researcher issued a statement of gratitude (e.g., "thank you for working hard today"), indicating the end of the session.

\section{Repeated Readings Intervention Condition}

The repeated readings intervention condition was identical to the multiple probe baseline condition for the initial reading. In addition, each session contained repeated readings (two additional readings) of the passage for a total of three readings. Following the initial reading of the passage, the researcher asked the participant to read the passage 
aloud two more times from the beginning. The two additional passage readings did not include the error correction procedures explained in baseline. Instead, when an error was emitted, the researcher said the correct word, but did not prompt the student to repeat the word or the sentence containing the word (Alber-Morgan et al., 2007; Nelson et al., 2004; Tam et al., 2006). The fluency assessment and literal comprehension question assessment followed using the same procedure as described in baseline.

\section{Generalization}

Generalization probes were administered immediately following approximately $25 \%$ of the baseline and intervention sessions. A new passage, at the same readability level and with $80 \%$ of the same words as that day's session passage were prepared. The generalization session consisted of the student reading the passage aloud once. This reading did not include the error correction procedures explained in baseline. Following the single reading of the passage, the fluency and literal comprehension question assessments occurred as they did in the other phases.

\section{Maintenance}

Maintenance data were collected 2-, 4-, and 6-weeks after the last repeated readings intervention session. Passages used during the first two-thirds of the repeated readings intervention sessions were randomly selected for use during maintenance. Similar to the generalization probes, the participant read the passage once aloud during maintenance sessions. This reading did not include the error correction procedures explained in baseline. Following the single reading of the passage, the fluency and literal comprehension question assessments occurred as they did in the other phases. 


\section{Summary}

This study examined the effects of repeated readings on reading fluency, reading errors, and responses to literal comprehension questions of third-, fourth-, and fifth-grade ELLs with SLD who are struggling readers (defined as reading at a level at least one year below grade level in English). The research questions focused on whether or not the repeated readings intervention had an effect on the number of CWPM, the number and types of EPM, and the maintenance and generalization of skills attained during intervention.

The participants, nominated by their teachers, were four ELLs with SLD who are struggling readers enrolled at, a large, urban, public elementary school. The dependent variables were reading fluency as measured by the number of correct words read aloud per minute and the number and types of errors read aloud per minute. Additionally, examined was the number of correct answers to literal comprehension questions. The study also collected data on the generalization and maintenance of the repeated reading intervention on the dependent variables. IOA and treatment fidelity data were collected.

A single subject, multiple probe baseline design across subjects was used in this investigation. This design was appropriate because it allowed for baseline collection of data without too many baseline sessions in an effort to limit practice effects. In this multiple probe baseline design, steady state responding was reached in continuous baseline before applying the intervention to the first participant. The remaining participants were administered intermittent baseline probes until steady state responding was reached with the first participant in intervention. Prior to intervention on subsequent participants, a minimum of four days of continuous baseline data were collected. The 
intervention was then applied to the next participant and so on. Data analysis was done through visual examination of data graphs and verification occurred when data paths changed predictably when each participant received the intervention. A functional relation occurred when baseline behaviors changed only when the intervention was applied.

Pre-study procedures began with obtaining written permission from the school district and the school principal and meeting the teachers at the elementary school. The teachers recommended 10 potential participants. From these recommendations the researcher selected the students who met the criteria of being ELLs at Levels III, IV, or $\mathrm{V}$, identified as having SLD and struggling with reading. These students received forms, followed by telephone calls to obtain parental permission. After parental permission was obtained and verified, the potential participants and the researcher read and discussed the participant assent form. Following this, an informal reading assessment was conducted on each participant. The second observers were selected and trained. The reading materials were also be prepared, organized, and randomly assigned to either baseline or intervention conditions prior to the start of the study.

The researcher conducted the study in a quiet room in the school's media center. Sessions were conducted one-on-one during 10 to 20 minute scheduled sessions. During the multiple probe baseline condition the participants received vocabulary instruction and then they read aloud a passage while receiving corrective feedback. Immediately after this, fluency and literal comprehension question assessments were given. The repeated readings intervention condition included the same vocabulary instruction, and initial passage reading with error correction feedback. Following this, the participants 
repeatedly read aloud the same passage two additional times without error correction feedback. Immediately after this, a fluency assessment and literal comprehension question assessment were given.

Generalization probes were taken immediately following approximately $25 \%$ of the sessions using passages during intervention. Generalization probes consisted of the participant reading aloud the passage once without error feedback from the researcher. Next, the participant read aloud for a 1-minute fluency assessment, and answered a five question literal comprehension assessment. Maintenance probes were done on previously read passages and followed the same procedures as the generalization probes. Maintenance data were collected at 2-, 4-, and 6-weeks after the end of the study. All data were graphed to facilitate visual examination. 


\section{CHAPTER IV}

\section{RESULTS}

This chapter presents the findings of a study that used a multiple probe baseline design across subjects to examine the effects of repeated readings on reading fluency, reading errors, and reading comprehension of English language learners (ELLs) with specific learning disabilities (SLD). The study sought to answer the research questions by measuring the number of correct words read aloud per minute (wpm), the number of errors read aloud per minute (epm), the types of errors read aloud per minute, and the number of literal comprehension questions answered correctly. Additionally, this study examined if the participants generalized any observed gains to new passages with similar words and maintained any observed gains over a period of 2-, 4-, and 6-weeks following the final intervention session.

Treatment fidelity and interobserver agreement (IOA) data are presented first. These data are followed by the results on the each participant's performance and group means on the dependent variables. This chapter concludes with a summary of the results.

\section{Treatment Fidelity}

The researcher and two trained independent observers collected treatment fidelity data to help ensure that procedures were followed as designed. The researcher collected procedural data in every session (100\%) across all participants and conditions. The researcher data indicated that procedures were followed an average of $99.87 \%$ of the time (range 92.59-100) throughout all of the sessions. The independent observers collected treatment fidelity data on 51 of 204 or $25 \%$ of the sessions across all participants and all 
conditions. The independent observers data indicated that procedures were followed an average of $99.78 \%$ of the time (range $92.59-100$ ).

\section{Interobserver Agreement}

Two trained observers collected interobserver agreement data for $25 \%$ of all sessions across all dependent variables. The mean interobserver agreement for fluency was $98.39 \%$ (range 91.42-100). The mean interobserver agreement for number of errors per minute was $94.87 \%$ (range 66.67-100). The mean interobserver agreement for types of errors per minute was $90.31 \%$ (range 50-100). The mean interobserver agreement for literal comprehension questions answered correctly was 98.97\% (range 80-100).

\section{Reading Fluency}

This study was conducted, in part, to examine the effects of repeated readings on the oral reading fluency of ELLs with SLD. Oral reading fluency was measured by counting the number of words read aloud correctly per minute during a fluency assessment at the end of each session. Data on reading fluency are presented in the form of individual daily words per minute performance and individual words per minute means and ranges.

Generalization scores for fluency are presented in the form of words per minute means and ranges. The results from the generalization probes are then compared to the performance means and ranges of the preceding regular sessions. These regular sessions are referred to as the comparison score. Maintenance probes were administered 2-, 4-, and 6-weeks following the last intervention session across all conditions and participants. The probes consisted of a single reading of a passage followed by a fluency and 
comprehension assessment. The results from the maintenance probes are then compared to the performance in the last intervention session.

\section{Tano}

Figure 1 (page 69) displays Tano's performance in reading fluency during baseline, repeated readings, generalization probes, and maintenance probes. During baseline, Tano's mean reading fluency was $68.62 \mathrm{wpm}$ (range 57-76). When repeated readings was introduced, Tano's mean reading fluency increased to $85.83 \mathrm{wpm}$ (range 54-97). Tano's reading fluency was highest during the repeated readings intervention, with a mean increase of 17.21 wpm over baseline (see Table 2, page 70).

Generalization probes were completed following $26.5 \%$ of Tano's sessions (see Table 3, page 71). During baseline, Tano's mean reading fluency comparison score was 62.5 wpm (range 57-68). His mean reading fluency score for baseline generalization was 72 wpm (range 70-74). In baseline, his mean generalization score was 9.5 wpm higher than his comparison probe score.

During intervention, Tano's mean reading fluency comparison score was 87 wpm (range 78-96). His mean reading fluency scores for intervention generalization was 81.4 wpm (range 73-88). In intervention, his mean generalization score was 5.6 wpm lower than his comparison probe score.

During generalization maintenance, Tano's reading fluency comparison score was $76 \mathrm{wpm}$. His reading fluency score for maintenance generalization was $84 \mathrm{wpm}$. In maintenance, his generalization score was 8 wpm higher than his comparison probe score. 
Reading Fluency
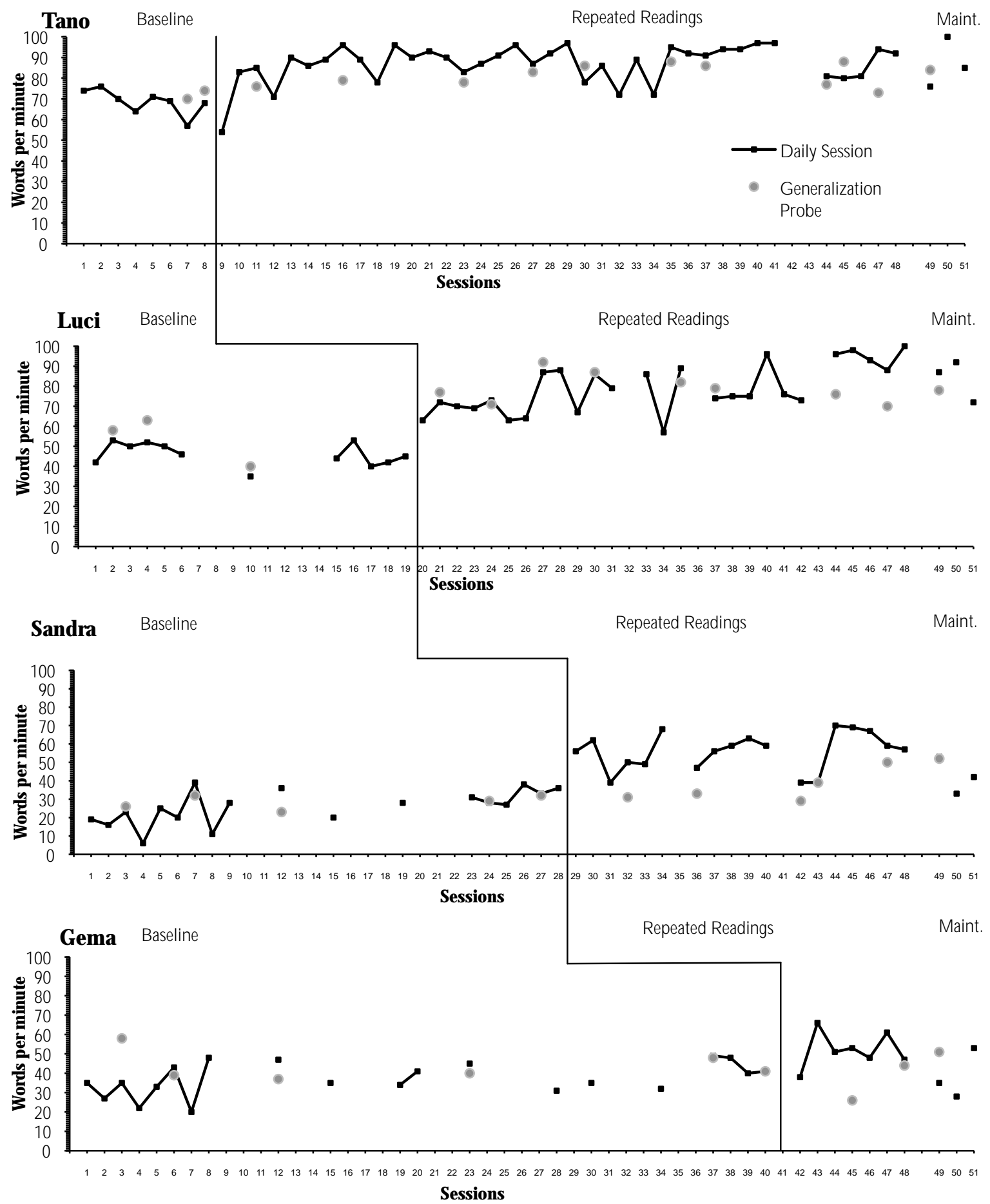

Figure 1. Reading fluency as measured by the number of words read correctly per minute during a 1-minute fluency assessment at the end of each session. Maintenance (Maint.) occurred 2-, 4-, and 6-weeks after the last intervention session. 
Table 2

Individual Means on Reading Fluency

\begin{tabular}{ccc}
\hline Participant & Baseline & Repeated Readings \\
\hline \multirow{2}{*}{ Tano } & 68.62 & 85.83 \\
& $(57-76)$ & $(54-97)$ \\
Luci & 46.09 & 76.57 \\
& $(35-53)$ & $(57-100)$ \\
Sandra & 25.77 & 53.85 \\
& $(6-39)$ & $(39-70)$ \\
Gema & 37.05 & 52.0 \\
& $(20-49)$ & $(38-66)$ \\
Group & 39.46 & 75.7 \\
\hline
\end{tabular}

Note. Top numbers indicate individual mean of words read per minute. The bottom rows of numbers represent the range of scores.

In comparing baseline, intervention, and maintenance reading fluency generalization scores, Tano read an average of $9.4 \mathrm{wpm}$ more during intervention generalization probes than baseline generalization probes. Tano's reading fluency was highest on his maintenance generalization probe with $84 \mathrm{wpm}$.

Table 4 (page 72) displays Tano’s maintenance performance. Tano’s fluency score on his last day of intervention was $92 \mathrm{wpm}$. He had maintenance scores of 76, 100, and 85 wpm respectively. Overall, Tano maintained between $82.61 \%$ (week 2 probe) and $108.7 \%$ (week 4 probe) of his reading fluency. Tano performed highest on his week 4 maintenance probe. 
Table 3

Mean Reading Fluency in Comparison Sessions and Generalization Probes

\begin{tabular}{|c|c|c|c|c|}
\hline \multirow{2}{*}{\multicolumn{2}{|c|}{ Participant }} & \multicolumn{3}{|c|}{ Words Per Minute } \\
\hline & & Baseline & Repeated Readings & Maintenance \\
\hline \multirow[t]{2}{*}{ Tano } & $\begin{array}{l}\text { Comparison } \\
\text { Scores }\end{array}$ & $\begin{array}{c}62.5 \\
(57-68)\end{array}$ & $\begin{array}{c}87 \\
(78-96)\end{array}$ & 76 \\
\hline & $\begin{array}{l}\text { Generalization } \\
\text { Probes }\end{array}$ & $\begin{array}{c}72 \\
(70-74)\end{array}$ & $\begin{array}{c}81.4 \\
(73-88)\end{array}$ & 84 \\
\hline \multirow[t]{2}{*}{ Luci } & $\begin{array}{l}\text { Comparison } \\
\text { Scores }\end{array}$ & $\begin{array}{l}46.67 \\
(35-53)\end{array}$ & $\begin{array}{c}83.13 \\
(72-96)\end{array}$ & 87 \\
\hline & $\begin{array}{c}\text { Generalization } \\
\text { Probes }\end{array}$ & $\begin{array}{c}53.67 \\
(40-63)\end{array}$ & $\begin{array}{c}79.25 \\
(70-92)\end{array}$ & 78 \\
\hline \multirow[t]{2}{*}{ Sandra } & $\begin{array}{l}\text { Comparison } \\
\text { Scores }\end{array}$ & $\begin{array}{c}31.8 \\
(23-39)\end{array}$ & $\begin{array}{c}46.8 \\
(39-59)\end{array}$ & 53 \\
\hline & $\begin{array}{l}\text { Generalization } \\
\text { Probes }\end{array}$ & $\begin{array}{c}28.4 \\
(26-32)\end{array}$ & $\begin{array}{c}36.4 \\
(29-50)\end{array}$ & 52 \\
\hline \multirow[t]{2}{*}{ Gema } & $\begin{array}{l}\text { Comparison } \\
\text { Scores }\end{array}$ & $\begin{array}{c}43.33 \\
(35-49)\end{array}$ & $\begin{array}{c}50 \\
(47-53)\end{array}$ & 35 \\
\hline & $\begin{array}{l}\text { Generalization } \\
\text { Probes }\end{array}$ & $\begin{array}{c}43.83 \\
(39-58)\end{array}$ & $\begin{array}{c}35 \\
(26-44)\end{array}$ & 51 \\
\hline \multirow[t]{2}{*}{$\begin{array}{l}\text { Group } \\
\text { Means }\end{array}$} & $\begin{array}{l}\text { Comparison } \\
\text { Scores }\end{array}$ & $\begin{array}{c}39.76 \\
(23-68)\end{array}$ & $\begin{array}{c}75.7 \\
(39-96)\end{array}$ & $\begin{array}{c}62.75 \\
(35-87)\end{array}$ \\
\hline & $\begin{array}{l}\text { Generalization } \\
\text { Probes }\end{array}$ & $\begin{array}{l}44.38 \\
(26-74)\end{array}$ & $\begin{array}{c}68 \\
(26-92)\end{array}$ & $\begin{array}{c}66.25 \\
(51-84)\end{array}$ \\
\hline
\end{tabular}

Note. Comparison scores are taken from that day's regular session. Generalization probes immediately followed the regular session. Top numbers indicate mean number of words read per minute. The bottom rows of numbers represent the ranges of scores.

Luci

Figure 1 (page 69) displays Luci’s performance in reading fluency during baseline, repeated readings, generalization probes, and maintenance probes. During baseline, Luci's mean reading fluency was 46.09 wpm (range 35-53). When repeated readings was introduced, Luci's mean reading fluency increased to $76.57 \mathrm{wpm}$ (range of 
57-100). Luci's reading fluency was highest during the repeated readings intervention, with a mean increase of 30.48 wpm over baseline (see Table 2, page 70).

Table 4

Maintenance on Reading Fluency

\begin{tabular}{lcccc}
\hline Participant & $\begin{array}{c}\text { Last Intervention } \\
\text { Score }\end{array}$ & $\begin{array}{c}\text { Week } 2 \\
\text { Probe }\end{array}$ & $\begin{array}{c}\text { Week } 4 \\
\text { Probe }\end{array}$ & $\begin{array}{c}\text { Week 6 } \\
\text { Probe }\end{array}$ \\
\hline Tano & 92 & 76 & 100 & 85 \\
& & $82.61 \%$ & $108.7 \%$ & $92.39 \%$ \\
Luci & 100 & 87 & 92 & 72 \\
& & $87 \%$ & $92 \%$ & $72 \%$ \\
Sandra & 57 & 53 & 33 & 42 \\
& 47 & $92.98 \%$ & $57.89 \%$ & $73.68 \%$ \\
Gema & & 35 & 28 & 53 \\
& & $74.47 \%$ & $59.57 \%$ & $112.77 \%$ \\
Group Means & 74 & 62.75 & 63.25 & 63 \\
& & $84.8 \%$ & $85.47 \%$ & $85.14 \%$ \\
\hline
\end{tabular}

Note. Top numbers indicate individual number of words read per minute on the last day of treatment and on 2-, 4- and 6-week maintenance probes. The bottom row percentages indicate the percentage of reading fluency maintained on 2-, 4- and 6-week maintenance probes.

Generalization probes were completed following $29.26 \%$ of Luci's sessions (see Table 3, page 71). During baseline, Luci's mean reading fluency comparison score was 46.67 wpm (range 35-53). Her mean reading fluency score for baseline generalization was 53.67 wpm (range 40-63). In baseline, her mean generalization score was 7 wpm higher than her comparison probe score.

During intervention, Luci's mean reading fluency comparison score was 83.13 wpm (range 72-96). Her mean reading fluency scores for intervention generalization was 
79.25 wpm (range 70-92). In intervention, her mean generalization score was 3.88 wpm lower than her comparison probe score.

During maintenance, Luci's reading fluency comparison score 87 wpm. Her reading fluency score for maintenance generalization was 78 wpm. In maintenance, her generalization score was $9 \mathrm{wpm}$ lower than her comparison probe score.

In comparing baseline, intervention and maintenance reading fluency generalization scores, Luci read an average of $25.58 \mathrm{wpm}$ more wpm during intervention generalization probes than baseline generalization probes. Luci's mean generalization reading fluency was highest during intervention generalization probes with $79.25 \mathrm{wpm}$.

Table 4 (page 72) displays Luci’s maintenance performance. Luci’s fluency score on her last day of intervention was $100 \mathrm{wpm}$. She had maintenance scores of 87, 92, and 72 wpm respectively. Overall, Luci maintained between $72 \%$ (week 6 probe) and $92 \%$ (week 4 probe) of her reading fluency. Luci performed highest on her week 4 maintenance probe.

Sandra

Figure 1 (page 69) displays Sandra's performance in reading fluency during baseline, repeated readings, generalization probes and maintenance probes. During baseline, Sandra's mean reading fluency was 25.77 wpm (range 6-39). When repeated readings was introduced, Sandra's mean reading fluency increased to 53.85 wpm (range 39-70). Sandra's reading fluency was highest during the repeated readings intervention, with a mean increase of 28.08 wpm over baseline (see Table 2, page 70).

Generalization probes were completed following $28.21 \%$ of Sandra's sessions (see Table 2, page 70). During baseline, Sandra's mean reading fluency comparison score 
was 31.8 wpm (range 23-39). Her mean reading fluency score for baseline generalization was 28.4 wpm (range 26-32). In baseline, her mean generalization score was 3.4 wpm lower than her comparison probe score.

During intervention, Sandra's mean reading fluency comparison score was 46.8 wpm (range 39-59). Her mean reading fluency scores for intervention generalization was 36.4 wpm (range 29-50). In intervention, her mean generalization score was 10.4 wpm lower than her comparison probe score.

During maintenance, Sandra's reading fluency comparison score 53 wpm. Her reading fluency score for maintenance generalization was $52 \mathrm{wpm}$. In maintenance, her generalization score was $1 \mathrm{wpm}$ lower than her comparison probe score.

In comparing baseline, intervention, and maintenance reading fluency generalization scores, Sandra read an average of 8 more wpm during intervention generalization probes than baseline generalization probes. Sandra's reading fluency was highest on her maintenance generalization probe with $52 \mathrm{wpm}$.

Table 4 (page 72) displays Sandra's maintenance performance. Sandra's fluency score on her last day of intervention was 57 . She had maintenance scores of 53, 33, and 42wpm respectively. Overall, Sandra maintained between $57.89 \%$ (week 4 probe) and $92.98 \%$ (week 2 probe) of her reading fluency. Sandra performed highest on her week 2 maintenance probe.

\section{Gema}

Figure 1 (page 69) displays Gema's performance in reading fluency during baseline, repeated readings, generalization probes, and maintenance probes. During baseline, Gema's mean reading fluency was 37.05 wpm (range 20-49). When repeated 
readings was introduced, Gema's mean reading fluency increased to 52.0 wpm (range 3866). Gema's reading fluency was highest during the repeated readings intervention, with a mean increase of $14.95 \mathrm{wpm}$ over baseline (see Table 2, page 70).

Generalization probes were completed following $28.57 \%$ of Gema's sessions (see Table 3, page 71). During baseline, Gema's mean reading fluency comparison score was 43.33 wpm (range 35-49). Her mean reading fluency score for baseline generalization was $43.83 \mathrm{wpm}$ (range 39-58). In baseline, her mean generalization score was $0.5 \mathrm{wpm}$ higher than her comparison probe score.

During intervention, Gema's mean reading fluency comparison score was 50 wpm (range 47-53). Her mean reading fluency scores for intervention generalization was 35 wpm (range 26-44). In intervention, her mean generalization score was 15 wpm lower than her comparison probe score.

During maintenance, Gema's reading fluency comparison score $35 \mathrm{wpm}$. Her reading fluency score for maintenance generalization was $51 \mathrm{wpm}$. In maintenance, her generalization score was 16 wpm higher than her comparison probe score.

In comparing baseline, intervention, and maintenance reading fluency generalization scores, Gema read an average of 8.83 fewer wpm during intervention generalization probes than baseline generalization probes. Sandra's reading fluency was highest on her maintenance generalization probe with $51 \mathrm{wpm}$.

Table 4 (page 72) displays Gema's maintenance performance. Gema's fluency score on her last day of intervention was 47 . She had maintenance scores of 35,28 , and 53 wpm respectively. Overall, Gema maintained between $59.57 \%$ (week 4 probe) and 
$112.77 \%$ (week 6 probe) of her reading fluency. Gema performed highest on her week 6 maintenance probe.

\section{Group Means for Fluency}

Table 2 displays group means for fluency during baseline and repeated readings. During baseline, the mean number of words per minute was 39.46 wpm (range 6-76). When repeated readings was introduced, the mean number of words per minute increased to 75.7 (range 38-100). Overall, the mean number of words per minute was highest during the repeated readings intervention, with $36.11 \mathrm{wpm}$ more than baseline.

Generalization probes were completed following approximately $25 \%$ of all of the sessions across all of the participants (see Table 3, page 71). During baseline, the group's mean reading fluency comparison score was 39.76 wpm (range 23-68). Their mean reading fluency score for baseline generalization was $44.38 \mathrm{wpm}$ (range 26-74). In baseline, their mean generalization score was $4.62 \mathrm{wpm}$ higher than their comparison probe score.

During intervention, the group mean reading fluency comparison score was 75.7 wpm (range 39-96). The group mean reading fluency scores for intervention generalization was 68 wpm (range 26-92). In intervention, the group mean generalization score was 7.7 wpm lower than the comparison probe score.

During maintenance, the group mean reading fluency comparison score 62.75 wpm. The group mean reading fluency score for maintenance generalization was 66.25 wpm. In maintenance, the group mean generalization score was 3.5 wpm higher than the comparison probe score. 
In comparing baseline, intervention, and maintenance reading fluency generalization scores, the group read a mean of $23.62 \mathrm{wpm}$ more during intervention generalization probes than baseline generalization probes. The group mean generalization reading fluency was highest during intervention generalization probes with $68 \mathrm{wpm}$.

Table 4 (page 72) displays the group maintenance performance. The mean group score on the last day of intervention was $74 \mathrm{wpm}$. The group had maintenance mean scores of 62.75, 63.25, and $63 \mathrm{wpm}$ respectively. Overall, the group maintained between $84.8 \%$ (week 2 probe) and $85.47 \%$ (week 4 probe) of reading fluency. The group performed highest on the week 4 maintenance probe.

\section{Number and Types of Errors Per Minute}

This study was conducted, in part, to examine the effects of repeated readings on the number and types of oral reading errors committed by ELLs with SLD. The errors were counted categorized into five types: omissions, additions, mispronunciations, substitutions, and hesitations. Data on errors per minute (epm) and type of epm are presented in the form of individual daily epm committed and individual epm means and ranges.

Generalization scores for epm are presented in the form of means and ranges. The results from the generalization probes are then compared to the epm means and ranges of the preceding regular sessions. These regular sessions are referred to as the comparison score.

Maintenance scores for epm are presented in the form of epm committed during each maintenance probe. The results from the maintenance probes are then compared to epm committed in the last intervention session. 
Tano

Number of errors per minute. Figure 2 (page 79) displays Tano's performance in the number of epm committed during baseline, repeated readings, generalization probes, and maintenance probes. During baseline, Tano's mean number of epm was 8.37 epm (range 4-16). When repeated readings was introduced, Tano's mean epm decreased to 5.21 epm (range 2-11). Tano's epm were lowest during the repeated readings intervention, with a decrease of 3.16 epm from baseline. A summary of individual mean performance on the number of epm can be found in Table 5 (page 80).

Table 6 (page 82) displays individual mean epm in comparison sessions and in generalization probes. During baseline the comparison epm score for Tano was 7 epm (range 7-7). His mean epm score for baseline generalization was 10.5 epm (range 9-12). In baseline, his mean generalization score was 3.5 epm higher than his comparison probe score.

During intervention Tano's mean epm score was 5.7 epm (range 4-8). His mean epm scores for intervention generalization was 6.4 epm (range 3-11). In intervention, his mean generalization score was a mean of 0.7 epm higher than his comparison probe score.

During maintenance, Tano's epm comparison score was 5 epm. His epm score for maintenance generalization was 6 epm. In maintenance, his generalization score was 1 epm higher than his comparison probe score. 

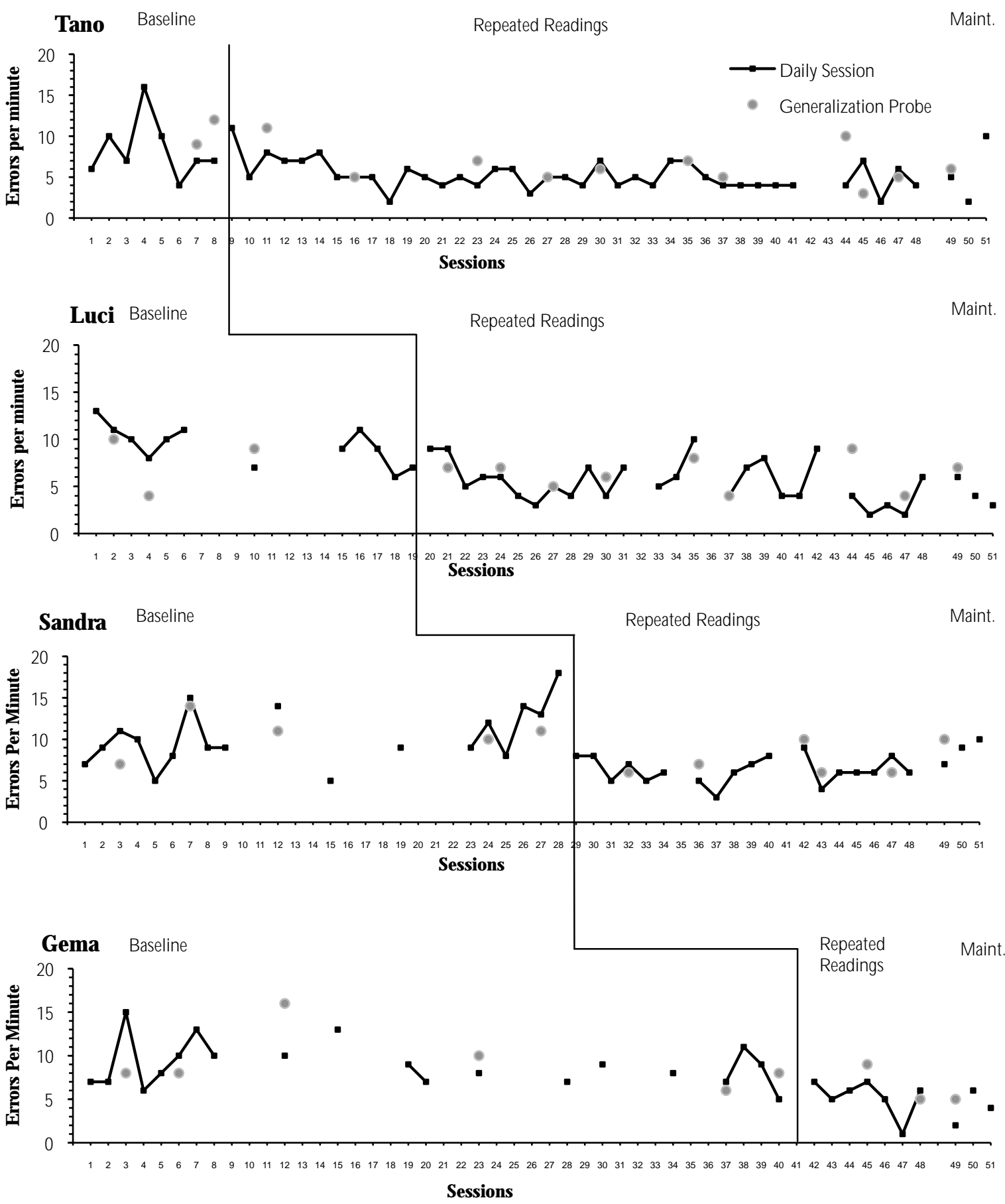

Figure 2. Errors per Minute as measured by the number of words read incorrectly per minute during a 1-minute fluency assessment at the end of each session. Maintenance (Maint.) occurred 2-, 4-, and 6-weeks after the last intervention session. 
Table 5

Individual Means on Number of Errors Per Minute

\begin{tabular}{ccc}
\hline \multirow{2}{*}{ Participant } & Baseline & Repeated Readings \\
\hline \multirow{2}{*}{ Tano } & 8.37 & 5.21 \\
& $(4-16)$ & $(2-11)$ \\
Luci & 9.54 & 5.5 \\
& $(7-13)$ & $(2-10)$ \\
Sandra & 9.77 & 6.27 \\
& $(5-18)$ & $(3-9)$ \\
Gema & 8.95 & 5.29 \\
& $(5-15)$ & $(1-7)$ \\
Group & 9.21 & 5.52 \\
& $(4-18)$ & $(1-11)$ \\
\hline
\end{tabular}

Note. Top numbers indicate individual mean of errors per minute at each phase of the intervention. The bottom rows of numbers represent the range of scores.

In comparing baseline, intervention, and maintenance epm generalization scores, Tano committed an average of 4.6 fewer epm during intervention generalization probes than baseline generalization probes. Tano's epm were lowest on his maintenance generalization probe with 6 epm.

Table 7 (page 83) displays Tano’s maintenance performance. Maintenance probes were taken 2-, 4-, and 6-weeks after the last intervention session. Tano's epm score on his last day of intervention was 4 epm. He had maintenance scores of 5, 2, and 10 epm respectively. Overall in maintenance, Tano performed between 2 epm fewer than the last session (week 4 probe) and 6 epm more than the last session (week 6 probe). Tano committed the fewest epm on his week 4 maintenance probe (see Figure 3, page 84). 
Types of errors per minute. Table 8 (page 85) displays Tano's performance in the type of epm committed during baseline and repeated readings. His types of epm were as follows:

Omissions. During baseline Tano committed a mean of 1.14 (range 0-5) omissions. During intervention Tano committed a mean of 0.65 (range 0-4) omissions. He committed a mean of 0.49 fewer omissions per minute during intervention than in baseline.

Additions. During baseline Tano committed a mean of 0.71 (range 0-3) additions. During intervention Tano committed a mean of 0.24 (range 0-2) additions. He committed a mean of 0.47 fewer additions per minute during intervention than in baseline.

Substitutions. During baseline Tano committed a mean of 1.71 (range 0-4) substitutions. During intervention Tano committed a mean of 1.41 (range 0-5) substitutions. He committed a mean of 0.3 fewer substitutions per minute during intervention than in baseline.

Mispronunciations. During baseline, Tano's committed a mean of 5.71 (range 38) mispronunciations. During intervention Tano committed a mean of 3.0 (range 0-6) mispronunciations. He committed a mean of 2.71 fewer mispronunciations per minute during intervention than in baseline.

Hesitations. During baseline, Tano's committed a mean of 0.29 (range 0-1) hesitations. During intervention Tano committed a mean of 0.14 (range 0-1) hesitations. He committed a mean of 0.15 fewer hesitations per minute during intervention than in baseline. 
Table 6

Mean Errors Per Minute in Comparison Sessions and Generalization Probes

\begin{tabular}{|c|c|c|c|c|}
\hline \multicolumn{2}{|c|}{ Participant } & \multicolumn{3}{|c|}{ Errors Per Minute } \\
\hline & & Baseline & Repeated Readings & Maintenance \\
\hline \multirow[t]{2}{*}{ Tano } & $\begin{array}{l}\text { Comparison } \\
\text { Scores }\end{array}$ & $\begin{array}{c}7 \\
(7-7)\end{array}$ & $\begin{array}{c}5.7 \\
(4-8)\end{array}$ & 5 \\
\hline & $\begin{array}{c}\text { Generalization } \\
\text { Probes }\end{array}$ & $\begin{array}{c}10.5 \\
(9-12)\end{array}$ & $\begin{array}{c}6.4 \\
(3-11)\end{array}$ & 6 \\
\hline \multirow[t]{2}{*}{ Luci } & $\begin{array}{l}\text { Comparison } \\
\text { Scores }\end{array}$ & $\begin{array}{c}8.67 \\
(7-11)\end{array}$ & $\begin{array}{c}5.5 \\
(2-10)\end{array}$ & 6 \\
\hline & $\begin{array}{c}\text { Generalization } \\
\text { Probes }\end{array}$ & $\begin{array}{c}7.67 \\
(4-10)\end{array}$ & $\begin{array}{l}6.25 \\
(4-9)\end{array}$ & 7 \\
\hline \multirow[t]{2}{*}{ Sandra } & $\begin{array}{l}\text { Comparison } \\
\text { Scores }\end{array}$ & $\begin{array}{c}13 \\
(11-15)\end{array}$ & $\begin{array}{c}6.6 \\
(4-9)\end{array}$ & 7 \\
\hline & $\begin{array}{c}\text { Generalization } \\
\text { Probes }\end{array}$ & $\begin{array}{l}10.6 \\
(7-14)\end{array}$ & $\begin{array}{c}7 \\
(6-10)\end{array}$ & 10 \\
\hline \multirow[t]{2}{*}{ Gema } & $\begin{array}{l}\text { Comparison } \\
\text { Scores }\end{array}$ & $\begin{array}{c}9.17 \\
(5-15)\end{array}$ & $\begin{array}{c}6.5 \\
(6-7)\end{array}$ & 2 \\
\hline & $\begin{array}{c}\text { Generalization } \\
\text { Probes }\end{array}$ & $\begin{array}{c}9.33 \\
(6-16)\end{array}$ & $\begin{array}{c}7 \\
(5-9)\end{array}$ & 5 \\
\hline \multirow[t]{2}{*}{$\begin{array}{l}\text { Group } \\
\text { Means }\end{array}$} & $\begin{array}{l}\text { Comparison } \\
\text { Scores }\end{array}$ & $\begin{array}{l}9.36 \\
(5-15)\end{array}$ & $\begin{array}{c}5.52 \\
(4-10)\end{array}$ & $\begin{array}{c}5 \\
(2-7)\end{array}$ \\
\hline & $\begin{array}{c}\text { Generalization } \\
\text { Probes }\end{array}$ & $\begin{array}{c}9.56 \\
(4-16)\end{array}$ & $\begin{array}{c}6.52 \\
(3-11)\end{array}$ & $\begin{array}{c}7 \\
(5-10)\end{array}$ \\
\hline
\end{tabular}

Note. Comparison scores and ranges are above generalization probe scores and ranges for each participant. Top numbers indicated mean number of errors read per minute. The bottom rows of numbers represent the ranges of scores. 
Table 7

Maintenance on Errors Per Minute

\begin{tabular}{lcccc}
\hline Participant & $\begin{array}{c}\text { Last Intervention } \\
\text { Score }\end{array}$ & $\begin{array}{c}\text { Week 2 } \\
\text { Probe }\end{array}$ & $\begin{array}{c}\text { Week } 4 \\
\text { Probe }\end{array}$ & $\begin{array}{c}\text { Week 6 } \\
\text { Probe }\end{array}$ \\
\hline Tano & 4 & 5 & 2 & 10 \\
& 6 & $(+1)$ & $(-2)$ & $(+6)$ \\
Luci & & 6 & 4 & 3 \\
& 6 & $(0)$ & $(-2)$ & $(-3)$ \\
Sandra & & 7 & 9 & 10 \\
& 6 & $(+1)$ & $(+3)$ & $(+4)$ \\
Gema & & 2 & 6 & 4 \\
& & $(-4)$ & $(0)$ & $(-2)$ \\
Group & 5.5 & 5 & 5.25 & 6.75 \\
& & $(-0.5)$ & $(-0.25)$ & $(+1.25)$ \\
\hline
\end{tabular}

Note. Top numbers indicate individual number of errors per minute on the last day of treatment and on 2-, 4-, and 6-week maintenance probes. The bottom row of numbers indicate the number of errors per minute more or fewer on 2-, 4- and 6-week maintenance probes as compared to the last day of intervention.

Table 9 (page 89) displays individual mean types of errors per minute in comparison sessions and in generalization probes. Tano's types of errors per minute were as follows:

Omissions. During baseline the comparison omissions score for Tano was 0.5 (range 0-1). His mean omissions score for baseline generalization was 2.0 (range 1-3). In baseline, his mean generalization score was 1.5 omissions higher than his comparison probe score. 
During intervention Tano's mean omissions score was 0.4 (range $0-2$ ). His mean omissions scores for intervention generalization was 0.4 omissions (range $0-2$ ). In intervention, his mean generalization score was equal to his comparison probe score. During maintenance, Tano's omissions comparison score was 0 omissions. His omissions score for maintenance generalization was 1 omission. In maintenance, his generalization score was 1 omission higher than his comparison probe score.

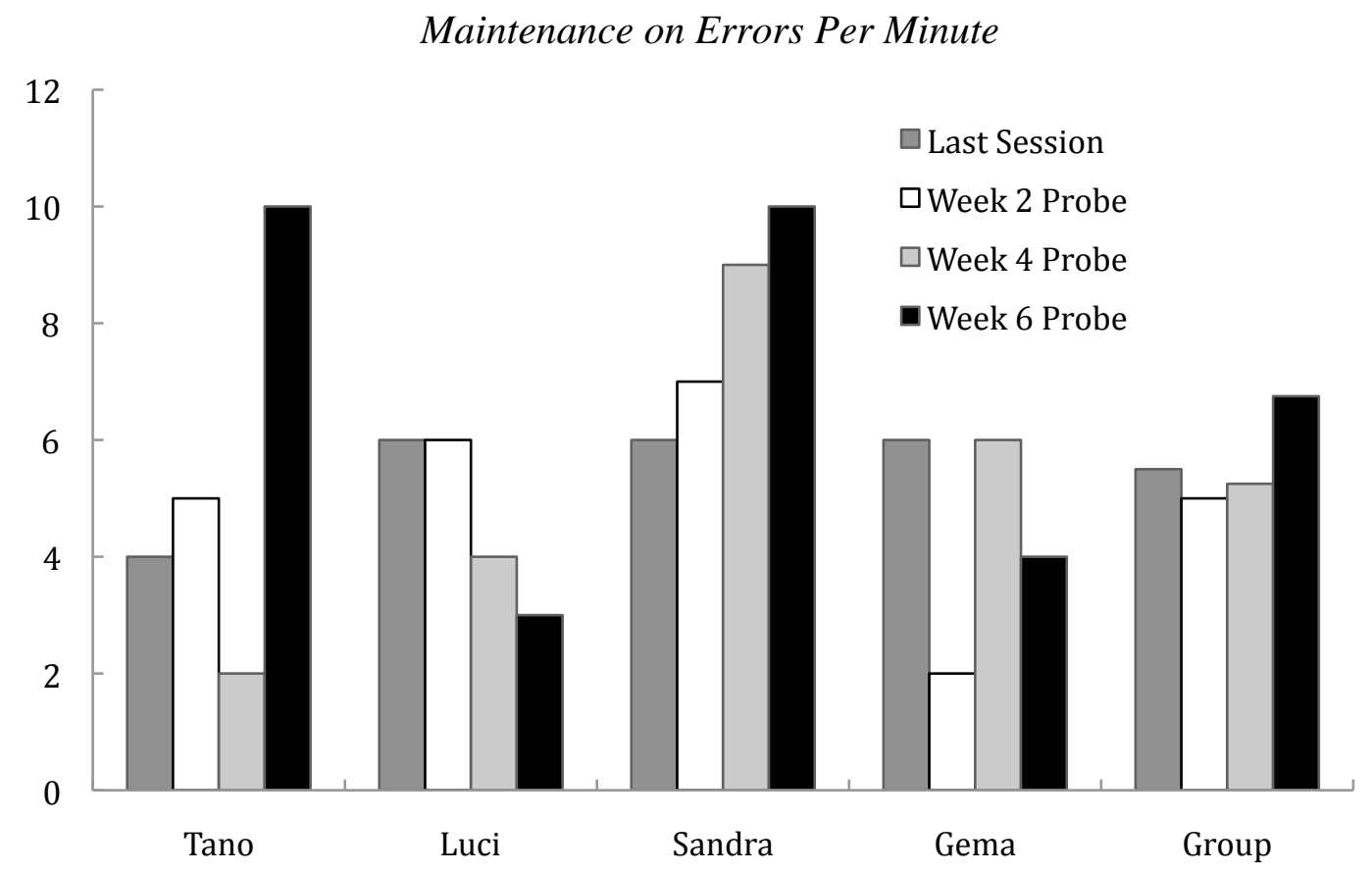

Figure 3. Epm on the last intervention session followed by epm in maintenance sessions 2-, 4-, and 6-weeks after the last intervention session.

Additions. During baseline, the comparison additions score for Tano was 0.5 additions (range 0-1). His mean additions score for baseline generalization was 0 . In baseline, his mean generalization score was 0.5 additions lower than his comparison probe score.

During intervention, Tano's mean additions score was 0.1 additions (range 0-1). His mean additions scores for intervention generalization was 0.3 additions (range $0-1$ ). 
In intervention, his mean generalization score was a mean of 0.2 additions higher than his comparison probe score.

During maintenance, Tano's epm comparison score was 1 addition. His additions score for maintenance generalization was 1 addition. In maintenance, his generalization score was equal to his comparison probe score.

Table 8

Individual Means for Types of Errors Per Minute

\begin{tabular}{|c|c|c|c|c|c|c|c|c|c|c|}
\hline \multirow[t]{3}{*}{ Participant } & \multicolumn{10}{|c|}{ Types of Errors Per Minute } \\
\hline & \multicolumn{2}{|c|}{ Omission } & \multicolumn{2}{|c|}{ Addition } & \multicolumn{2}{|c|}{ Substitution } & \multicolumn{2}{|c|}{ Mispronunciation } & \multicolumn{2}{|c|}{ Hesitation } \\
\hline & $\mathrm{BL}$ & $\mathrm{RR}$ & $\mathrm{BL}$ & RR & $\mathrm{BL}$ & RR & $\mathrm{BL}$ & $\mathrm{RR}$ & $\mathrm{BL}$ & $\mathrm{RR}$ \\
\hline \multirow[t]{2}{*}{ Tano } & 1.14 & 0.65 & 0.71 & 0.24 & 1.71 & 1.41 & 5.71 & 3.0 & 0.29 & 0.14 \\
\hline & $(0-5)$ & $(0-4)$ & $(0-3)$ & $(0-2)$ & $(0-4)$ & $(0-5)$ & $(3-8)$ & $(0-6)$ & $(0-1)$ & $(0-1)$ \\
\hline \multirow[t]{2}{*}{ Luci } & 0.42 & 0.39 & 0.25 & 0.39 & 1.5 & 2.77 & 6.5 & 1.84 & 0.58 & 0.15 \\
\hline & $(0-1)$ & $(0-3)$ & $(0-1)$ & $(0-2)$ & $(0-4)$ & $(0-9)$ & $(0-11)$ & $(0-4)$ & $(0-2)$ & $(0-1)$ \\
\hline \multirow[t]{2}{*}{ Sandra } & 0.77 & 0.8 & 0.41 & 0.35 & 2.94 & 2.2 & 5.06 & 2.65 & 0.65 & 0.45 \\
\hline & $(0-4)$ & $(0-2)$ & $(0-2)$ & $(0-2)$ & $(0-9)$ & $(0-5)$ & $(1-10)$ & $(1-6)$ & $(0-3)$ & $(0-1)$ \\
\hline \multirow[t]{2}{*}{ Gema } & 0.38 & 0.17 & 0.62 & 0.83 & 3.0 & 1.83 & 5.0 & 1.5 & 0.19 & 0.67 \\
\hline & $(0-3)$ & $(0-1)$ & $(0-2)$ & $(0-2)$ & $(1-8)$ & $(1-6)$ & $(1-14)$ & $(0-4)$ & $(0-1)$ & $(0-3)$ \\
\hline \multirow[t]{2}{*}{ Group } & 0.68 & 0.5 & 0.5 & 0.45 & 2.29 & 2.05 & 5.57 & 2.25 & 0.43 & 0.35 \\
\hline & $(0-5)$ & $(0-4)$ & $(0-3)$ & $(0-2)$ & $(0-9)$ & $(0-9)$ & $(0-14)$ & $(0-6)$ & $(0-3)$ & $(0-3)$ \\
\hline
\end{tabular}

Note. Top number indicates mean number of errors in baseline (BL) and repeated readings (RR). Bottom numbers indicate ranges. 
Substitutions. During baseline, the comparison substitutions score for Tano was 0.5 substitutions (range $0-1$ ). His mean substitutions score for baseline generalization was 1.5 (range 1-2). In baseline, his mean generalization score was 1 substitution higher than his comparison probe score.

During intervention, Tano's mean substitutions score was 2.2 (range 0-6). His mean substitutions scores for intervention generalization was 1.9 substitutions (range 05). In intervention, his mean generalization score was a mean of 0.3 substitutions higher than his comparison probe score.

During maintenance, Tano's substitutions comparison score was 1. His substitutions score for maintenance generalization was 0 substitutions. In maintenance, his generalization score was 1 substitution lower than his comparison probe score.

Mispronunciations. During baseline the comparison mispronunciations score for Tano was 5.5 (range 5-6). His mean mispronunciations score for baseline generalization was 6.5 (range 5-8). In baseline, his mean generalization score was 1 mispronunciation lower than his comparison probe score.

During intervention, Tano's mean mispronunciations score was 3.4 (range 3-6). His mean mispronunciations score for intervention generalization was 3.1 (range 3-6). In intervention, his mean generalization score was a mean of 0.3 mispronunciations lower than his comparison probe score.

During maintenance, Tano's mispronunciations comparison score was 4. His mispronunciations score for maintenance generalization was 3. In maintenance, his generalization score was 1 mispronunciation lower than his comparison probe score. 
Hesitations. During baseline, the comparison hesitations score for Tano was 0.

His mean hesitations score for baseline generalization was 0.5 hesitations (range $0-1$ ). In baseline, his mean generalization score was 0.5 hesitations higher than his comparison probe score.

During intervention, Tano's mean hesitations score was 0.2 (range 0-1). His mean hesitations scores for intervention generalization was 0.3 (range 0-2). In intervention, his mean generalization score was a mean of 0.1 hesitations higher than his comparison probe score.

During maintenance, Tano's hesitations comparison score was 0. His hesitations score for maintenance generalization was 1 . In maintenance, his generalization score was 1 hesitation higher than his comparison probe score.

Table 10 (page 90) displays Tano’s maintenance performance. Maintenance probes were taken 2-, 4-, and 6-weeks after the last intervention session. Tano's types of errors per minute on his last day of intervention and subsequent maintenance probes were as follows:

Omissions. Tano's last day intervention score was 0 omissions. He had maintenance scores of 0,1 , and 2 omissions respectively. Overall in maintenance, Tano performed between the same number of omissions as the last session (week 2 probe) and 2 omissions more than the last session (week 6 probe). Tano committed the fewest omissions on his week 2 maintenance probe.

Additions. Tano's last day intervention score was 0 additions. He had maintenance scores of 1,1 , and 0 additions respectively. Overall in maintenance, Tano performed between the same number of additions as the last session (week 6 probe) and 1 
addition more than the last session (weeks 2 and 4 probes). Tano committed the fewest additions on his week 6 maintenance probe.

Substitutions. Tano's last day intervention score was 1 substitution. He had maintenance scores of 1,0 , and 5 substitutions respectively. Overall in maintenance, Tano performed between 1 substitution fewer than the last session (week 2 probe) and 4 substitutions more than the last session (week 6 probe). Tano committed the fewest substitutions on his week 2 maintenance probe.

Mispronunciations. Tano's last day intervention score was 1 mispronunciation. He had maintenance scores of 4, 0 , and 3 mispronunciations respectively. Overall in maintenance, Tano performed between one fewer mispronunciation than the last session (week 4 probe) and 4 mispronunciations more than the last session (week 2 probe). Tano committed the fewest mispronunciations on his week 4 maintenance probe.

Hesitations. Tano's last day intervention score was 0 hesitations. He had maintenance scores of 0,0 , and 0 hesitations respectively. Overall in maintenance, Tano performed equally well in all three maintenance probes.

Luci

Number of errors per minute. Figure 2 (page 79) displays Luci's performance in the number of errors committed per minute during baseline, repeated readings, generalization probes, and maintenance probes. During baseline, Luci's mean number of epm was 9.54 (range of 7-13). When repeated readings was introduced, Luci's mean epm decreased to 5.5 epm (range 2-10). Overall, Luci's epm were lowest during the repeated readings intervention, with a decrease of $4.04 \mathrm{epm}$ from baseline. A summary of individual mean performance on the number of epm is found in Table 5 (page 80). 
Table 9

Mean Types of Errors Per Minute in Comparison Sessions and Generalization Probes.

\begin{tabular}{|c|c|c|c|c|c|c|c|c|c|c|c|c|c|c|c|c|}
\hline \multirow[t]{2}{*}{ Name } & \multirow[t]{2}{*}{ Session } & \multicolumn{3}{|c|}{ Omission } & \multicolumn{3}{|c|}{ Addition } & \multicolumn{3}{|c|}{ Substitution } & \multicolumn{3}{|c|}{ Mispronunciation } & \multicolumn{3}{|c|}{ Hesitation } \\
\hline & & BL & RR & $\mathrm{M}$ & BL & RR & $\mathrm{M}$ & BL & $\mathrm{RR}$ & $\mathrm{M}$ & BL & $\mathrm{RR}$ & $\mathrm{M}$ & BL & RR & $\mathrm{M}$ \\
\hline \multirow[t]{2}{*}{ Tano } & Comp & $\begin{array}{c}0.5 \\
(0-1)\end{array}$ & $\begin{array}{c}0.4 \\
(0-2)\end{array}$ & 0 & $\begin{array}{c}0.5 \\
(0-1)\end{array}$ & $\begin{array}{c}0.1 \\
(0-1)\end{array}$ & 1 & $\begin{array}{c}0.5 \\
(0-1)\end{array}$ & $\begin{array}{c}2.2 \\
(0-6)\end{array}$ & 1 & $\begin{array}{c}5.5 \\
(5-6)\end{array}$ & $\begin{array}{c}3.4 \\
(3-6)\end{array}$ & 4 & $\begin{array}{c}0 \\
(0)\end{array}$ & $\begin{array}{c}0.2 \\
(0-1)\end{array}$ & 0 \\
\hline & Gen & $\begin{array}{c}2.0 \\
(1-3)\end{array}$ & $\begin{array}{c}0.4 \\
(0-2)\end{array}$ & 1 & $\begin{array}{c}0 \\
(0)\end{array}$ & $\begin{array}{c}0.3 \\
(0-1)\end{array}$ & 1 & $\begin{array}{c}1.5 \\
(1-2)\end{array}$ & $\begin{array}{c}1.9 \\
(0-5)\end{array}$ & 0 & $\begin{array}{c}6.5 \\
(5-8)\end{array}$ & $\begin{array}{c}3.1 \\
(3-6)\end{array}$ & 3 & $\begin{array}{c}0.5 \\
(0-1)\end{array}$ & $\begin{array}{c}0.3 \\
(0-2)\end{array}$ & 1 \\
\hline \multirow[t]{2}{*}{ Luci } & Comp & $\begin{array}{l}0.67 \\
(0-1)\end{array}$ & $\begin{array}{l}0.63 \\
(0-3)\end{array}$ & 1 & $\begin{array}{l}0.33 \\
(0-1)\end{array}$ & $\begin{array}{l}0.25 \\
(0-1)\end{array}$ & 0 & $\begin{array}{c}1 \\
(0-3)\end{array}$ & $\begin{array}{l}2.38 \\
(1-3)\end{array}$ & 5 & $\begin{array}{c}6.0 \\
(5-10)\end{array}$ & $\begin{array}{l}1.63 \\
(0-3)\end{array}$ & 0 & $\begin{array}{l}0.67 \\
(0-1)\end{array}$ & $\begin{array}{c}0.13 \\
(0-1)\end{array}$ & 0 \\
\hline & Gen & $\begin{array}{c}0.33 \\
(0-1)\end{array}$ & $\begin{array}{c}0.13 \\
(0-1)\end{array}$ & 0 & $\begin{array}{c}0.67 \\
(0-1)\end{array}$ & $\begin{array}{c}0.25 \\
(0-1)\end{array}$ & 5 & $\begin{array}{c}1 \\
(0-2)\end{array}$ & $\begin{array}{c}3 \\
(2-5)\end{array}$ & 0 & $\begin{array}{l}4.33 \\
(2-7)\end{array}$ & $\begin{array}{l}3.38 \\
(0-6)\end{array}$ & 2 & $\begin{array}{c}0 \\
(0)\end{array}$ & $\begin{array}{l}0.25 \\
(0-1)\end{array}$ & 0 \\
\hline \multirow[t]{2}{*}{ Sandra } & Comp & $\begin{array}{c}1.2 \\
(0-2)\end{array}$ & $\begin{array}{c}0.8 \\
(0-2)\end{array}$ & 1 & $\begin{array}{c}0.6 \\
(0-1)\end{array}$ & $\begin{array}{c}0.2 \\
(0-1)\end{array}$ & 1 & $\begin{array}{c}4.6 \\
(0-7)\end{array}$ & $\begin{array}{c}2.6 \\
(2-5)\end{array}$ & 4 & $\begin{array}{c}5.2 \\
(4-7)\end{array}$ & $\begin{array}{c}4.2 \\
(2-10)\end{array}$ & 0 & $\begin{array}{c}0.2 \\
(0-1)\end{array}$ & $\begin{array}{c}0.4 \\
(0-1)\end{array}$ & 1 \\
\hline & Gen & $\begin{array}{c}1.2 \\
(0-5)\end{array}$ & $\begin{array}{c}0.4 \\
(0-1)\end{array}$ & 2 & $\begin{array}{c}0.67 \\
(0-1)\end{array}$ & $\begin{array}{c}0 \\
(0)\end{array}$ & 0 & $\begin{array}{c}3.6 \\
(0-7)\end{array}$ & $\begin{array}{c}2.2 \\
(1-4)\end{array}$ & 4 & $\begin{array}{c}4.2 \\
(2-9)\end{array}$ & $\begin{array}{c}3.4 \\
(2-5)\end{array}$ & 1 & $\begin{array}{c}1 \\
(0-2)\end{array}$ & $\begin{array}{c}1.4 \\
(0-3)\end{array}$ & 3 \\
\hline \multirow[t]{2}{*}{ Gema } & Comp & $\begin{array}{c}1.5 \\
(0-4)\end{array}$ & $\begin{array}{c}0.5 \\
(0-1)\end{array}$ & 0 & $\begin{array}{c}0.33 \\
(0-1)\end{array}$ & $\begin{array}{c}1 \\
(1-1)\end{array}$ & 0 & $\begin{array}{l}1.83 \\
(0-4)\end{array}$ & $\begin{array}{c}3.5 \\
(1-6)\end{array}$ & 0 & $\begin{array}{c}5.33 \\
(2-11)\end{array}$ & $\begin{array}{c}1.5 \\
(0-3)\end{array}$ & 0 & $\begin{array}{c}0 \\
(0)\end{array}$ & $\begin{array}{c}0 \\
(0)\end{array}$ & 2 \\
\hline & Gen & $\begin{array}{l}0.83 \\
(0-2)\end{array}$ & $\begin{array}{c}0.5 \\
(0-1)\end{array}$ & 0 & $\begin{array}{l}0.67 \\
(0-1)\end{array}$ & $\begin{array}{c}0.5 \\
(0-1)\end{array}$ & 0 & $\begin{array}{l}3.83 \\
(1-6)\end{array}$ & $\begin{array}{c}3 \\
(1-4)\end{array}$ & 1 & $\begin{array}{c}3.67 \\
(0-14)\end{array}$ & $\begin{array}{c}2 \\
(1-3)\end{array}$ & 4 & $\begin{array}{l}0.33 \\
(0-1)\end{array}$ & $\begin{array}{c}1 \\
(0-2)\end{array}$ & 0 \\
\hline \multirow[t]{2}{*}{ Group } & Comp & $\begin{array}{l}1.13 \\
(0-4)\end{array}$ & $\begin{array}{c}0.48 \\
(0-3)\end{array}$ & $\begin{array}{c}0.5 \\
(0-1)\end{array}$ & $\begin{array}{c}0.44 \\
(0-1)\end{array}$ & $\begin{array}{c}0.21 \\
(0-1)\end{array}$ & $\begin{array}{c}0.5 \\
(0-1)\end{array}$ & $\begin{array}{l}2.38 \\
(0-7)\end{array}$ & $\begin{array}{l}2.10 \\
(0-6)\end{array}$ & $\begin{array}{c}2.5 \\
(0-5)\end{array}$ & $\begin{array}{c}5.44 \\
(0-11)\end{array}$ & $\begin{array}{c}3.10 \\
(0-10)\end{array}$ & $\begin{array}{c}1 \\
(0-4)\end{array}$ & $\begin{array}{l}0.19 \\
(0-1)\end{array}$ & $\begin{array}{c}0.17 \\
(0-1)\end{array}$ & $\begin{array}{l}0.75 \\
(0-2)\end{array}$ \\
\hline & Gen & $\begin{array}{c}1.0 \\
(0-5)\end{array}$ & $\begin{array}{c}0.31 \\
(0-2)\end{array}$ & $\begin{array}{c}0.75 \\
(0-2)\end{array}$ & $\begin{array}{c}0.56 \\
(0-1)\end{array}$ & $\begin{array}{c}0.21 \\
(0-1)\end{array}$ & $\begin{array}{c}1.5 \\
(0-5)\end{array}$ & $\begin{array}{l}2.94 \\
(0-7)\end{array}$ & $\begin{array}{l}2.07 \\
(0-5)\end{array}$ & $\begin{array}{l}1.25 \\
(0-4)\end{array}$ & $\begin{array}{c}3.13 \\
(0-14)\end{array}$ & $\begin{array}{l}2.69 \\
(0-6)\end{array}$ & $\begin{array}{c}2.5 \\
(1-4)\end{array}$ & $\begin{array}{c}0.5 \\
(0-2)\end{array}$ & $\begin{array}{c}0.48 \\
(0-3)\end{array}$ & $\begin{array}{c}1 \\
(0-3)\end{array}$ \\
\hline
\end{tabular}

Note. Top number indicates mean number of epm in baseline (BL), repeated readings (RR), and maintenance (M) comparison (Comp) and generalization (Gen), bottom indicate ranges. 
Table 10

Maintenance Performance Types of Errors Per Minute

\begin{tabular}{|c|c|c|c|c|c|c|}
\hline \multirow[t]{2}{*}{ Participant } & & \multicolumn{5}{|c|}{ Types of Errors Per Minute } \\
\hline & & Omission & Addition & Substitution & Mispronunciation & Hesitation \\
\hline \multirow[t]{7}{*}{ Tano } & Last RR & 0 & 0 & 1 & 1 & 0 \\
\hline & 2-weeks & 0 & 1 & 1 & 4 & 0 \\
\hline & & $(0)$ & $(+1)$ & (0) & $(+3)$ & (0) \\
\hline & 4-weeks & 1 & 1 & 0 & 0 & 0 \\
\hline & & $(+1)$ & $(+1)$ & $(-1)$ & $(-1)$ & (0) \\
\hline & 6-weeks & 2 & 0 & 5 & 3 & 0 \\
\hline & & $(+2)$ & (0) & $(+4)$ & $(=2)$ & $(0)$ \\
\hline \multirow[t]{7}{*}{ Luci } & Last RR & 0 & 0 & 4 & 2 & 0 \\
\hline & 2-weeks & 1 & 0 & 5 & 0 & 0 \\
\hline & & $(+1)$ & (0) & $(+4)$ & $(-2)$ & (0) \\
\hline & 4-weeks & 1 & 0 & 1 & 1 & 0 \\
\hline & & $(+1)$ & (0) & $(-3)$ & $(-1)$ & (0) \\
\hline & 6-weeks & 0 & 0 & 1 & 2 & 0 \\
\hline & & (0) & (0) & $(-3)$ & (0) & (0) \\
\hline \multirow[t]{7}{*}{ Sandra } & Last RR & 2 & 0 & 0 & 4 & 0 \\
\hline & 2-weeks & 1 & 1 & 4 & 0 & 1 \\
\hline & & $(-1)$ & $(+1)$ & $(+4)$ & $(-4)$ & $(+1)$ \\
\hline & 4-weeks & 0 & 0 & 2 & 2 & 5 \\
\hline & & $(-2)$ & (0) & $(+2)$ & $(-2)$ & $(+5)$ \\
\hline & 6-weeks & 1 & 0 & 3 & 5 & 1 \\
\hline & & $(-1)$ & (0) & $(+3)$ & $(+1)$ & $(+1)$ \\
\hline \multirow[t]{7}{*}{ Gema } & Last RR & 1 & 1 & 1 & 3 & 0 \\
\hline & 2-weeks & 0 & 0 & 0 & 0 & 2 \\
\hline & & $(-1)$ & $(-1)$ & $(-1)$ & $(-3)$ & $(+2)$ \\
\hline & 4-weeks & 0 & 0 & 1 & 3 & 2 \\
\hline & & $(-1)$ & $(-1)$ & (0) & (0) & $(+2)$ \\
\hline & 6-weeks & 0 & 0 & 0 & 4 & 0 \\
\hline & & $(-1)$ & $(-1)$ & $(-1)$ & $(-1)$ & (0) \\
\hline Group & Last RR & 0.75 & 0.25 & 1.5 & 2.5 & 0 \\
\hline \multirow[t]{6}{*}{ Means } & 2-weeks & 0.5 & 0.5 & 2.5 & 1.0 & 0.75 \\
\hline & & $(-0.25)$ & $(+0.25)$ & $(+1.0)$ & $(-1.5)$ & $(+0.75)$ \\
\hline & 4-weeks & 0.5 & 0.25 & 1.0 & 1.5 & 1.75 \\
\hline & & $(-0.25)$ & (0) & $(-0.5)$ & $(-1.0)$ & $(+1.75)$ \\
\hline & 6-weeks & 0.75 & 0 & 2.25 & 3.5 & 0.25 \\
\hline & & $(0)$ & $(-0.25)$ & $(+0.75)$ & $(+1.0)$ & $(+0.25)$ \\
\hline
\end{tabular}

Note. Top numbers indicate individual number of epm on the last day of treatment. Bottom numbers indicate the number of epm more or fewer on 2-, 4-, and 6-week maintenance probes as compared to the last day of intervention. 
Table 6 (page 82) displays individual mean epm in sessions and in comparison sessions and generalization probes. During baseline the comparison epm score for Luci was 8.67 epm (range 7-11). Her mean epm score for baseline generalization was 7.67 epm (range 4-10). In baseline, her mean generalization score was 1epm lower than her comparison probe score.

During intervention Luci's mean epm score was 5.5 epm (range 2-10). Her mean epm scores for intervention generalization was 6.25 epm (range 4-9). In intervention, her mean generalization score was a mean of 0.75 epm more than her comparison probe score.

During maintenance, Luci's epm comparison score was 6 epm. Her epm score for maintenance generalization was $7 \mathrm{epm}$. In maintenance, her generalization score was lepm more than her comparison probe score.

In comparing baseline, intervention and maintenance epm generalization scores, Luci committed an average of 1.42 fewer epm during intervention generalization probes than baseline generalization probes. Luci's epm were lowest on her intervention generalization probes with a mean of $6.25 \mathrm{epm}$.

Table 7 (page 83) displays Luci's maintenance performance. On Luci’s last day intervention she committed $6 \mathrm{epm}$. She had maintenance scores of 6,4 , and 3 respectively. Overall in maintenance, Luci performed between 3 epm fewer than the last session (week 6 probe) and the same number of epm as the last session (week 2 probe). Luci committed the fewest epm on her week 6 maintenance probe (see Figure 3, page $84)$. 
Types of errors per minute. Table 8 (page 85) displays Luci's performance in the type of errors committed per minute during baseline and repeated readings. Her types of epm were as follows:

Omissions. During baseline Luci committed a mean of 0.42 (range 0-1) omissions. During intervention Luci committed a mean of 0.39 (range 0-3) omissions. She committed a mean of 0.01 fewer omissions per minute during intervention than in baseline.

Additions. During baseline Luci committed a mean of 0.25 (range 0-1) additions. During intervention Luci committed a mean of 0.39 (range 0-2) additions. She committed a mean of 0.14 more additions per minute during intervention than in baseline.

Substitutions. During baseline, Luci committed a mean of 1.5 (range 0-4) substitutions. During intervention Luci committed a mean of 2.77 (range 0-9) substitutions. She committed a mean of 1.27 more substitutions per minute during intervention than in baseline.

Mispronunciations. During baseline, Luci committed a mean of 6.5 (range 3-11) mispronunciations. During intervention Luci committed a mean of 1.84 (range 0-4) mispronunciations. She committed a mean of 4.66 fewer mispronunciations per minute during intervention than in baseline.

Hesitations. During baseline, Luci committed a mean of 0.58 (range 0-2) hesitations. During intervention Luci committed a mean of 0.15 (range 0-1) hesitations. She committed a mean of 0.43 fewer hesitations during intervention than in baseline. 
Table 9 (page 89) displays individual mean types of errors per minute in comparison sessions and in generalization probes. Luci's types of errors per minute were as follows:

Omissions. During baseline the comparison omissions score for Luci was 0.67 (range 0-1). Her mean omissions score for baseline generalization was 0.33 (range 0-1). In baseline, her mean generalization score was 0.34 omissions lower than her comparison probe score.

During intervention Luci's mean omissions score was 0.63 (range 0-3). Her mean omissions scores for intervention generalization was 0.13 (range 0-1). In intervention, her mean generalization score was a mean of 0.5 omissions lower than her comparison probe score.

During maintenance, Luci's omission comparison score was 1. Her omissions score for maintenance generalization was 0 . In maintenance, her generalization score was 1 omission lower than her comparison probe score.

Additions. During baseline the comparison mean additions score for Luci was 0.33 additions (range $0-1$ ). Her mean additions score for baseline generalization was 0.67 (range 0-1). In baseline, her mean generalization score was 0.34 additions higher than her mean comparison probe score.

During intervention Luci's mean additions score was 0.25 (range 0-1). Her mean additions scores for intervention generalization was 0.25 (range $0-1)$. In intervention, her mean generalization score was equal to her mean comparison probe score. 
During maintenance, Luci's mean additions comparison score was 0. Her mean additions score for maintenance generalization was 5. In maintenance, her mean generalization score was 5 additions higher than her mean comparison probe score.

Substitutions. During baseline the mean comparison substitutions score for Luci was 1 (range $0-3$ ). Her mean substitutions score for baseline generalization was 1 (range 0-2). In baseline, her mean generalization score was equal to her comparison probe score.

During intervention Luci's mean substitutions score was 2.38 (range 1-3). Her mean substitution scores for intervention generalization was 3.0 (range 2-5). In intervention, her mean generalization score was 0.62 substitutions higher than her mean comparison probe score.

During maintenance, Luci's substitutions comparison score was 5. Her substitutions score for maintenance generalization was 0 . In maintenance, her generalization score was 5 substitutions lower than her comparison probe score.

Mispronunciations. During baseline the mean comparison mispronunciations score for Luci was 6 (range 5-10). Her mean mispronunciations score for baseline generalization was 4.33 (range 2-7). In baseline, her mean generalization score was 1.67 mispronunciations lower than her comparison probe score.

During intervention Luci's mean mispronunciations score was 1.63 (range 0-3). Her mean mispronunciation scores for intervention generalization was 3.38 (range 0-6). In intervention, her mean generalization score was 1.75 mispronunciations higher than her mean comparison probe score. 
During maintenance, Luci's mispronunciation comparison score was 0. Her mispronunciations score for maintenance generalization was 2. In maintenance, her generalization score was 2 mispronunciations higher than her comparison probe score.

Hesitations. During baseline the mean comparison hesitations score for Luci was 0.67 (range 0-1). Her mean hesitations score for baseline generalization was 0 . In baseline, her mean generalization score was 0.67 hesitations lower than her comparison probe score.

During intervention Luci's mean hesitations score was 0.13 (range 0-1). Her mean hesitations scores for intervention generalization was 0.25 (range $0-1$ ). In intervention, her mean generalization score was 0.12 hesitations higher than her mean comparison probe score.

During maintenance, Luci's hesitations comparison score was 0. Her hesitations score for maintenance generalization was 0 . In maintenance, her generalization score was equal to her comparison probe score.

Table 10 (page 90) displays Luci's maintenance performance. Maintenance probes were taken 2-, 4-, and 6-weeks after the last intervention session. Luci's types of epm on her last day of intervention and subsequent maintenance probes were as follows:

Omissions. Luci's last day of intervention score was 0 omissions. She had maintenance scores of 1,1 , and 0 omissions respectively. Overall in maintenance, Luci performed between 1 omission fewer than the last session (weeks 2 and 4 probes) and the same number of omissions as the last session (week 6 probe). Luci committed the fewest omissions on her week 6 maintenance probe. 
Additions. Luci's last day intervention score was 0 additions. She had maintenance scores of 0,0 , and 0 additions respectively. Overall in maintenance, Luci performed equally well in all three maintenance probes.

Substitutions. Luci's last day intervention score was 4 substitutions. She had maintenance scores of 5,1, and 1 substitutions respectively. Overall in maintenance, Luci performed between 3 substitutions fewer than the last session (weeks 4 and 6 probes) and 1 substitution more than the last session (week 2 probe). Luci committed the fewest substitutions on her weeks 4 and 6 maintenance probes.

Mispronunciations. Luci's last day intervention score was 2 mispronunciations. She had maintenance scores of 0,1 , and 2 mispronunciations respectively. Overall in maintenance, Luci performed between the same number of mispronunciations as the last session (week 6 probe) and 2 mispronunciations fewer than the last session (week 2 probe). Luci committed the fewest mispronunciations on her week 2 maintenance probe.

Hesitations. Luci's last day intervention score was 0 hesitations. She had maintenance scores of 0,0 , and 0 hesitations respectively. Overall in maintenance, Luci performed equally well in all three maintenance probes.

\section{Sandra}

Number of errors per minute. Figure 2 (page 79) displays Sandra's performance in the number of errors committed per minute during baseline, repeated readings, generalization probes, and maintenance probes. During baseline, Sandra's mean number of epm was $9.77 \mathrm{epm}$, (range 5-18). When repeated readings was introduced, Sandra's mean epm decreased to 6.27 epm (range 3-9). Overall, Sandra's epm were lowest during the repeated readings intervention, with a mean decrease of 3.5 epm from baseline. A 
summary of individual mean performance on the number of errors per minute can be found in Table 5 (page 80).

Table 6 (page 82) displays individual mean epm in comparison sessions and in generalization probes. During baseline the comparison epm score for Sandra was 13 epm (range 11-15). Her mean epm score for baseline generalization was 10.6 epm (range 714). In baseline, her mean generalization score was 2.4 fewer epm than her comparison probe score.

During intervention Sandra's mean epm score was 6.6 epm (range 4-9). Her mean epm scores for intervention generalization was 7 epm (range 6-10). In intervention, her mean generalization score was 0.4 epm more than her comparison probe score.

During maintenance, Sandra's epm comparison score was 7 epm. Her epm score for maintenance generalization was $10 \mathrm{epm}$. In maintenance, her generalization score was 3 epm more than her comparison probe score.

In comparing baseline, intervention, and maintenance epm generalization scores, Sandra committed an average of 3.6 fewer epm during intervention generalization probes than baseline generalization probes. Sandra's epm were lowest on her intervention generalization probe with a mean of $7 \mathrm{epm}$.

Table 7 (page 83) displays Sandra's maintenance performance. Sandra's epm score on her last day of intervention was $6 \mathrm{epm}$. She had maintenance scores of 7, 9, and 10 epm respectively. Overall in maintenance, Sandra performed between 1 epm more than the last session (week 2 probe) and 4 epm more than the last session (week 6 probe). Sandra committed the fewest epm on her week 2 maintenance probe (see Figure 3, page 84). 
Types of errors per minute. Table 8 (page 85) displays Sandra's performance in the type of errors committed per minute during baseline and repeated readings. Her types of epm were as follows:

Omissions. During baseline Sandra committed a mean of 0.77 (range 0-4) omissions. During intervention Sandra committed a mean of 0.8 (range 0-2) omissions. She committed a mean of 0.03 more omissions per minute during intervention than in baseline.

Additions. During baseline Sandra committed a mean of 0.41 (range 0-2) additions. During intervention Sandra committed a mean of 0.35 (range 0-2) additions. She committed a mean of 0.06 fewer additions per minute during intervention than in baseline.

Substitutions. During baseline Sandra committed a mean of 2.94 (range 0-9) substitutions. During intervention Sandra committed a mean of 2.2 (range 0-5) substitutions. She committed a mean of 0.74 fewer substitutions per minute during intervention than in baseline.

Mispronunciations. During baseline Sandra committed a mean of 5.06 (range 110) mispronunciations. During intervention Sandra committed a mean of 2.65 (range 1-6) mispronunciations. She committed a mean of 2.41 fewer omissions per minute during intervention than in baseline.

Hesitations. During baseline Sandra committed a mean of 0.65 (range 0-3) hesitations. During intervention Sandra committed a mean of 0.45 (range 0-1) hesitations. She committed a mean of 0.2 fewer omissions per minute during intervention than in baseline. 
Table 9 (page 89) displays individual mean types of errors per minute in comparison sessions and in generalization probes. Sandra's types of errors per minute were as follows:

Omissions. During baseline the mean comparison omissions score for Sandra was 1.2 (range 0-2). Her mean omissions score for baseline generalization was 1.2 (range 05). In baseline, her mean generalization score was equal to her mean comparison probe score.

During intervention Sandra's mean omissions score was 0.8 (range 0-2). Her mean omissions scores for intervention generalization was 0.4 (range $0-1)$. In intervention, her mean generalization score was 0.4 omissions lower than her mean comparison probe score.

During maintenance, Sandra's comparison score was 1 omission. Her omission score for maintenance generalization was 2. In maintenance, her generalization score was 1 omission higher than her comparison probe score.

Additions. During baseline the mean comparison additions score for Sandra was 0.6 (range 0-1). Her mean additions score for baseline generalization was 0.67 (range 01). In baseline, her mean generalization score was 0.7 additions higher than her mean comparison probe score.

During intervention Sandra's mean additions score was 0.2 (range 0-1). Her mean additions scores for intervention generalization was 0 . In intervention, her mean generalization score was 0.2 additions lower than her mean comparison probe score. 
During maintenance, Sandra's comparison score was 1addition. Her additions score for maintenance generalization was 0 . In maintenance, her generalization score was 1 addition lower than her comparison probe score.

Substitutions. During baseline the mean comparison substitutions score for Sandra was 4.6 (range 0-7). Her mean substitutions score for baseline generalization was 3.6 (range 0-7). In baseline, her mean generalization score was 1.0 substitution lower than her mean comparison probe score.

During intervention Sandra's mean substitutions score was 2.6 (range 2-5). Her mean substitutions scores for intervention generalization was 2.2 (range 1-4). In intervention, her mean generalization score was 0.4 substitutions lower than her mean comparison probe score.

During maintenance, Sandra's comparison score was 4 substitutions. Her substitutions score for maintenance generalization was 4. In maintenance, her generalization score was equal to her comparison probe score.

Mispronunciation. During baseline the mean comparison mispronunciations score for Sandra was 5.2 (range 4-7). Her mean mispronunciations score for baseline generalization was 4.2 (range 2-9). In baseline, her mean generalization score was 3.5 mispronunciations lower than her mean comparison probe score.

During intervention Sandra's mean mispronunciations score was 4.2 (range 2-10). Her mean mispronunciation score for intervention generalization was 3.4 (range 2-5). In intervention, her mean generalization score was a mean of 0.8 mispronunciations lower than her comparison probe score. 
During maintenance, Sandra's comparison score was 0 mispronunciations. Her mispronunciations score for maintenance generalization was 1. In maintenance, her generalization score was 1 mispronunciation higher than her comparison probe score.

Hesitation. During baseline the comparison hesitations score for Sandra was 0.2 (range 0-1). Her mean hesitations score for baseline generalization was 1.0 hesitation (range 0-2). In baseline, her mean generalization score was 0.8 hesitations higher than her mean comparison probe score.

During intervention Sandra's mean hesitations score was 0.4 (range 0-1). Her mean hesitations scores for intervention generalization was 1.4 (range 0-3). In intervention, her mean generalization score was 1 hesitation higher than her comparison probe score.

During maintenance, Sandra's comparison score was 1 hesitation. Her hesitations score for maintenance generalization was 3. In maintenance, her generalization score was 2 hesitations higher than her comparison probe score.

Table 10 (page 90) displays Sandra's maintenance performance. Maintenance probes were taken 2-, 4-, and 6-weeks after the last intervention session. Sandra's types of epm on her last day of intervention and subsequent maintenance probes were as follows:

Omissions. Sandra's last day intervention score was 2 omissions. She had maintenance scores of 1, 0, and 1 omission respectively. Overall in maintenance, Sandra performed between 1 omission fewer than the last session (weeks 2 and 6 probes) and 2 omissions fewer than the last session (week 4 probe). Sandra committed the fewest omissions on her week 4 maintenance probe. 
Additions. Sandra's last day intervention score was 0 additions. She had maintenance scores of 1, 0, and 0 additions respectively. Overall in maintenance, Sandra performed between the same number of additions as the last session (weeks 4 and 6 probes) and 1 addition more than the last session (week 2 probe). Sandra committed the fewest omissions on her weeks 4 and 6 maintenance probes.

Substitutions. Sandra's last day intervention score was 0 substitutions. She had maintenance scores of 4, 2, and 3 substitutions respectively. Overall in maintenance, Sandra performed between 2 substitutions more than the last session (week 4 probe) and 4 substitutions more than the last session (week 2 probe). Sandra committed the fewest substitutions on her week 4 maintenance probe.

Mispronunciations. Sandra's last day intervention score was 4 mispronunciations. She had maintenance scores of 0,2 , and 5 mispronunciations respectively. Overall in maintenance, Sandra performed between 4 mispronunciations fewer than the last session (week 2 probe) and 1 mispronunciation more than the last session (week 6 probe). Sandra committed the fewest mispronunciations on her week 2 maintenance probe (see figure 7).

Hesitations. Sandra's last day intervention score was 0 hesitations. She had maintenance scores of 1,5 , and 1 hesitations respectively. Overall in maintenance, Sandra performed between 1 hesitation more than the last session (weeks 2 and 6 probes) and 5 hesitations more than the last session (week 4 probe). Sandra committed the fewest hesitations on her weeks 2 and 6 maintenance probes.

\section{Gema}

Number of errors per minute. Figure 2 (page 79) displays Gema's performance in the number of epm committed during baseline, repeated readings, generalization probes, 
and maintenance probes. During baseline, Gema's mean number of epm was 8.95 epm (range 5-15). When repeated readings was introduced, Gema's mean epm decreased to 5.29 epm (range 1-7). Overall, Gema's epm were lowest during the repeated readings intervention, with a decrease of 3.66 epm from baseline. A summary of individual mean performance on the number of errors per minute can be found in Table 5 (page 80).

Table 6 (page 82) displays individual mean epm in comparison sessions and in generalization probes. During baseline the mean comparison epm score for Gema was 9.17 epm (range 5-15). Her mean epm score for baseline generalization was 9.33 epm (range 6-16). In baseline, her mean generalization score was 0.16 epm more than her comparison probe score.

During intervention Gema's mean epm score was 6.5 epm (range 6-7). Her mean epm scores for intervention generalization was 7 epm (range 5-9). In intervention, her mean generalization score was 0.5 epm more than her comparison probe score.

During maintenance, Gema's epm comparison score was 2 epm. Her epm score for maintenance generalization was 5 epm. In maintenance, her generalization score was 3 epm more than her comparison probe score.

In comparing baseline, intervention and maintenance epm generalization scores, Gema committed a mean of 2.33 fewer epm during intervention generalization probes than baseline generalization probes. Gema's epm were lowest on her maintenance generalization probe with 5 epm.

Table 7 (page 83) displays Gema's maintenance performance. On Gema’s epm score on her last day of intervention was $6 \mathrm{epm}$. She had maintenance scores of 2, 6, and 4 epm respectively. Overall in maintenance, Sandra performed between 4 epm fewer than 
the last session (week 2 probe) and the same number of epm as the last session (week 4 probe). Gema committed the fewest epm on her week 2 maintenance probe (see Figure 3, page 84).

Types of errors per minute. Table 8 (page 85) displays Gema's performance in the type of errors committed per minute during baseline and repeated readings. Her types of epm were as follows:

Omissions. During baseline Gema's committed a mean of 0.38 (range 0-3) omissions. During intervention Gema committed a mean of 0.17 (range 0-1) omissions. She committed a mean of 0.21 fewer omissions per minute during intervention than in baseline.

Additions. During baseline Gema committed a mean of 0.62 (range 0-2) additions. During intervention Gema committed a mean of 0.83 (range 0-2) additions. She committed a mean of 0.21 more additions per minute during intervention than in baseline.

Substitutions. During baseline Gema committed a mean of 3.0 (range 1-8) substitutions. During intervention Gema committed a mean of 1.83 (range 1-6) substitutions. She committed a mean of 1.17 fewer substitutions per minute during intervention than in baseline.

Mispronunciations. During baseline Gema committed a mean of 5.0 (range 1-14) mispronunciations. During intervention Gema committed a mean of 1.5 (range 0-4) mispronunciations. She committed a mean of 3.5 fewer mispronunciations per minute during intervention than in baseline. 
Hesitations. During baseline Gema's committed a mean of 0.19 (range 0-1)

hesitations. During intervention Gema committed a mean of 0.67 (range 0-3) hesitations. She committed 0.48 more hesitations during intervention than in baseline.

Table 9 (page 89) displays individual mean types of errors per minute in comparison sessions and in generalization probes. Gema's types of epm were as follows:

Omissions. During baseline the comparison omissions score for Gema was 1.5 (range 0-4). Her mean omissions score for baseline generalization was 0.83 (range $0-2$ ). In baseline, her mean generalization score was 0.67 omissions lower than her comparison probe score.

During intervention Gema's mean omissions score was 0.5 (range 0-1). Her mean omissions scores for intervention generalization was 0.5 (range 0-1). In intervention, her mean generalization score was equal to her comparison probe score.

During maintenance, Gema's epm comparison score was 0. Her omissions score for maintenance generalization was 0 . In maintenance, her generalization score equal to her comparison probe score.

Additions. During baseline, the comparison additions score for Gema was 0.33 (range 0-1). Her mean additions score for baseline generalization was 0.67 (range $0-1$ ). In baseline, her mean generalization score was 0.34 additions higher than her mean comparison probe score.

During intervention Gema's mean additions score was 1 (range 1-1). Her mean additions score for intervention generalization was 0.5 (range $0-1)$. In intervention, her mean generalization score was 0.5 additions higher than her mean comparison probe score. 
During maintenance, Gema's additions comparison score was 0. Her additions score for maintenance generalization was 0 . In maintenance, her generalization score was equal to her comparison probe score.

Substitutions. During baseline the comparison substitutions score for Gema was 1.83 (range 0-4). Her mean substitutions score for baseline generalization was 3.83 (range 1-6). In baseline, her mean generalization score was 2 substitutions higher than her mean comparison probe score.

During intervention Gema's mean substitutions score was 3.5 (range 1-6). Her mean substitutions scores for intervention generalization was 3.0 (range 1-4). In intervention, her mean generalization score was 0.5 substitutions lower than her mean comparison probe score.

During maintenance, Gema's substitutions comparison score was 0. Her substitutions score for maintenance generalization was 1 . In maintenance, her generalization score was 1 substitution higher than her comparison probe score.

Mispronunciations. During baseline the mean comparison mispronunciations score for Gema was 5.33 (range 2-11). Her mean mispronunciations score for baseline generalization was 3.67 (range 0-14). In baseline, her mean generalization score was 1.66 mispronunciations lower than her mean comparison probe score.

During intervention Gema's mean mispronunciations score was 1.5 (range 0-3). Her mean mispronunciations scores for intervention generalization was 2.0 (range 1-3). In intervention, her mean generalization score was 0.5 mispronunciations higher than her mean comparison probe score. 
During maintenance, Gema's mispronunciations comparison score was 0. Her mispronunciations score for maintenance generalization was 4. In maintenance, her generalization score was 4 mispronunciations higher than her comparison probe score.

Hesitation. During baseline the mean comparison hesitations score for Gema was 0. Her mean hesitations score for baseline generalization was 0.33 (range 0-1). In baseline, her mean generalization score was 0.33 hesitations higher than her mean comparison probe score.

During intervention, Gema's mean hesitations score was 0. Her mean hesitations scores for intervention generalization was 1 (range 0-2). In intervention, her mean generalization score was 1 hesitation higher than her mean comparison probe score.

During maintenance, Gema's hesitations comparison score was 2. Her hesitations score for maintenance generalization was 0 . In maintenance, her generalization score was 2 hesitations higher than her comparison probe score.

Table 10 (page 90) displays Gema's maintenance performance. Gema’s types of epm on her last day of intervention and subsequent maintenance probes were as follows:

Omissions. Gema's last day intervention score was 1 omission. She had maintenance scores of 0,0 , and 0 omissions respectively. Overall in maintenance, Gema performed equally well on all of her maintenance probes.

Additions. Gema's last day intervention score was 1 addition. She had maintenance scores of 0,0 , and 0 additions respectively. Overall in maintenance, Gema performed equally well on all of her maintenance probes.

Substitutions. Gema's last day intervention score was 1 substitution. She had maintenance scores of 0,1 , and 0 substitutions respectively. Overall in maintenance, 
Gema performed between 1 substitution fewer than the last session (weeks 2 and 6 probes) and an equal number of substitutions as the last session (week 4 probe). Gema committed the fewest substitutions on weeks 2 and 6 maintenance probes.

Mispronunciations. Gema's last day intervention score was 3 mispronunciations. She had maintenance scores of 0,3 , and 4 mispronunciations respectively. Overall in maintenance, Gema performed between 3 mispronunciations fewer than the last session (week 2 probe) and 1 mispronunciation more than the last session (week 6 probe). Gema committed the fewest mispronunciations on her week 2 maintenance probe.

Hesitations. Gema's last day intervention score was 0 hesitations. She had maintenance scores of 2, 2, and 0 hesitations respectively. Overall in maintenance, Gema performed between the same number of hesitations as the last session (week 6 probe) and 2 hesitations more than the last session (weeks 2 and 4 probes). Gema committed the fewest hesitations on her week 6 maintenance probe.

\section{Group Means for Number and Types of Errors Per Minute}

Table 5 (page 80) displays the group means for the number of epm during baseline and repeated readings. During baseline, the mean number of epm was 9.21 (range 4-18). When repeated readings was introduced, the mean number of errors per minute decreased to 5.52 (range 1-11). Overall, the mean number of epm was lowest during the repeated readings intervention, with a mean decrease of 3.69 epm from baseline.

Table 6 (page 82) displays the group means for epm in comparison sessions and in generalization probes. During baseline the mean group comparison epm score was 9.36 epm (range 5-15). The mean epm score for baseline generalization was $9.56 \mathrm{epm}$ (range 
4-16). In baseline, the group mean generalization score was 0.2 epm higher than the comparison probe score.

During intervention the group mean epm comparison score was 5.52 epm (range 4-10). The mean epm score for intervention generalization was $6.52 \mathrm{epm}$ (range 3-11). In intervention, the mean group generalization score was 1 epm higher than the comparison probe score.

During maintenance, the group epm comparison score was 5 (range 2-7). The epm score for maintenance generalization was 7 (range 5-10). In maintenance, the group mean generalization score was $2 \mathrm{epm}$ higher than the mean comparison probe score.

In comparing baseline, intervention, and maintenance epm generalization scores, the group committed a mean of 3.04 more epm during intervention generalization probes than baseline generalization probes. The group mean epm was lowest on the mean intervention generalization probes with $6.52 \mathrm{epm}$.

Table 7 (page 83) displays the group mean maintenance performance scores for epm. The group mean score on the last day of intervention was 5.5 epm (range 4-6). The group had mean maintenance scores of 5 (range 2-7), 5.25 (range 2-6), and 6.75 (range 310) epm respectively. Overall in maintenance, the group performed between a mean of 0.5 epm fewer than the last session (week 2 probe) and $1.25 \mathrm{epm}$ more than the last session (week 6 probe). The group committed the fewest epm on the week 2 maintenance probe (see Figure 3, page 84).

Table 8 (page 85) displays group means for the type of epm during baseline and repeated readings. The group means for types of epm were as follows: 
Omissions. During baseline, the mean number of omissions was 0.68 (range 0-5). During intervention the mean number of omissions was 0.5 (range0-4). In intervention there was a mean decrease of 0.18 omissions per minute from baseline.

Additions. During baseline, the mean number of additions was 0.5 (range 0-3). During intervention the mean number of additions was 0.45 (range 0-2). In intervention there was a mean decrease of 0.05 additions per minute from baseline.

Substitutions. During baseline, the mean number of substitutions was 2.29 (range 0-9). During intervention the mean number of omissions was 2.05 (range 0-9). In intervention there was a mean decrease of 0.24 substitutions per minute from baseline.

Mispronunciations. During baseline, the mean number of mispronunciations was 5.57 (range 0-14). During intervention the mean number of mispronunciations was 2.25 (range 0-6). In intervention there was a mean decrease of 3.32 mispronunciations per minute from baseline.

Hesitations. During baseline, the mean number of hesitations was 0.43 (range 03). During intervention the mean number of hesitations was 0.35 (range 0-3). In intervention there was a mean decrease of 0.08 hesitations per minute from baseline.

Table 9 (page 89) displays individual mean types of epm in comparison sessions and in generalization probes. The group mean types of epm were as follows:

Omissions. During baseline the mean comparison omissions score for the group was 1.13 (range 0-4). The mean omissions score for baseline generalization was 1.0 (range $0-5$ ). In baseline, the mean generalization score was 0.13 omissions lower than the comparison probe score. 
During intervention the mean comparison omissions score for the group was 0.48 (range 0-3). The mean omissions scores for intervention generalization were 0.31 omissions (range 0-2). In intervention, the mean generalization score was 0.17 omissions lower than the comparison probe score.

During maintenance, the group omissions comparison score was 0.5 (range 0-1). The omissions score for maintenance generalization was 0.75 (range 0-2). In maintenance, the generalization score was 0.25 omissions higher than the comparison probe score.

Additions. During baseline, the comparison additions score for the group was 0.44 (range $0-1$ ). The mean additions score for baseline generalization was 0.56 (range $0-1$ ). In baseline, the mean generalization score was 0.12 additions higher than the comparison probe score.

During intervention, the group mean additions score was 0.21 (range $0-1)$. The mean additions scores for intervention generalization was 0.21 additions (range 0-1). In intervention, the mean generalization score was equal to the comparison probe score.

During maintenance, the group additions mean comparison score was 0.5 (range $0-1$ ). The mean additions score for maintenance generalization was 1.5 (range 0-5). In maintenance, the generalization score was 1 addition higher than the comparison probe score.

Substitutions. During baseline, the comparison mean substitutions score for the group was 2.38 (range 0-7). The mean substitutions score for baseline generalization was 2.94 (range $0-7$ ). In baseline, the mean generalization score was 0.56 substitutions higher than their comparison probe score. 
During intervention, the group mean substitutions score was 2.10 (range 0-6). The mean substitutions scores for intervention generalization was 2.07 (range 0-5). In intervention, the mean generalization score was a mean of 0.3 substitutions higher than the comparison probe score.

During maintenance, the group substitutions comparison score was 2.5 (range 05). The substitutions score for maintenance generalization was 1.25 (range 0-4). In maintenance, the generalization score was 1.25 substitutions lower than the comparison probe score.

Mispronunciations. During baseline the comparison mispronunciations score for the group was 5.44 (range 0-11). The mean mispronunciations score for baseline generalization was 3.13 (range 0-14). In baseline, the mean generalization score was 2.31 mispronunciations lower than the comparison probe score.

During intervention, the group mean mispronunciations score was 3.10 (range 010). The mean mispronunciations score for intervention generalization was 2.69 (range 0 6). In intervention, the mean generalization score was 0.41 mispronunciations lower than the comparison probe score.

During maintenance, the group mean mispronunciation comparison score was 1 (range 0-4). The mispronunciations score for maintenance generalization was 2.5 (range 1-4). In maintenance, the generalization score was 1.5 mispronunciations higher than the comparison probe score.

Hesitations. During baseline, the mean comparison hesitations score for the group was 0.19 (range 0-1). The mean hesitations score for baseline generalization was 0.5 
(range 0-2). In baseline, the mean generalization score was 0.31 hesitations higher than the comparison probe score.

During intervention, the group mean hesitations score was 0.17 (range 0-1). The mean hesitations scores for intervention generalization were 0.48 (range $0-3$ ). In intervention, the mean generalization score was 0.31 hesitations higher than their comparison probe score.

During maintenance, the group mean hesitations comparison score was 0.75 (range 0-2). The mean hesitation score for maintenance generalization was $1(0-3)$. In maintenance, the generalization score was 0.25 hesitations higher than the comparison probe score.

Table 10 (page 90) displays the group's maintenance performance. Maintenance probes were taken 2-, 4-, and 6-weeks after the last intervention session. The group's mean types of epm on their last day of intervention and subsequent maintenance probes were as follows:

Omissions. The last day of intervention mean score was 0.75 (range 0-2) omissions. The group had maintenance mean scores of 0.5 (range 0-1), 0.5 (range 0-1), and 0.75 (range 0-2) omissions respectively. Overall in maintenance, the group committed between 0.25 fewer omissions than the comparison score (weeks 2 and 4 probes) and the same number of omissions as the comparison score (week 6 probe). The group made the fewest omissions the weeks 2 and 4 maintenance probes.

Additions. The last day of intervention mean score was 0.25 (range 0-1) additions. The group had maintenance scores of 0.5 (range $0-1$ ), 0.25 (range $0-1$ ), and 0 additions respectively. Overall in maintenance, the group performed between 0.25 fewer additions 
than the comparison score (week 6 probe) and 0.25 additions more than the comparison score (week 2 probe). The group made the fewest additions in the week 6 maintenance probe.

Substitutions. The last day of intervention mean score was 1.5 (range 0-4) substitutions. The group had maintenance scores of 2.5 (range 0-5), 1.0 (range 0-2), and 2.25 (range 0-5) substitutions respectively. Overall in maintenance, the group performed between 0.5 substitutions fewer than the last session (week 4 probe) and 1 substitution higher than the last session (week 2 probe). The group committed the fewest substitutions on the week 4 maintenance probe.

Mispronunciations. The last day intervention mean score was 2.5 (range 1-4) mispronunciations. The group had maintenance scores of 1.0 (range 0-4), 1.5 (range 0-3), and 3.5 (range 2-5) mispronunciations respectively. Overall in maintenance, the group performed between 1.5 mispronunciations fewer than the last session (week 2 probe) and 1 mispronunciation more than the last session (week 6 probe). The group committed the fewest mispronunciations on the week 2 maintenance probe.

Hesitations. The last day intervention mean score was 0 hesitations. The group had maintenance scores of 0.75 (range 0-2), 1.75 (range 0-5), and 0.25 (range 0-1) hesitations respectively. Overall in maintenance, the group performed between 0.25 more hesitations than the comparison score (week 6 probe) and 1.75 hesitations more than the last session (week 4 probe). The group committed the fewest hesitations on the week 6 maintenance probe. 


\section{Correct Answers to Literal Comprehension Questions}

This study was conducted, in part, to examine the effects of repeated readings on the number of literal comprehension questions answered correctly by ELLs with SLD. The number of correct answers to five literal comprehension questions is presented in the form of individual daily performance and group means.

Generalization scores for correct literal comprehension questions are presented in the form of means and ranges. The results from the generalization probes are then compared to the correct literal comprehension questions means and ranges of the preceding regular sessions. These regular sessions are referred to as the comparison score.

Maintenance scores for correct literal comprehension questions are presented in the form of number of correct literal comprehension questions during each probe. The results from the maintenance probes are then compared to the number of correct literal comprehension questions in the last intervention session.

\section{Tano}

Figure 4 (page 117) displays Tano’s performance in literal comprehension questions during baseline, repeated readings, generalization probes, and maintenance probes. During baseline, Tano's mean number of correct answers to literal comprehension questions was 2.25 (range 1-3). When repeated readings was introduced, Tano's mean number of correct answers to literal comprehension questions increased to 3.63 (range 3-5). Overall, Tano's number of correct answers to literal comprehension questions was highest during the repeated readings intervention, with a mean increase of 1.38 more correct answers over baseline. A summary of individual mean performance on 
number of correct answers to literal comprehension questions can be found in Table 11 (page 118).

Table 12 (page 120) displays individual means for correct literal comprehension questions in comparison sessions and generalization probes. During baseline Tano's comparison score of correct literal comprehension questions was 2 (range 1-3). His mean correct literal comprehension questions score for baseline generalization was 3 (range 33). In baseline, his mean generalization score was 1 correct literal comprehension question higher than his comparison probe score.

During intervention Tano's mean correct literal comprehension questions score was 3.4 (range 2-5). His mean correct literal comprehension questions scores for intervention generalization was 3.6 (range 2-5). In intervention, his mean generalization score was 0.2 correct literal comprehension questions higher than his comparison probe score.

During maintenance, Tano's correct literal comprehension questions comparison score was 3. His correct literal comprehension questions score for maintenance generalization was 2 . In maintenance, his generalization score was 1 correct literal comprehension questions lower than his comparison probe score.

In comparing baseline, intervention and maintenance correct literal comprehension questions generalization scores, Tano had an average of 0.6 more correct literal comprehension questions during intervention generalization probes than baseline generalization probes. Tano's correct literal comprehension questions were highest on his intervention generalization with a mean score of 3.6. 
Tano Baseline $\quad$ Repeated Readings $\quad$ Maint.
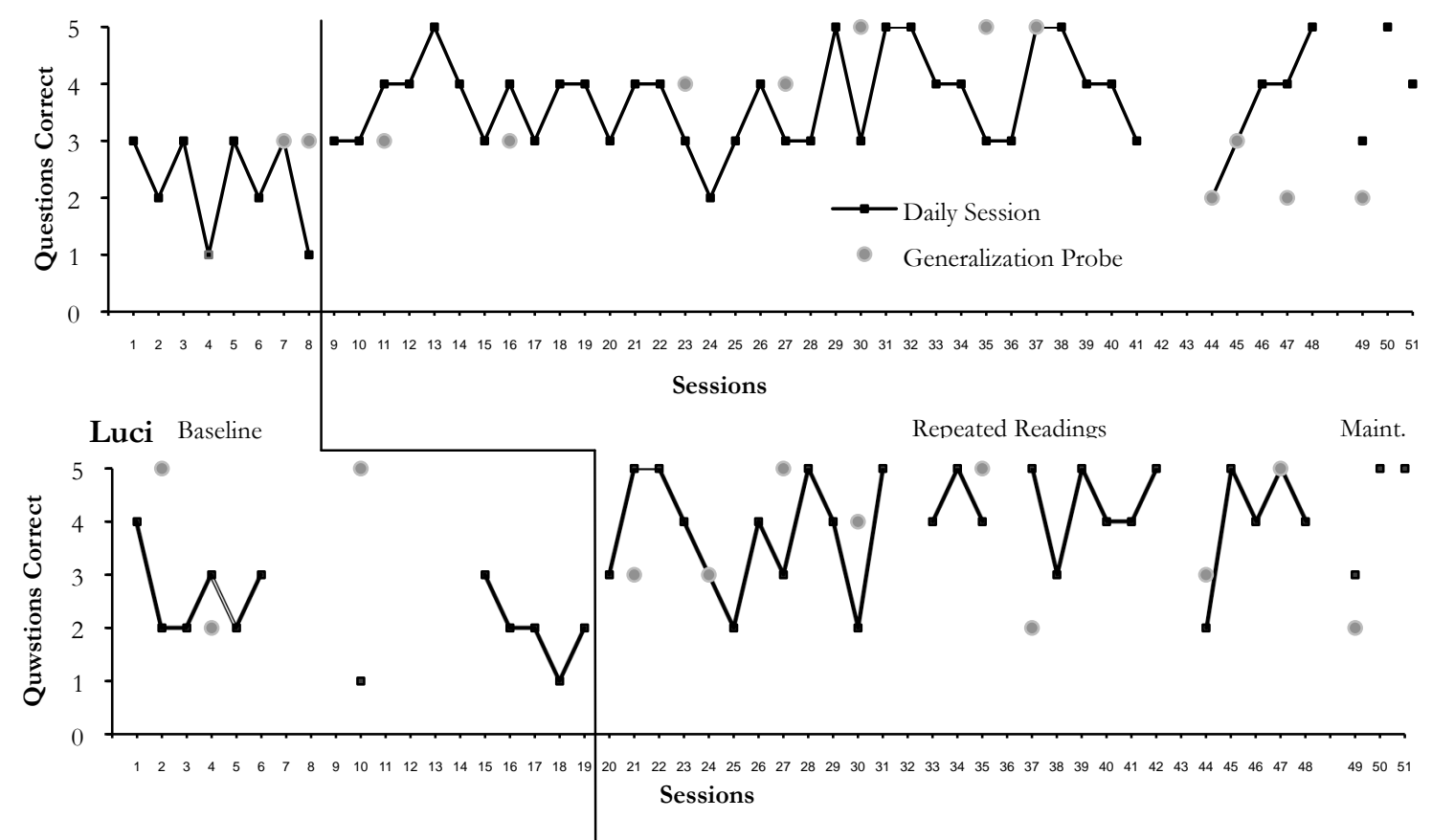

Sandra Baseline

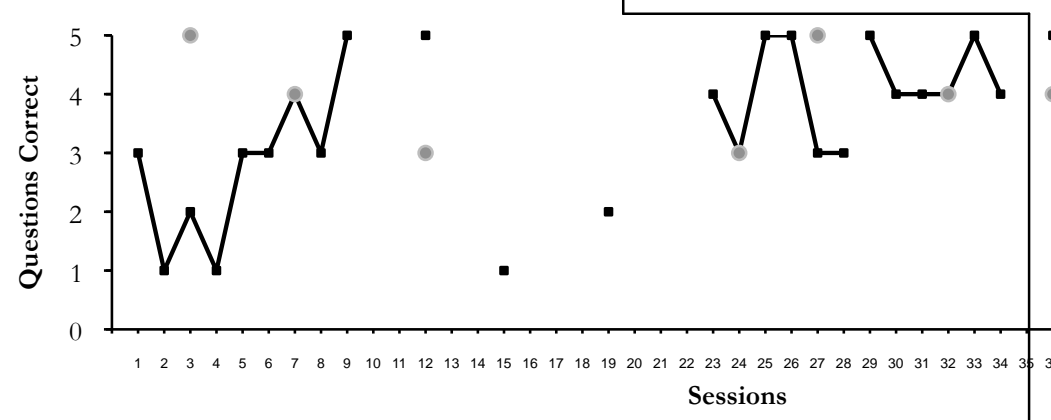

Repeated Readings Maint.

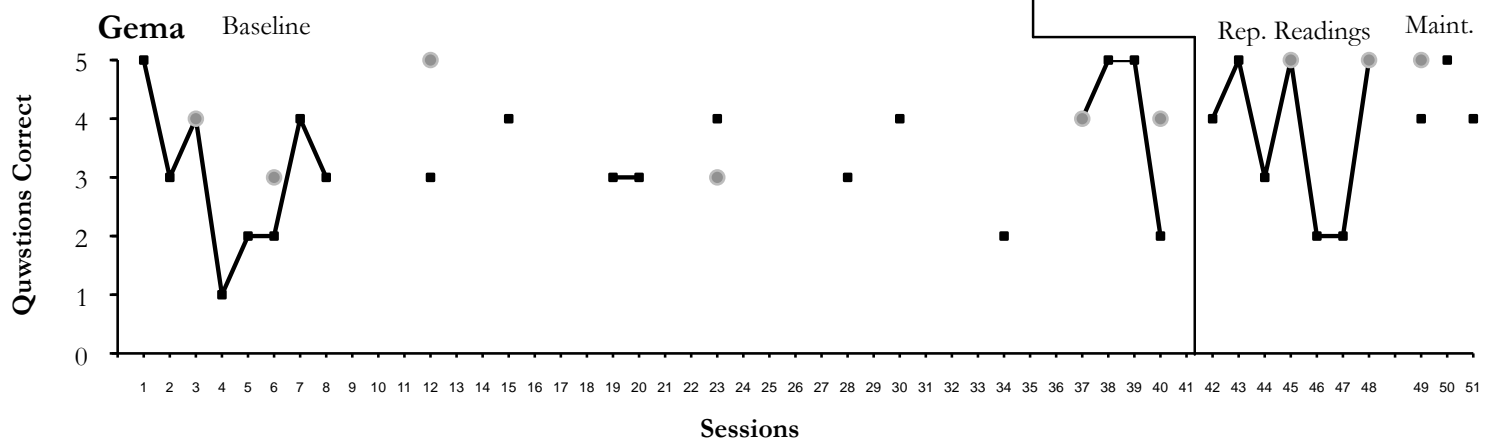

Figure 4. Literal Comprehension as measured by the number of literal comprehension questions answered correctly during the 5-question assessment at the end of each session. Maintenance (Maint.) occurred 2-, 4-, and 6-weeks after the last intervention session. 
Table 11

Individual Means on Correct Answers to Literal Comprehension Questions

\begin{tabular}{ccc}
\hline \multirow{2}{*}{ Participant } & Baseline & Repeated Readings \\
\hline \multirow{2}{*}{ Tano } & 2.25 & 3.63 \\
& $(1-3)$ & $(3-5)$ \\
\multirow{2}{*}{ Luci } & 2.25 & 4.0 \\
& $(1-4)$ & $(2-5)$ \\
Sandra & 3.11 & 4.44 \\
& $(1-5)$ & $(3-5)$ \\
Gema & 3.3 & 3.71 \\
& $(1-5)$ & $(2-5)$ \\
Group & 2.88 & 3.94 \\
Means & $(1-5)$ & $(2-5)$ \\
\hline
\end{tabular}

Note. Top numbers indicate individual mean of correct literal comprehension questions at each phase of the intervention. The bottom rows of numbers represent the range of scores.

Table 13 (page 121) displays Tano’s maintenance performance. Maintenance probes were taken 2-, 4-, and 6-weeks after the last intervention session. Tano's score on his last day of intervention was 3 correct answers to literal comprehension questions. He had maintenance scores of 3, 5, and 4 respectively. Overall in maintenance, Tano maintained between the same score as his comparison score of 3 (week 2 probe) and 2 more questions answered correctly with a score of 5 (week 4 probe). Tano performed highest on his week 4 maintenance probe.

Luci

Figure 4 (page 117) displays Luci’s performance in literal comprehension questions during baseline, repeated readings, and generalization probes. During baseline, Luci's mean number of correct answers to literal comprehension questions was 2.25 
(range1-4). When repeated readings was introduced, Luci's mean number of correct answers to literal comprehension questions increased to 4.0 (range 2-5). Overall, Luci's number of correct answers to literal comprehension questions was highest during the repeated readings intervention, with an increase of 1.75 more correct answers over baseline. A summary of individual mean performance on number of correct answers to literal comprehension questions can be found in Table 11 (page 118).

Table 12 (page 120) displays individual means for correct literal comprehension questions in comparison sessions and generalization probes. During baseline the comparison score of correct literal comprehension questions for Luci was 2 (range 1-3). Her mean correct literal comprehension questions score for baseline generalization was 4 (range 2-5). In baseline, her mean generalization score was 2 correct literal comprehension questions higher than her comparison probe score.

During intervention Luci's mean correct literal comprehension questions score was 3.62 (range 2-5). Her mean correct literal comprehension questions scores for intervention generalization was 3.75 (range 2-5). In intervention, her mean generalization score was 0.13 correct literal comprehension questions higher than her comparison probe score.

During maintenance, Luci's correct literal comprehension questions comparison score was 3. Her correct literal comprehension questions score for maintenance generalization was 2 . In maintenance, her generalization score was 1 correct literal comprehension questions higher than her comparison probe score. 
Table 12

Mean Correct Answers to Literal Comprehension Questions in Comparison Sessions and in Generalization Probes.

\begin{tabular}{|c|c|c|c|c|}
\hline \multicolumn{2}{|c|}{ Participant } & \multicolumn{3}{|c|}{ Mean Correct Literal Comprehension Questions } \\
\hline & & Baseline & Repeated Readings & Maintenance \\
\hline \multirow[t]{2}{*}{ Tano } & $\begin{array}{l}\text { Comparison } \\
\text { Scores }\end{array}$ & $\begin{array}{c}2 \\
(1-3)\end{array}$ & $\begin{array}{c}3.4 \\
(2-5)\end{array}$ & 3 \\
\hline & $\begin{array}{c}\text { Generalization } \\
\text { Probes }\end{array}$ & $\begin{array}{c}3 \\
(3-3)\end{array}$ & $\begin{array}{c}3.6 \\
(2-5)\end{array}$ & 2 \\
\hline \multirow[t]{2}{*}{ Luci } & $\begin{array}{l}\text { Comparison } \\
\text { Scores }\end{array}$ & $\begin{array}{c}2 \\
(1-3)\end{array}$ & $\begin{array}{l}3.62 \\
(2-5)\end{array}$ & 3 \\
\hline & $\begin{array}{c}\text { Generalization } \\
\text { Probes }\end{array}$ & $\begin{array}{c}4 \\
(2-5)\end{array}$ & $\begin{array}{l}3.75 \\
(2-5)\end{array}$ & 2 \\
\hline \multirow[t]{2}{*}{ Sandra } & $\begin{array}{l}\text { Comparison } \\
\text { Scores }\end{array}$ & $\begin{array}{c}3.4 \\
(2-5)\end{array}$ & $\begin{array}{c}4.6 \\
(4-5)\end{array}$ & 5 \\
\hline & $\begin{array}{c}\text { Generalization } \\
\text { Probes }\end{array}$ & $\begin{array}{c}4 \\
(3-5)\end{array}$ & $\begin{array}{c}3.2 \\
(2-5)\end{array}$ & 4 \\
\hline \multirow[t]{2}{*}{ Gema } & $\begin{array}{l}\text { Comparison } \\
\text { Scores }\end{array}$ & $\begin{array}{l}3.17 \\
(2-4)\end{array}$ & $\begin{array}{c}5 \\
(5-5)\end{array}$ & 4 \\
\hline & $\begin{array}{c}\text { Generalization } \\
\text { Probes }\end{array}$ & $\begin{array}{l}3.83 \\
(3-5)\end{array}$ & $\begin{array}{c}5 \\
(5-5)\end{array}$ & 5 \\
\hline \multirow[t]{2}{*}{$\begin{array}{l}\text { Group } \\
\text { Means }\end{array}$} & $\begin{array}{c}\text { Comparison } \\
\text { Scores }\end{array}$ & $\begin{array}{l}3.19 \\
(1-5)\end{array}$ & $\begin{array}{l}3.61 \\
(2-5)\end{array}$ & $\begin{array}{l}3.75 \\
(3-5)\end{array}$ \\
\hline & $\begin{array}{c}\text { Generalization } \\
\text { Probes }\end{array}$ & $\begin{array}{l}3.63 \\
(2-5)\end{array}$ & $\begin{array}{l}3.83 \\
(2-5)\end{array}$ & $\begin{array}{l}3.25 \\
(2-5)\end{array}$ \\
\hline
\end{tabular}

Note. Comparison scores are taken from the regular sessions preceding the generalization probes. Top numbers indicated mean number of Literal Comprehension Questions answered correctly. The bottom rows of numbers represent the ranges of scores.

In comparing baseline, intervention and maintenance correct literal comprehension questions generalization scores, Luci had an average of 0.25 fewer correct literal comprehension questions during intervention generalization probes than 
baseline generalization probes. Luci's correct literal comprehension questions were highest on her baseline generalization probes with a mean score of 4 .

Table 13

Maintenance Performance on Correct Answers to Literal Comprehension Questions

\begin{tabular}{ccccc}
\hline Participant & $\begin{array}{c}\text { Last Intervention } \\
\text { Score }\end{array}$ & $\begin{array}{c}\text { Week 2 } \\
\text { Probe }\end{array}$ & $\begin{array}{c}\text { Week 4 } \\
\text { Probe }\end{array}$ & $\begin{array}{c}\text { Week 6 } \\
\text { Probe }\end{array}$ \\
\hline Tano & 3 & 3 & 5 & 4 \\
Luci & 4 & $(0)$ & $(+2)$ & $(+1)$ \\
& & 3 & 4 & 5 \\
Sandra & 3 & $(-1)$ & $(0)$ & $(+1)$ \\
& & 5 & 5 & 4 \\
Gema & 5 & $(+2)$ & $(+2)$ & $(+1)$ \\
& & 4 & 5 & 4 \\
Group Means & 4 & $(-1)$ & $(0)$ & $(-1)$ \\
& & 3.75 & 4.75 & 4.25 \\
& & $(-0.25)$ & $(+0.75)$ & $(+0.25)$ \\
\hline
\end{tabular}

Note. Top numbers indicate individual number of correct literal comprehension questions on the last day of treatment and on 2-, 4-, and 6-week maintenance probes. The bottom row percentages indicate the percentage of correct literal comprehension questions maintained on 2-, 4-, and 6-week maintenance probes.

Table 13 (page 121) displays Luci's maintenance performance. Maintenance probes were taken 2-, 4-, and 6-weeks after the last intervention session. Luci's score on her last day of intervention was 4 correct answers to literal comprehension questions. She had maintenance scores of 3, 4, and 5 respectively. Overall in maintenance, Luci maintained between 1 question less than her comparison score with a score of 3 (week 2 probe) and 1 more questions answered correctly with a score of 5 (week 6 probe). Luci performed highest on her week 6 maintenance probe. 


\section{Sandra}

Figure 4 (page 117) displays Sandra's performance in literal comprehension questions during baseline, repeated readings, generalization probes and maintenance probes. During baseline, Sandra's mean number of correct answers to literal comprehension questions was 3.11 (range 1-5). When repeated readings was introduced, Sandra's mean number of correct answers to literal comprehension questions increased to 4.44 (range 3-5). Overall, Sandra's number of correct answers to literal comprehension questions was highest during the repeated readings intervention, with an increase of 1.33 more correct answers over baseline. A summary of individual mean performance on number of correct answers to literal comprehension questions can be found in Table 11 (page 118).

Table 12 (page 120) displays individual means for correct literal comprehension questions in comparison sessions and generalization probes. During baseline the comparison score of correct literal comprehension questions for Sandra was 3.4 (range 25). Her mean correct literal comprehension questions score for baseline generalization was 4 (range 3-5). In baseline, her mean generalization score was 0.6 correct literal comprehension questions higher than her comparison probe score.

During intervention Sandra's mean correct literal comprehension questions score was 4.6 (range 4-5). Her mean correct literal comprehension questions scores for intervention generalization was 3.2 (range 2-5). In intervention, her mean generalization score was 1.4 correct literal comprehension questions lower than her comparison probe score. 
During maintenance, Sandra's correct literal comprehension questions comparison score 5. Her correct literal comprehension questions score for maintenance generalization was 4 . In maintenance, her generalization score was 1 correct literal comprehension question lower than her comparison probe score.

In comparing baseline, intervention, and maintenance correct literal comprehension questions generalization scores, Sandra had an average of 0.8 fewer correct literal comprehension questions during intervention generalization probes than baseline generalization probes. Sandra's correct literal comprehension questions were highest on her baseline and maintenance generalization probes with scores of 4 on both.

Table 13 (page 121) displays Sandra's maintenance performance. Maintenance probes were taken 2-, 4-, and 6-weeks after the last intervention session. Sandra's score on her last day of intervention was 3 correct answers to literal comprehension questions. She had maintenance scores of 5, 5, and 4, respectively. Overall in maintenance, Sandra maintained between the 1 more than her comparison score with a score of 4 (week 6 probe) and 2 more questions answered correctly with scores of 5 (weeks 2 and 4 probes). Sandra performed highest on her weeks 2 and 4 maintenance probes.

Gema

Figure 4 (page 117) displays Gema's performance in literal comprehension questions during baseline, repeated readings, generalization probes and maintenance probes. During baseline, Gema's mean number of correct answers to literal comprehension questions was 3.3 (range 1-5). When repeated readings was introduced, Gema's mean number of correct answers to literal comprehension questions increased to 3.71 (range 2-5). Overall, Gema's number of correct answers to literal comprehension 
questions was highest during the repeated readings intervention, with an increase of 0.41 more correct answers over baseline. A summary of individual mean performance on number of correct answers to literal comprehension questions can be found in Table 11 (page 118).

Table 12 (page 120) displays individual means for correct literal comprehension questions in generalization probes. During baseline the comparison score of correct literal comprehension questions for Gema was 3.17 (range 2-4). Her mean correct literal comprehension questions score for baseline generalization was 3.83 (range 3-5). In baseline, her mean generalization score was 0.66 correct literal comprehension questions higher than her comparison probe score.

During intervention Gema's mean correct literal comprehension questions score was 5 (range 5-5). Her mean correct literal comprehension questions scores for intervention generalization was 5 (range 5-5). In intervention, her mean generalization score equal to her comparison probe score.

During maintenance, Gema's correct literal comprehension questions comparison score was 4. Her correct literal comprehension questions score for maintenance generalization was 5. In maintenance, her generalization score was 1 correct literal comprehension question higher than her comparison probe score.

In comparing baseline, intervention and maintenance correct literal comprehension questions generalization scores, Gema had an average of 1.17 more correct literal comprehension questions during intervention generalization probes than baseline generalization probes. Gema's correct literal comprehension questions were 
highest on her intervention and maintenance generalization probes with a score of 5 on both.

Table 13 (page 121) displays Gema's maintenance performance. Maintenance probes were taken 2-, 4-, and 6-weeks after the last intervention session. Gema's last day intervention score was 5 correct answers to literal comprehension questions. She had maintenance scores of 4, 5, and 4 respectively. Overall in maintenance, Gema maintained between one less than her comparison score with a score of 4 (weeks 2 and6 probes) and the same number of questions answered correctly with a score of 5 (week 4 probe). Gema performed highest on her week 4 maintenance probe.

Group Means for Correct Answers to Literal Comprehension Questions

Table 11 (page 118) displays group performance means in literal comprehension questions during baseline and repeated readings. During baseline, the mean number of correct answers to literal comprehension questions was 2.88 (range 1-5). When repeated readings was introduced, the mean number of correct answers to literal comprehension questions increased to 3.94 (range 2-5). Overall, the mean number of correct answers to literal comprehension questions was highest during the repeated readings intervention, with a mean increase of 1.21 more correct answers over baseline.

Table 12 (page 120) displays group means for correct literal comprehension questions in comparison sessions and generalization probes. During baseline the comparison score of correct literal comprehension questions for the group was 3.19 (range 1-5). The mean correct literal comprehension questions score for baseline generalization was 3.63 (range 2-5). In baseline, the mean generalization score was 0.44 correct literal comprehension questions higher than their comparison probe score. 
During intervention the group mean correct literal comprehension questions score was 3.61 (range 2-5). The mean correct literal comprehension questions scores for intervention generalization was 3.83 (range 2-5). In intervention, their mean generalization score was 0.22 correct literal comprehension questions higher than the comparison probe score.

During maintenance, the group mean correct literal comprehension questions comparison score was 3.75 (range 3-5). The correct literal comprehension questions score for maintenance generalization was 3.25 (range 2-5). In maintenance, the generalization score was 0.50 correct literal comprehension questions lower than their comparison probe score.

In comparing baseline, intervention and maintenance correct literal comprehension questions generalization scores, the group had an average of 0.2 more correct literal comprehension questions during intervention generalization probes than baseline generalization probes. The group's correct literal comprehension questions were highest on their intervention generalization with a mean score of 3.83 (range 2-5).

Table 13 (page 121) displays the group's maintenance performance. Maintenance probes were taken 2-, 4-, and 6-weeks after the last intervention session. The group's last day mean intervention score was 4 correct answers to literal comprehension questions. The group had mean maintenance scores of 3.75, 4.75, and 4.25 respectively. Overall in maintenance, the group maintained between 0.25 fewer correct answers than their comparison score of 4 (week 2 probe) and 0.75 more questions answered correctly with a score of 4.75 (week 4 probe). The group performed highest on their week 4 maintenance probes. 


\section{Summary}

The results of this study indicate that the participants improved their reading fluency, decreased the number of errors committed aloud, committed fewer of the five types of errors, and improved their ability to answer literal comprehension questions when using repeated readings to read passages of approximately 100 words as compared to baseline.

Generalization data were mixed for the different dependent variables, however, generalization means for intervention and maintenance consistently remained higher than baseline generalization means. For fluency, generalization wpm scores were higher than comparison probe scores in baseline and maintenance. For epm, generalization scores indicated slightly more epm in generalization than comparison scores across baseline, intervention, and maintenance conditions. For types of epm, more additions and hesitations were committed during generalization probes thank comparison probes in baseline, intervention, and maintenance. Additionally, there were more omissions and mispronunciation errors in generalization probes following maintenance sessions. There were fewer omissions, substitutions, and mispronunciations in generalization probes as compared to comparison scores following baseline and intervention sessions. For correct answers to literal comprehension questions, generalization probes were slightly higher in baseline and intervention than comparison scores. However, in maintenance, the mean generalization probe was slightly lower than the comparison score taken on the last day of intervention.

Maintenance data for the different dependent variables demonstrated that the maintenance means remained above baseline mean levels. When compared to baseline, 
participants read more words per minute in maintenance probes. Additionally, participants committed fewer errors in maintenance probes than in baseline. Participants, on average, committed fewer omissions, fewer additions, fewer substitutions, and fewer mispronunciations in maintenance probes than in baseline. However, they committed more hesitations in maintenance than in baseline. Participants on average answered more literal comprehension questions correctly in maintenance than in baseline. 


\section{CHAPTER V \\ DISCUSSION}

This chapter provides a discussion on the results of this study. A brief study overview is followed by a summary of the results with respect to relevant literature in repeated readings. The study's delimitations, limitations, implications for practice, and suggestions for future research are also included.

This study examined the effects of repeated readings on the reading skills of four, third-, fourth-, and fifth-grade English language learners (ELLs) with specific learning disabilities (SLD). Data were collected and analyzed on a total of 58 baseline probes, 89 intervention sessions, 45 generalization sessions, and 12 maintenance reading sessions across 4 participants and four dependent variables: reading fluency (words read aloud correctly per minute; wpm), number of errors read aloud per minute (epm), types of errors read aloud per minute, and the number of correctly answered literal comprehension questions. Generalization probes were taken following $28.3 \%$ of all sessions.

Maintenance probes were taken 2-, 4-, and 6-weeks after the end of the study.

Although outcomes varied for individual participants, overall the results demonstrated that repeated readings had a positive impact on participant reading skills as measured by the dependent variables. Participant generalization scores were higher in intervention than in baseline. Additionally, with the exception of the number of hesitations committed per minute of reading, maintenance probes on the dependent variables indicated that participant scores remained above baseline levels. A detailed 
analysis of the results for each dependent variable, along with comparisons to previous research follows.

\section{Reading Fluency}

With variation, all four participants read more words per minute during the repeated readings intervention than during baseline. Tano, Luci, Sandra, and Gema read a mean of $17.21,30.48,28.08$, and 14.95 more wpm respectively in intervention than in baseline.

These results support the work of Nelson et al. (2004), whose study demonstrated that repeated readings assisted students with disabilities to read more fluently. In the Nelson et al. study, participants read a mean of 19.67 more wpm when repeated readings was introduced in an error correction treatment. In this study, participants read more wpm when repeated readings were introduced, with a mean of 22.68 more wpm. Similarly, Tam et al., (2006) found that repeated readings had a positive effect on reading fluency in ELLs reading below grade level, with participants reading a mean of up to 37.8 more wpm than baseline when they repeatedly read the same passage up to a previously determined criterion.

When comparing generalization probes to comparison probes (the data point taken the same days as the generalization probes), participant generalization scores tended to be slightly higher than comparison scores in baseline and slightly lower than comparison scores in intervention. However, with the exception of one data point, participant generalization scores were higher in intervention than in baseline.

Maintenance scores showed high variability among all four participants. All four, however, maintained more than $57 \%$ of their gains in intervention. When compared to 
baseline, participants read a mean of $62.63 \%$ more wpm in maintenance sessions. Tano and Luci had the highest maintenance scores. Tano and Gema, in their weeks 4 and 6 probes respectively both read slightly more wpm than in their last intervention sessions. This variation may be due to an interest in the passage topic that day (Clark, 1995). It may also be indicative of additional reading practice in the weeks following intervention.

In summary, the results suggest that for ELLs with SLD, repeated readings can result in an increase reading fluency. While the method led to higher wpm in intervention and generalization as compared to baseline, the percentage of generalization dropped when the passages were read repeatedly. The results of this study also suggest that repeated readings leads to some maintenance in reading fluency gains once the method is not employed. Tam et al. (2006), also found that the higher level of fluency gained in repeated readings interventions may have led to maintenance in fluency gains. In contrast, Nelson et al. (2004) found that for three out of four participants, maintenance scores were only slightly higher than baseline wpm scores.

\section{Errors Per Minute}

Participants committed fewer reading errors during the repeated readings intervention than during baseline. The impact of the intervention, however, varied. All four participants made fewer errors per minute when the repeated readings was employed. Tano, Luci, Sandra, and Gema made a mean of 3.16, 4.04, 3.50, and 3.66 fewer errors per minute respectively in intervention than in baseline.

Generalization probes indicated that the number of participant errors remained similar in comparison and generalization probes throughout the study. This indicates that there was generalization from baseline to intervention in epm. 
Maintenance scores showed variability among all four participants. Two participants committed the same or fewer errors than on their last intervention session in all three maintenance sessions. There was no other discernable pattern in the number of errors committed on the last day of intervention in comparison with the number of errors committed in maintenance probes. However, when compared to baseline, participants read a mean of $61.56 \%$ fewer epm in maintenance. This is in contrast to Nelson et al., (2004), who found that epm were only slightly lower than baseline for all four participants when previously read materials were used in the final condition of the study.

Given that overall there was a decrease in errors per minute with repeated readings, the data suggest that this method has a positive effect on the number of errors committed by ELLs with SLD. These results support the work of Nelson et al. (2004), whose study demonstrated that repeated readings helped students with disabilities read with fewer errors. Additionally, one previous study found that repeated readings had a positive effect on errors per minute in ELLs reading below grade level (Tam et al., 2006). The results of the study by Tam et al. (2006) and the current study found that the number of epm decreased from baseline when repeated readings was implemented.

In summary, the results suggest that for ELLs with SLD, repeated readings should be considered a strategy to decrease reading errors. The results of this study suggest that when compared to baseline, repeated readings leads to maintenance in error reduction. While repeated readings leads to lower error rates in intervention and generalization, generalization in the area of epm needs to be further examined. Subsequently, the results of this study should be viewed as preliminary. 


\section{Types of Errors Per Minute}

Participants committed fewer reading errors during the repeated readings intervention, however, the impact of the intervention on the types of errors committed per minute varied. In this study, five types of errors were identified: omissions, additions, substitutions, mispronunciations, and hesitations. The most affected reduction in errors across all four participants from baseline to intervention occurred in the category of mispronunciations. Tano, Luci, Sandra, and Gema decreased their number of mispronunciations per minute by $2.71,4.66,2.41$, and 3.5 respectively from baseline to intervention. One participant (Tano) committed fewer of each type of error in intervention as compared to baseline.

In comparing generalization probes to comparison scores there is a great deal of variance (see Table 9, page 89). In the category of omissions, all four participants committed the same number of omissions or fewer in intervention generalization probes than comparison scores. In contrast, in the category of hesitations, all four participants committed more hesitations in intervention generalization probes than in comparison scores.

Maintenance scores showed high variability among all four participants. Only two participants (Tano and Luci) demonstrated any consistency in maintenance of the reduction in the types of errors committed per minute. Tano committed no hesitations in his last session and remained consistent in all of his maintenance probes. Luci committed no additions or hesitations in her last session and remained consistent in all of her maintenance probes. However, on average maintenance scores indicated a reduction in omissions, additions, substitutions, and mispronunciation when compared to baseline. 
Taken cautiously, the results of this study indicate that for these four participants, repeated readings led to a decrease in the number of mispronunciation errors committed per minute. These results were not evident in generalization probes. While many studies that have implemented a repeated readings intervention have clearly defined what constituted an error, these studies did not record the number of each type of error committed by participants (e.g., Alber-Morgan et al., 2007; Tam et al., 2006). No other study was found that examined the effects of repeated readings on the types of errors committed per minute. Subsequently, the results of this study should be viewed as preliminary.

\section{Correct Answers to Literal Comprehension Questions}

Repeated readings led to an improvement in the number of literal comprehension questions answered correctly for all four participants. Out of a total of five questions asked, Tano, Luci, Sandra, and Gema answered a mean of 1.38, 1.78, 1.33, and 0.41 more literal comprehension questions respectively during intervention than in baseline.

Results from generalization probes were varied. Only two participants had higher generalization scores than comparison scores in baseline and in intervention. The variation in generalization scores, as well as the fact that one participant answered all of her questions correctly following several sessions may mean that more literal comprehension questions are necessary in order to be able to draw further conclusions about the effects of repeated reading on the number of correct literal comprehension questions answered correctly.

Maintenance scores showed variability among all four participants. In maintenance, two participants had maintenance scores that were the same or higher than 
the comparison scores taken on the last day of intervention. The other two participants, had maintenance scores that were the same or lower than their comparison scores. Maintenance means were on average $47.06 \%$ above baseline means.

Given that overall performance improved in literal comprehension questions, the data suggest that repeated readings has a positive effect on the ability of ELLs with SLD to answer literal comprehension questions correctly. Similarly, Alber-Morgan et al., (2007) witnessed an immediate increase in the number of literal comprehension questions answered correctly by three out of four students with behavior problems after implementing a repeated readings intervention. Tam et al. (2006) asked four participants literal comprehension questions during generalization probes and found that an increased reading rate in participants following a repeated readings intervention may lead to an increase in comprehension in taught and untaught passages.

In summary, the results suggest that for ELLs with SLD, repeated readings may increase reading comprehension as measured by literal comprehension questions. However, few studies have examined the effects of a repeated readings intervention on the participants' ability to answer literal comprehension questions, therefore these results should be interpreted cautiously and viewed as preliminary.

\section{Repeated Readings Summary}

Overall, the results of this study lend further support to the limited research which demonstrated that repeated readings has a positive impact on the reading abilities of ELLs with SLD (Tam et al., 2006). In this study, when repeated readings was implemented, participants read more words per minute, made fewer errors per minute, and answered more literal comprehension questions correctly. Additionally, participants 
committed fewer mispronunciation errors with repeated readings as compared to baseline. Inconsequential results on the number of omissions, additions, substitutions, and hesitations from baseline to intervention were observed. Generalization probes in intervention were higher than generalization probes in baseline. Maintenance scores were variable, however they were consistently stronger than baseline means.

The results of this study support the work of Tam et al., (2006) who suggests that a structured reading intervention incorporating repeated readings has a positive effect on reading fluency and comprehension on ELLs who are struggling readers. As no previous research was found that specifically targeted ELLs with SLD, subsequently, no connections to previous research can be made. The results of this study, however, preliminarily suggest that repeated readings has a positive effect on the reading abilities of ELLs with SLD.

This study extends the literature on the effects of repeated readings on ELLs with SLD. Prior to this study, only one study was found that included participants who were ELLs with SLD (Tam et al., 2006). Additionally, this limited research was conducted with only two of the four participants being labeled as ELLs with SLD struggling with reaing and one of those two was actually found to be reading at grade level during the course of the study. This study added to the literature by providing an analysis of the types of errors participants made in every phase of the study. Previous research had defined what constituted an error but had not reported on the different types of errors participants were making. This study extends the literature in that it was conducted in a large urban school district with Hispanic students whereas previous studies were conducted in a rural school district with students who were not identified as ELLs 
(Nelson et al., 2004) and with ELLs from varying ethnic backgrounds (Tam et al., 2007). This study also extended the literature in that it examined the effects of repeated readings on participants' ability to answer literal comprehension questions correctly. Many previous research studies only explored the effects of repeated readings on fluency and few have examined its effects on comprehension (Alber-Morgan et al., 2007; Tam et al., 2006).

\section{Implications for Practice}

The results of this study have important implications for classroom practice. The methods used in this study are effective in improving reading and can be a valuable supplemental reading instruction for teachers to employ with their students who are ELLs with SLD. Additionally, it is cost efficient and easy to implement. Teachers may implement this method one on one or with a small group of students, or they may train paraprofessionals, volunteers, or competent students to do it (Alber-Morgan et al., 2007). Parents can also be taught to repeatedly read short passages with their children at home.

The results of this study may also have implications for how instructional reading time is used. This study used 10-20 minute reading sessions in which gains in fluency were noted as soon as repeated readings were introduced. Teachers may want to assign shorter passages to ELLs with SLD to allow for repeated readings during class time. Additionally, teachers may encourage students to repeatedly read a passage before attempting reading assessments and may therefore extend time for those assessments if possible.

Previous research has shown that ELLs and students with SLD benefit from interventions that include vocabulary instruction (Fitzgerald, 1995; Gersten \& Baker, 
2000; Tam et al., 2006), repeated readings (Chard et al., 2002; Nelson et al., 2004;

Therrien \& Kubina, 2006; Weinstein \& Cooke, 1992), and individual attention (Elbaum et al., 2000). The current study used an intervention method that encompassed those elements resulting in improved reading fluency, reduced reading errors, and an improvement in the overall number of comprehension questions answered correctly in four ELLs with SLD. These results further stress the need for structured research based interventions to be used with students who are struggling with reading in general, and ELLs with SLD in particular.

Only one previous study collected generalization and maintenance data in conjunction with a repeated readings intervention. Tam et al. (2006) collected comprehension data during generality probes, however, the probes did not require the participants to read aloud, therefore, fluency was not measured. This study added to those findings by collecting generalization data on all of the dependent variable measured in the study. Tam et al. also collected maintenance data following the conclusion of their study. Similar to the present study, Tam et al. found that the fluency reached in intervention might lead to greater fluency once the intervention is withdrawn. The present study's findings further stress the need to examine the effects that repeated readings have on the maintenance and generalization of reading gains in ELLs with SLD.

\section{Delimitations}

The sample size used in single subject design research is small by the nature of the design and therefore limits the generalization of its findings. In order to determine the effects of repeated readings on the reading skills of ELLs with SLD direct and systematic replications are needed. For example, this study included only third-, fourth-, and fifth- 
grade, Hispanic ELLs labeled as SLD. Therefore the results cannot be generalized to nonHispanic participants at other grade levels, or with other abilities or disabilities.

The present study was delimited to passages that were previously prepared from a basal reading series. If the student did not connect to the reading passage, it is possible that his or her participation was negatively impacted. While they were of the same readability level, the passages had a variety of topics and were written by various authors. This being said, no participant expressed a preference or distaste of any passage.

An additional delimitation was that participants in this study were participating during the course of a normal school day. Participants were pulled from their classrooms during a period in their schedule when they were participating in silent reading with the exception of one student who was participating during homeroom. Although each student had his or her session at the same time every day, sessions sometimes occurred at moments when the students either wanted to or did not want to leave the classroom. Though notes were recorded whenever a student verbalized feelings of wanting to go or not go to the session, data were not collected on this. This may have had effects on the participants' performances.

\section{Limitations}

Several study limitations should be noted. Though participants met with the researcher prior to the start of the study, both the researcher and the scenario of receiving additional reading practice during the day were novel to all four participants. Given this, it may have taken some time for the participants to get comfortable with the researcher. Also, the additional adult attention allotted by the intervention may have impacted the results. Time constraints (along with a review of the data) needed to be taken into 
consideration in determining when to implement some of the phases. Specifically, Gema only had 7 days of intervention sessions. Had time not been an issue, Gema's intervention phase may have been extended until more stability was observed. While Gema's limited exposure to the intervention may be a limitation to the study, her prolonged baseline phase actually demonstrates the strength of the intervention. Gema's performance in baseline remained steady, even as she was receiving reading instruction in the classroom. She made gains in fluency once the intervention was introduced just as the other participants who had a shorter baseline phase.

Another limitation of this study is that previous and daily reading instruction may have impacted participants' performance. The participants in this study received daily reading instruction in three separate settings from six different teachers. Though the teachers followed a designated curriculum, the content and quality of the teaching was not observed. Control and/or an analysis of the reading instruction participants received during the study may have impacted the effects of the interventions, however relatively stable baseline data gave the researcher confidence in the intervention's effectiveness. It would have been useful to have classroom daily reading instruction data to further interpret the results.

\section{Suggestions for Future Research}

The results of this study suggest several areas for future research. The demographic characteristics of the participants that took part in this study were restricted to third-, fourth-, and fifth-grade, Hispanic ELLs who were labeled as having an SLD. Participants with other demographic characteristics should be considered in future research such as non-Hispanic ELLs, native English speakers, students differing in 
abilities and disabilities, and students at different grade levels. Additionally, future research can examine how many repeated readings are optimal for ELLs with SLD and whether reading to a set number of words per minute leads to higher reading gains.

Future research should monitor the reading instruction taking place in the classroom during the study to determine if it impacts the effects of repeated readings. Further investigation should also investigate the effects of repeated readings alone and/or in combination with other research based practices such as having students read with a competent peer tutor (Chard et al., 2002), or having students track their own progress (Tam et al., 2007). Additionally, different forms of error correction can be employed with repeated readings such as phrase drill error correction (Begeny et al., 2006) or systematic error correction (Nelson et al., 2004).

Although outcomes have varied for individual participants, data demonstrate that repeated readings have had a positive impact on participant reading skills as measured by the dependent variables. The results suggest that for ELLs with SLD, repeated readings increased reading fluency, decreased the number of errors, and increased the number of correct literal comprehension questions related to the reading.

This study examined the use of repeated readings and students who were ELLs with SLD; however, there is a need for additional research. No known previous study has specifically targeted ELLs with SLD who are struggling readers using the methods in this study. The sample size is small by the nature of the design and therefore limits the generalization of its findings. Additional research may focus on ELLs with SLD from non-Hispanic backgrounds. Although this study targeted students reading at least 1 year below grade level, future studies could examine ELLs with SLD at various stages of 
reading acquisition and various stages of their English language acquisition. Additionally, more research is needed in the area of repeated readings and the number of readings that is optimal for ELLs with SLD.

\section{Summary}

The results of this study suggest that repeated readings has a positive impact on the reading abilities of ELLs with SLD. Reading fluency, number of errors read aloud per minute, and literal comprehension question results demonstrated that participants read more words, made fewer errors, and obtained higher literal comprehension question scores when repeated readings was employed. Additionally, participants made fewer mispronunciation errors once repeated readings were introduced.

Participants read passages in their instructional reading level one time during baseline and three times repeatedly in intervention. The sessions were recorded and recordings were analyzed across four dependent variables: reading fluency (words read aloud correctly per minute), number of errors read aloud per minute, types of errors read aloud per minute, and the number of correctly answered literal comprehension questions. Generalization probes were taken and compared to comparison scores. Maintenance probes were taken 2-, 4-, and 6-weeks after the end of the study.

Reading fluency results showed that participants in read more words per minute when repeated readings were introduced. Additionally, generalization wpm scores in intervention and maintenance wpm scores remained higher than baseline wpm scores.

Results in epm indicate that participants made fewer errors when repeated readings was introduced. The generalization probes indicated that error rates did not 
return to baseline levels on similar untaught passages. Maintenance data in epm was varied but epm means remained lower than mean baseline levels.

Results in types of epm indicate that participants made fewer mispronunciation errors when repeated readings was introduced. The generalization probes in types of epm was varied; no discernable pattern could be detected. In maintenance, participants committed on average fewer omission errors, addition errors, substitution errors, and mispronunciation errors than in baseline.

The results in correct answers to literal comprehension questions showed that participants answered more literal comprehension questions correctly when repeated readings were introduced. Generalization data were varied, and may have been affected by the fact that only five questions were asked per passage, creating a ceiling effect. Maintenance scores were varied, however participant scores remained above baseline means.

The overall results of this study suggest that repeated readings could assist ELLs with SLD who are struggling with reading in English with improving their reading skills. This study adds to the limited research on ELLs with SLD by demonstrating the effects of repeated readings on this population, as well as, by conducting an analysis of the types of errors these students committed when reading aloud. Additionally, it examined generalization and maintenance data, which has not been measured in most repeated readings research. This study lends further support for the use of a structured supplemental reading instruction program employing repeated readings with ELLs with SLD who are struggling with reading in English. 
Since this study explored the effects of repeated readings on third-, fourth-, and fifth-grade Hispanic ELLs in ESOL levels III, IV, and V, with SLD, results cannot be generalized beyond this population. Future research should explore its efficacy with diverse participant populations. Furthermore, future research can investigate the effects of repeated readings on ELLs with SLD at different stages of reading acquisition. This study determined that repeated readings can have positive effects on the reading skills of ELLs with SLD and therefore should be considered as a tool to assist these students in improving their reading abilities. 


\section{REFERENCES}

Abidin, R. R., \& Robinson, L. L. (2002). Stress, biases, or professionalism: What drives teachers' referral judgments of students with challenging behaviors? Journal of Emotional and Behavioral Disorders, 10, 204-212.

Alber-Morgan, S. R., Ramp, E. M., Anderson, L. L., \& Martin, C. M. (2007). Effects of repeated readings, error correction, and performance feedback on the fluency and comprehension of middle school students with behavior problems. The Journal of Special Education, 41, 17-30.

Albus, D., Thurlow, M., \& Clapper, A. (2007). Standards-based instructional strategies for English language learners with disabilities. (ERIC Document Reproduction Service No. ED499409)

American College Testing Program. (2006). Ready for college and ready for work: Same or different? Retrieved February 1, 2008, from http://www.act.org/research/ policymakers/reports/workready.html

Barbetta, P. M., Heward, W. L., \& Bradley, D.M. (1993). Relative effects of whole-word and phonetic-prompt error correction on the acquisition and maintenance of sight words by students with developmental disabilities. Journal of Applied Behavior Analysis, 26, 99-110.

Barley, Z., Lauer, P. A., Arens, S. A., Apthorp, H. A., Englert, K. S., Snow, D., et al. (2002). Helping at-risk students meet standards: A synthesis of evidence-based classroom practices. Aurora, CO: Mid-Continent Research for Education and Learning.

Barton, K. E., \& Huynh, H. (2003). Patterns of errors made by students with disabilities on a reading test with oral reading administration. Educational and Psychological Measurement, 63, 602-614.

Begeny, J. C., Daly, E. J., III, \& Valleley, R. J. (2006). Improving oral reading fluency through response opportunities: A comparison of phrase drill error correction with repeated readings. Journal of Behavioral Education, 15(4), 229-235.

Bernhard, J. K., Cummins, J., Campoy, F. I., Ada, A. F., Winsler, A., \& Bleiker, C. (2006). Identity texts and literacy development among preschool English language learners: Enhancing learning opportunities for children at risk for learning disabilities. Teachers College Record, 108, 2380-2405. 
Boulineau, T., Fore, C., Hagan-Burke, S., \& Burke, M.D. (2004). Use of story-mapping to increase the story-grammar text comprehension of elementary students with learning disabilities. Learning Disability Quarterly, 27, 105.

Brisk, M.E. \& Harrington, M.M. (2000). Literacy and bilingualism: A handbook for all teachers. Mahwah, NJ: Lawrence Elbaum Associates Publishing.

Brown, B. V., \& Bogard, K. (2007). Pre-kindergarten to 3rd grade (PK-3) school-based resources and third grade outcomes. Retrieved February 1, 2008, from http://www.childtrendsdatabank.org/PDF/PKtoThree.pdf

Bryant, D.P., Vaughn, S., Linan-Thompson, S., Ugel, N., Hamff, A., \& Hougen, M. (2000). Reading outcomes for students with and without reading disabilities in general education middle-school content area classes. Learning Disability Quarterly, 23, 238-252.

Case, R. E., \& Taylor, S.S. (2005). Language difference or learning disability? Answers from a linguistic perspective. The Clearing House, 78, 127.

Chard, D. J., Vaughn, S., \& Tyler, B. (2002). A synthesis of research on effective interventions for building reading fluency with elementary students with learning disabilities. Journal of Learning Disabilities, 35, 386-406.

Clark, C.H. (1995). Teaching students about reading: A fluency example. Reading Horizons 35, 250-266.

Cooper, J., Heron, T., \& Heward, W. (2007). Applied behavior analysis ( $2^{\text {nd }}$ ed.). Upper Saddle River, NJ: Pearson/Merrill-Prentice Hall.

Dade County Board of Public Instruction. (1978). Oral language proficiency scale. (ERIC Document Reproduction Service No. ED179566)

Denton, C. A., Anthony, J. L., Parker, R., \& Hasbrouck, J.E. (2004). Effects of two tutoring programs on the English reading development of Spanish-English bilingual students. The Elementary School Journal, 104, 289.

Dictionary.com. (2008). Retrieved March 14, 2008, from http://dictionary.reference.com/ search? $\mathrm{r}=2 \& \mathrm{q}=$ Mispronunciation

Elbaum, B., Vaughn, S., Hughes, M. T., \& Moody, S. W. (2000). How effective are oneto-one tutoring programs in reading for elementary students at risk for reading failure? A meta-analysis of the intervention research. Journal of Educational Psychology, 92, 605-619. 
Florida Department of Education. (2006-2007). Florida School Indicators Report. Retrieved February 1, 2008, from http://data.fldoe.org/fsir/default.cfm

Florida Department of Education. (2008). Florida comprehensive assessment test: Student performance results demographic report, 2000-2007. Retrieved February 1, 2008, from http://www.fcatresults.com/demog/

Fitzgerald, J. (1995). English-as-a-second-language learners' cognitive reading processes: A review of research in the United States. Review of Educational Research, 65, $145-190$.

Freeland, J. T., Skinner, C. H., Jackson, B., McDaniel, C. E., \& Smith, S. (2000). Measuring and increasing silent reading comprehension rates: Empirically validating a repeated readings intervention. Psychology in the Schools, 37, 415429 .

Fry, E., Fountoukidis, D., \& Polk, J. (1985). The new reading teacher's book of lists ( $2^{\text {nd }}$ ed.). Englewood Cliffs, NJ: Prentice-Hall.

Fry, R. (2007). How far behind in math and reading are English language learners. Retrieved February 1, 2008, from http://pewhispanic.org/reports/ report.php?ReportID=76

Gersten, R., \& Baker, S. (2000). What we know about effective instructional practices for English-language learners. Exceptional Children, 66, 454-470.

Gersten, R., Fuchs, L. S., Williams, J.P., \& Baker, S. (2001). Teaching reading comprehension strategies to students with learning disabilities: A review of research. Review of Educational Research, 71, 279-320.

Gersten, R., \& Woodward, J. (1994). The language-minority student and special education: Issues, trends, and paradoxes. Exceptional Children, 60, 310-322.

Good, R. H., \& Kaminski, R. A. (Eds.). (2002). Dynamic indicators of basic early literacy skills: DIBELS (6th ed.). Eugene, OR: Institute for Development of Educational Achievement.

Hitchcock, C. H., Prater, M. A., \& Dowrick, P. W. (2004). Reading comprehension and fluency: Examining the effects of tutoring and video self-modeling on first-grade students with reading difficulties. Learning Disability Quarterly, 27, 89-103.

Krashen, S. D., \& Terrell, T. D. (1983). The natural approach: Language acquisition in the classroom. (ERIC Document Reproduction Service No. ED230069) 
LaBerge, D. \& Samuels, S. J. (1974). Toward a theory of automatic information processing in reading. Cognitive Psychology, 6, 293-323.

Lazarus, D., \& Callahan, T. (2000). Attitudes toward reading expressed by elementary school students diagnosed with learning disabilities. Reading Psychology, 21, 271-282.

Linan-Thompson, S., Vaughn, S., Hickman-Davis, P., \& Kouzekanani, K. (2003). Effectiveness of supplemental reading instruction for second-grade English language learners with reading difficulties. Elementary School Journal, 103, 221238.

Manset-Williamson, G., \& Nelson, J. M. (2005). Balanced, strategic reading instruction for upper-elementary and middle school students with reading disabilities: A comparative study of two approaches. Learning Disability Quarterly, 28, 59-74.

McCardle, P., Mele-McCarthy, J., \& Leos, K. (2005). English language learners and learning disabilities: Research agenda and implications for practice. Learning Disabilities Research \& Practice, 20, 68-78.

Miami-Dade County Public Schools. (2005). Guide to completing an individual LEP student plan: Elementary. Retrieved March 14, 2008, from http://bilingual.dadeschools.net/BEWL/pdf/Individual_LEP_Plan.pdf

Miami-Dade County Public Schools (2006). 2006-07 District and School Profiles. Retrieved March 14, 2008, from http://oada.dadeschools.net/DSProfiles/ 0607Profiles.asp

Miami-Dade County Public Schools (2007). Policies and procedures for the provision of specially designed instruction and related services for exceptional students. Retrieved March 14, 2008, from http://ehandbooks.dadeschools.net/policies/ 149.pdf

Nathan, R. G., \& Stanovich, K. E. (1991). The causes and consequences of differences in reading fluency. Theory into Practice, 30, 176-184.

Nelson, J. S., Alber, S. R., \& Gordy, A. (2004). Effects of systematic error correction and repeated readings on the reading accuracy and proficiency of second-graders with disabilities. Education \& Treatment of Children, 27, 186-198.

Osborn, J., Freeman, A., Burley, M., Wilson, R., Jones, E., \& Rychener, S. (2007). Effect of tutoring on reading achievement for students with cognitive disabilities, specific learning disabilities, and students receiving Title I services. Education \& Training in Developmental Disabilities, 42, 467-474. 
Pattillo, S. T., Heller, K. W., \& Smith, M. (2004). The impact of a modified repeatedreading strategy paired with optical character recognition on the reading rates of students with visual impairments. Journal of Visual Impairment and Blindness, 98, 28-46.

Pressley, M., Gaskins, I., \& Fingeret, L. (2006). Instruction and development of reading fluency in struggling readers. In S.J. Samuels \& A.E. Farstrup (Eds.), What research has to say about fluency instruction (pp.47-69). Newark, DE: International Reading Association.

Pugh, K. R., Sandak, R., Frost, S. J., Moore, D., \& Mencl, W. E. (2005). Examining reading development and reading disability in English language learners: Potential contributions from functional neuroimaging. Learning Disabilities Research and Practice, 20, 24-30.

Rasinski, T. V. (2000). Speed does matter in reading. Reading Teacher, 54, 146-151.

Roswell, F.G., Chall, J.S., Curtis, M.E., \& Kearns, G. (2005). Diagnostic assessment of reading. Rolling Meadows, IL: Riverside Publishing.

Samuels, S. J. (1979). The method of repeated readings. The Reading Teacher, 32, 403408.

Samuels, S. J., \& Flor, R. F. (1997). The importance of automaticity for developing expertise in reading. Reading and Writing Quarterly, 13, 107-121.

Snow, C. E., Burns, M. S., \& Griffin, P (Eds.). (1998). Preventing reading difficulties in young children. Washington, DC: National Academy Press.

Stahl, S. A., \& Heubach, K. M. (2005). Fluency-oriented reading instruction. Journal of Literacy Research, 37, 25-60.

Stoddard, K., Valcante, G., Sindelar, P. T., O'Shea, L., \& Algozzine, B. (1993). Increasing reading rate and comprehension: The effects of repeated readings, sentence segmentation, and intonation training. Reading Research and Instruction, 32, 53-65.

Stokes, T. F., \& Baer, D. M. (1977). An implicit technology of generalization. Journal of Applied Behavior Analysis, 10, 349-367.

Tam, K. Y., Heward, W. L., \& Heng, M. A. (2006). A reading instruction intervention program for English-language learners who are struggling readers. The Journal of Special Education, 40, 79-93. 
Therrien, W. J., \& Kubina, R. M. (2006). Developing reading fluency with repeated reading. Intervention in School \& Clinic, 41, 156-160.

Therrien, W. J., Wickstrom, K., \& Jones, K. (2006). Effect of a combined repeated reading and question generation intervention on reading achievement. Learning Disabilities Research \& Practice, 21, 89-97.

U.S. Department of Education (2003). National symposium on learning disabilities in English language learners. Retrieved February 13, 2008, from http://www.ncela.gwu.edu/oela/2003symposium_proceedings.pdf

U.S. Department of Education. (2004). IDEA regulations: Identification of specific learning disabilities. Retrieved February 13, 2008, from http://idea.ed.gov/ explore/view/p/\%2Croot\%2Cdynamic\%2CTopicalBrief\%2C23\%2C

U.S. Department of Education. (2005a). Biennial report to Congress. Retrieved February 13, 2008, from http://ies.ed.gov/doc/biennialrpt05.doc

U.S. Department of Education. (2005b). Institute of Education Sciences, National Center for Education Statistics, Long term trends: Reading. Retrieved February 13, 2008, from http://nces.ed.gov/nationsreportcard/ltt/results2004

Valencia, S. W., \& Pearson, P. D. (1986). Reading assessment: Time for a change. The Reading Teacher, 40, 726-732.

Weinstein, G., \& Cooke, N. L. (1992). The effects of two repeated reading interventions on generalization of fluency. Learning Disability Quarterly, 15, 21-28.

Woodcock, R.W., Mather, N., \& Shrank, K.S. (2001). Woodcock-Johnson III diagnostic reading battery ( $3^{\text {rd }}$ ed.). Rolling Meadows, IL: Riverside Publishing.

Woods, M., \& Moe, A. (2006). Analytical reading inventory (8th ed.). Upper Saddle River, NJ: Prentice Hall. 
APPENDIX A

\section{PARENT/GUARDIAN CONSENT FORM}




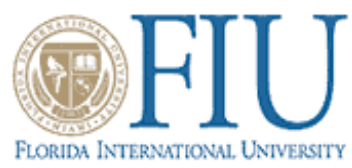

\section{CONSENT TO PARTICIPATE IN A RESEARCH STUDY}

Your child, , is being considered for participation in a research study. The investigator of this study is Katrina Gonzalez Landa and she is a student at Florida International University (FIU). The study will involve students who are English Language Learners who are identified as having Specific Learning Disabilities who are reading below grade level. Your child's participation will require 20 minutes of his or her regular tutoring time.

If you permit your child to be a part of the study, we will integrate the activities into the first 20 minutes of his or her regularly scheduled tutoring time. The research method is similar to what is done during a regular tutoring session. It involves reading a short passage and answering questions about the passage. The exception will be that the researcher will be collecting data on how many words per minute your child reads and how many questions he or she answers correctly at the end of each 20 minute session. The researcher does not expect any harm to your child by being in the study. If he or she becomes frustrated or upset at any point in the study he or she may ask to take a break. You may withdraw your child from the study at any time if you feel in any way uncomfortable. There is no cost to you for your child's participation in the study. This study will provide him or her with additional reading assistance.

The data collected will be identified by a random letter only and not your child's name. All of the information is private and will not be shared with anyone unless required by law. The data will be presented in a graph like table. Your child may ask questions at any time. If you choose not to allow your child to participate no one will be upset with you.

If you would like more information about the research, you may contact Katrina G. Landa at (305) 7783771. You may also contact the faculty advisor at FIU, Dr. Patricia Barbetta at (305) 348-2552. If you would like to ask someone about being a subject in this study you may contact Dr. Patricia Price, the Chairperson of the FIU Institutional Review Board at 305-348-2618 or 305-348-2494.

Thank you for your time.

Katrina Gonzalez Landa

Florida International University

If you have had all of your questions answered to your liking and you would like to be in the study, sign below. Your signature indicates that you will allow/deny your child participate in the in the study.

I give permission for to participate in this study. (Print Child's Name)

Signature of Parent/Guardian Date

I do not give permission for (Print Child's Name) to participate in this study. 


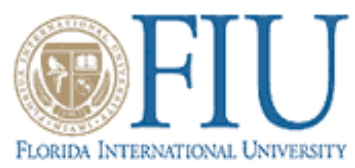

\section{CONSENTIMIENTO PARA TOMAR PARTE EN UN ESTUDIO DE INVESTIGACION}

Su hijo(a), , ha sido tomado(a) en consideracion para participar en un estudio de investigacion. La investigadora de este estudio es Katrina G. Landa, quien es una estudiante al nivel doctoral en la Universidad Internacional de la Florida (FIU). El enfoque del estudio es de estudiantes bilingues que leen por debajo de sus respectivos grados escolares. La participación de su hijo(a) abarcara 20 minutos diarios que estan incluidos en sus sesiones regulares de ensenanza suplementaria.

Si usted permite que su hijo(a) participe en este estudio, integraremos las actividades del estudio en los primeros 20 minutos de sus clases suplementarias. El método de investigación incluye una lectura corta y preguntas acerca del tema repasado durante la lectura. La unica diferencia implementa supervicion por la investigadora cuya tarea será reunir data acerca del numero de palabras leidas por minuto por su hijo(a) y el numero de preguntas contestadas correctamente a la conclusion de cada sesion. La investigadora de este estudio no espera que ningun dano resulte contra su hijo(a) durante el transcurso del estudio Si su hijo(a) se encuentra incomodo(a) durante el lapso del estudio, el/ella podra disculparse de su participacion hasta que el/ella determine apropiado resumir su actividad. Usted puede retirar a su hijo(a) del estudio en cualquier momento si usted lo determina apropiado por cualquier razon. La participacion de su hijo(a) en este estudio resultara en ningun costo de su parte. Este estudio esta disenado con el proposito de proveer ayuda adicional en la lectura de su hijo(a).

La identidad de su hijo(a) se mantendra estrictamente confidencial y solamente representada por una letra asignado en este estudio de investigacion. Toda la información es completamente privada y no será compartida con terceras personas al menos que sea requerido por la ley. Los datos adquiridos en el estudio serán presentados en una forma gráfica y cientifica. Si por alguna razon usted decide no dejar la participacion de su hijo(a) en este estudio de investigacion, tenga en cuenta que no habra ningun tipo de consecuencias negativas o perjudicaciones para su hijo(a).

Si ha usted le gustaria más información acerca de este estudio de investigación, puede llamar a Katrina Gonzalez Landa al (305) 778-3771, o a la Dra. Patricia Barbetta al (305) 348-2552, a su conveniencia. Si a usted le gustaria adquirir mas informacion acerca de este estudio incluyendo la participacion de su hijo(a) puede comunicarse con la directora de los estudios sancionados por el Institutional Review Board (IRB), la Dra. Patricia Price, al (305) 348-2618 o al (305) 348-2494.

Gracias por su atencion.

Katrina Gonzalez Landa, Florida International University

Si usted se encuentra satisfecho(a) con el contenido de este concentimineto y todas sus preguntas han sido adecuadamente contestadas y esta de acuerdo en la participacion de su hijo(a) en este estudio de investigacion por favor tome el tiempo de firmar en la linea de abajo. Su firma tambien indica que usted permitirá a su hijo(a) en participar en el estudio.

Yo doy permiso para

(Imprimir nombre del niño/a) que participle en el estudio.

Firma del Padre/Tutor Fecha

Yo NO doy permiso para

(Imprimir nombre del niño/a) que participle en el estudio.

Firma del Padre/Tutor $\quad$ Fecha 
APPENDIX B

PARTICIPANT ASSENT FORM 


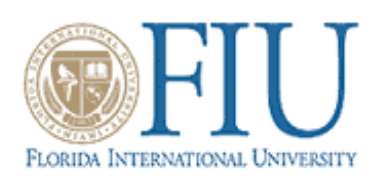

\section{ASSENT TO PARTICIPATE IN A RESEARCH STUDY}

Title: Effects of Repeated Readings on Reading Abilities of English Language Learners with Specific Learning Disabilities.

My name is Katrina Gonzalez Landa and I am a student at Florida International University (FIU). You and some of your classmates are being asked to participate in a research study. This study will look at whether reading something one time or three times helps you to read it faster and understand it better. Being part of this study will give you a chance to practice your reading with me as your tutor.

Your parent/guardian must give you permission to take part in the study. If you do take part, you will be meeting with me for 10 to 20 minutes a day. We will meet in school.

1. You will read a short passage one time or three times.

2. You will answer questions about the passage.

3. I will record the reading and questions.

4. I will listen to the recordings. This will help me count how many words you have read and how many questions you are answering correctly.

There is no charge for being a part of this study. Reading the passages will not harm you in any way. If you get tired or upset, you may ask for a break. I only want you to do your best. Taking part in this study will not help or hurt your grades. You or your parent can ask to stop taking part in the study at any time.

None of the information I collect will have your name on it. Only I will know which ones are your scores. I will write about them in my research paper using a letter (such as student "A"). If you choose not to take part no one will be upset with you.

If you have any questions you can ask anytime. You and your parents may contact me at (305) 778-3771. You may also contact my teacher at FIU, Dr. Patricia Barbetta at (305) 348-2552. If you or your parent feels that you are not being treated fairly in this study, you may contact Dr. Patricia Price, the Chairperson of the FIU Institutional Review Board at 305-348-2618 or 305-348-2494.

If you would like to be in the study, sign below. You will get a copy of this form.

\begin{tabular}{lll}
\hline Sign Here & & Date \\
& Date
\end{tabular}


APPENDIX C

HOME LANGUAGE SURVEY 


\section{Home Language Survey}

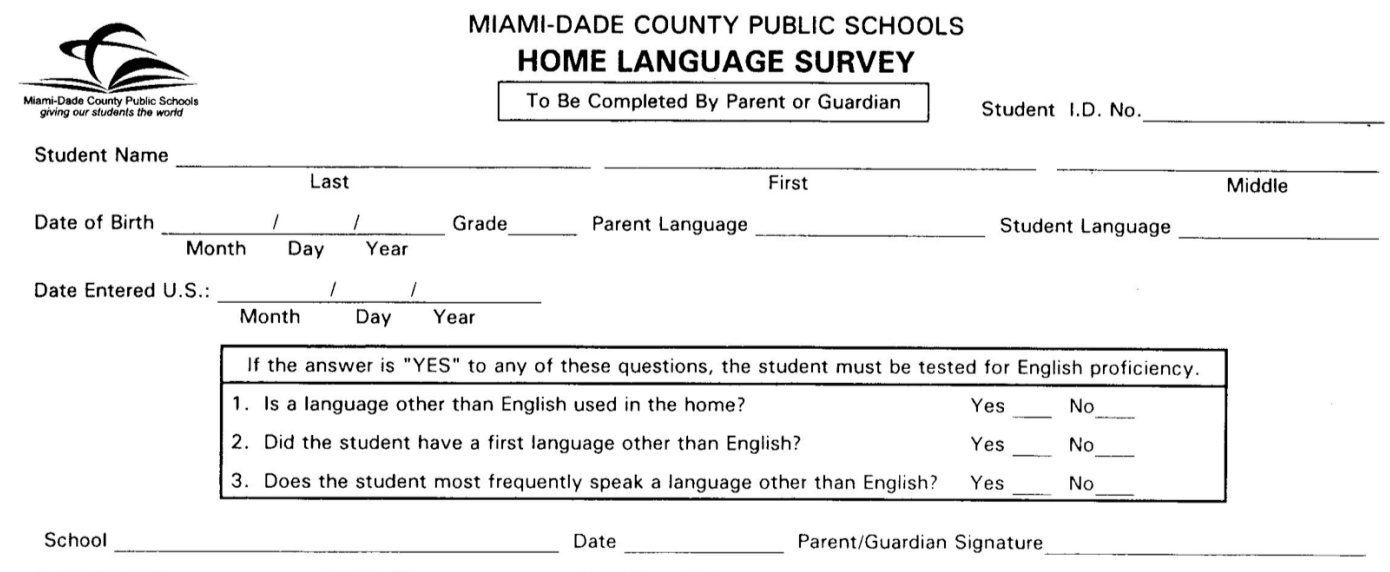

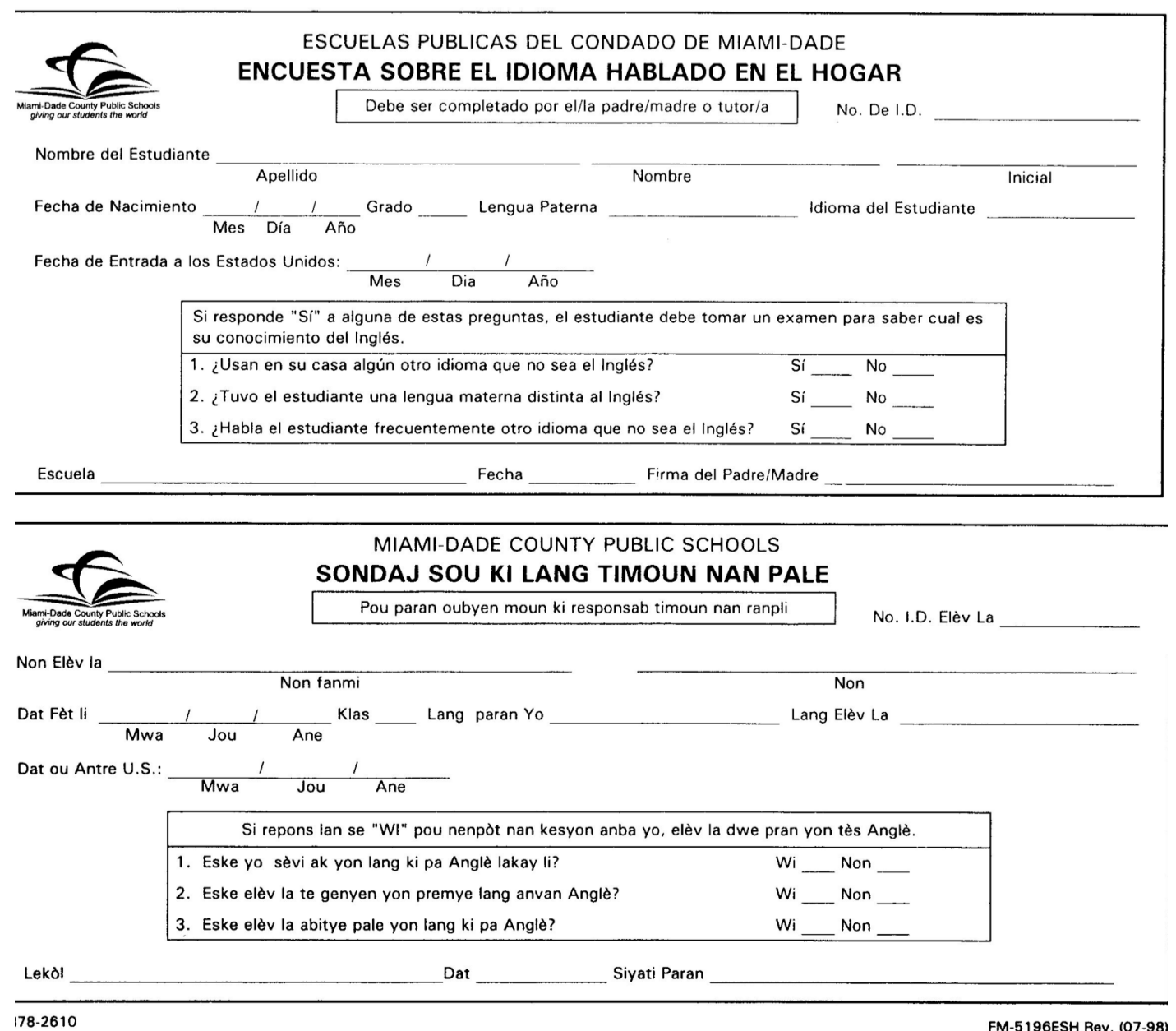




\section{APPENDIX D}

TREATMENT FIDELITY FORM 


\section{Treatment Fidelity Form}

Participant Identification Number:

Observer Filling Out This Form:

Date of Session:

Time:

Phase of Study: (Check One)

Baseline

Maintenance

Intervention

Generalization

Date Of Completion Of This Form:

Time:

Passage \#:

Passage Topic:

Directions: Check off whether the researcher performs the following tasks.

\begin{tabular}{|c|c|c|c|}
\hline \multirow{2}{*}{ Description of Procedure } & \multicolumn{3}{|c|}{ Implemented } \\
\hline & $\begin{array}{l}\mathrm{N} / \\
\mathrm{A}\end{array}$ & Yes & No \\
\hline $\begin{array}{l}\text { 1. The researcher asks the participant: "Can you read the word on the } \\
\text { card?" }\end{array}$ & & & \\
\hline 2. (If participant cannot read the word) The researcher states the word & & & \\
\hline $\begin{array}{l}\text { 3. The researcher asks the participant "Can you use the word in a } \\
\text { sentence?" }\end{array}$ & & & \\
\hline $\begin{array}{l}\text { 4. If the participant can use the word in a sentence, the researcher says } \\
\text { "correct." }\end{array}$ & & & \\
\hline $\begin{array}{l}\text { 5. After stating that the participant is correct the researcher moves on to the } \\
\text { next word. }\end{array}$ & & & \\
\hline $\begin{array}{l}\text { 6. (If the participant cannot use the word in a sentence) The researcher } \\
\text { reads the definition from the back of the card. }\end{array}$ & & & \\
\hline 7. The researcher asks the participant to use the word in a sentence. & & & \\
\hline $\begin{array}{l}\text { 8. (If the participant cannot use the word in a sentence) The researcher uses } \\
\text { the word in a sentence. }\end{array}$ & & & \\
\hline 9. The researcher tells the participant to read the passage. & & & \\
\hline $\begin{array}{l}\text { 10. During this initial reading, when the participant makes an error, the } \\
\text { researcher immediately states the word correctly. }\end{array}$ & & & \\
\hline
\end{tabular}




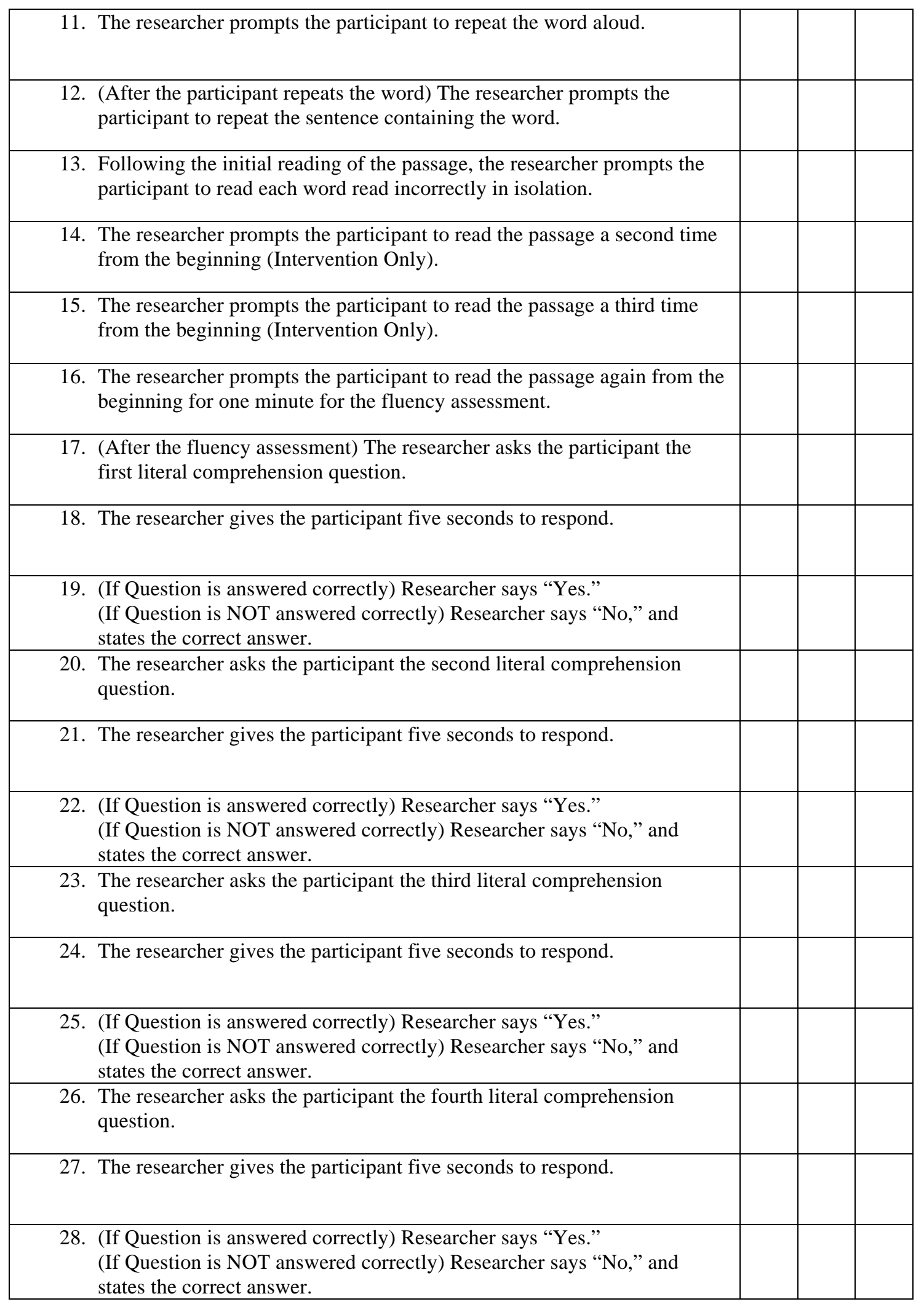


29. The researcher asks the participant the fifth literal comprehension question.

30. The researcher gives the participant five seconds to respond.

31. (If Question is answered correctly) Researcher says "Yes." (If Question is NOT answered correctly) Researcher says "No," and states the correct answer.

32. The researcher issues a closing statement in which she commends the participant for working hard during the session.

\section{Additional Comments:}




\section{APPENDIX E}

INTEROBSERVER AGREEMENT FORM 


\section{Inter-Observer Agreement (IOA) Form}

Participant Identification Letter:

Passage \#: ___ Passage Topic: Session Date:

Directions: This sheet compares the data collected by the researcher with the data collected by the second observer. Using the data sheets independently completed by the researcher and the observer, do a word for word comparison of their marks in each of the following three categories.

Fluency Assessment

\# of words agreed:

\# of words disagreed:

\# Agreements __ $\div$ \# Agreements __ + \# Disagreements __ $\times 100=\ldots \%$ IOA

\section{Errors Per Minute}

\# of errors agreed:

\# of errors disagreed:

\# Agreements $\div$ \# Agreements + \# Disagreements $\ldots+100=$ $\%$ IOA

\section{Types of Errors}

\# of errors agreed:

\# of errors disagreed:

\# Agreements $\div$ \# Agreements + \# Disagreements $\times 100=$ $\% \mathrm{IOA}$

\section{Literal Comprehension Question Assessment}

\# of questions agreed:

\# of questions disagreed:

\# Agreements $\div$ \# Agreements + \# Disagreements $\times 100=$ $\%$ IOA

\section{TOTAL}

Number of Agreements:

Number of Disagreements:

\# Agreements $\div$ \# Agreements + \# Disagreements $\times 100=$ $\%$ IOA 
APPENDIX F

DATA COLLECTION FORM 


\section{Data Collection Form}

Participant Identification Letter:

Person Completing This Form (Check One):

Researcher

Second Observer, Name:

Session Date: Time:

Study Phase: (Check One)

$\begin{array}{ll}\text { Baseline } & \text { Maintenance } \\ \text { Intervention } & \text { Generalization }\end{array}$

Date of Completion of This Form: Time:

\section{Types of Errors}

Directions: Directly on the passage below mark each error in reading with a slash (/). Above each slash, use the following abbreviations to identify the type of error. Place a slash with the word STOP above it in order to identify where the student stopped reading at one minute.

O- Omission

A- Addition

M- Mispronunciation

Passage \#:
S- Substitution

$\mathrm{H}$ - Hesitation more than $5 \mathrm{sec}$

TYPE PASSAGE HERE 
Fluency Assessment:

Directions: Count the number of words read in one minute of reading by counting the words until the word STOP above.

Number of Correct Words Per Minute:

Literal Comprehension Question Assessment

Directions: Mark each of the following questions to determine if the participant's response corresponds to the key.

\begin{tabular}{|l|l|l|}
\hline \multicolumn{1}{|c|}{ Questions } & Correct & Incorrect \\
\hline 1. Type Question Here & & \\
\hline 2. Type Question Here & & \\
\hline 3. Type Question Here & & \\
\hline 4. Type Question Here & & \\
\hline 5. Type Question Here & & \\
\hline
\end{tabular}


VITA

\section{KATRINA G. LANDA}

2000

B.S., Special Education and Psychology

University of Miami

Coral Gables, Florida

2000

Graduate Assistant

Office of Academic Development and Disability

Services

University of Miami

Coral Gables, Florida

2000-2002

Social Studies Teacher of Children with Specific

Learning Disabilities

The Atlantis Academy

Miami, Florida

2002-2005

M.S., Early Childhood Special Education and TESOL University of Miami

Coral Gables, Florida

Pre-kindergarten Special Education Teacher

Ludlam Elementary School

South Miami, Florida

2005-2007

Educational Specialist

Miami-Dade County Public Schools

Miami, Florida

2009

Graduate Assistant College of Education

Florida International University

Miami, Florida

\section{PUBLICATIONS AND PRESENTATIONS}

Nevin, A. et al. (2004). Reviews of Single Subject Research Designs: Applications to Special Education and School Psychology. ERIC Document Reproduction Service No. ED489930

Landa, K. (2004, February). Summary and Critique of a Single Subject Withdrawal Design. Paper presented to Florida International University Graduate Course, EDP 7058, Miami, Florida. 
Landa, K. \& Nevin, A. (2006, April). Pairing Repeated Reading and Systematic Error Correction: Effects in 18 Year Old Students on a Small Caribbean Island. Paper presented at the Florida International University College of Education Research Symposium, Miami, Florida.

Landa, K. (2006, February). Pairing Repeated Reading and Systematic Error Correction: Effects in 18 Year Old Students on a Small Caribbean Island. Paper Presented to Florida International University Graduate Course, EDP 7058, Miami, Florida.

Canal, R. \& Landa, K. (2006, September). Developing, Writing, Administering, Analyzing, and Evaluating Effectiveness of a Community Survey for Program Evaluation. Paper presented at the Florida Adult and Community Educators of Florida Conference, Sanibel Harbor Resort, Ft. Myers, Florida.

Unzueta, C., Landa, K., Leon, E. Tavel, R., Flores, D. (2007, March). Using Inspiration to Promote a UDL Classroom. Workshop presented at Howard D. McMillan Middle School, Miami, Florida.

Landa, K. \& Barbetta, P.M. (2008, October). Using Repeated Readings with English Language Learners with Specific Learning Disabilities. Paper presented at the Florida Council for Exceptional Students, Sarasota Bay, Florida.

Landa, K. \& Barbetta, P.M. (2009, April). Effects of Repeated Readings on English Language Learners with Specific Learning Disabilities. Paper presented at the Florida International University College of Education Research Symposium, Miami, Florida.

\section{CURRENT MEMBERSHIPS}

Golden Key Graduate Honor Society

Alpha Epsilon Lambda Graduate Honor Society

Council for Exceptional Children

\section{AWARDS}

Doctoral Evidence Acquisition Fellowship Recipient (2008). Florida International University, Miami, Florida.

Barnes and Noble Best Student/Professor Paper Recipient (2009), $8^{\text {th }}$ Annual Florida International University College of Education Research Conference, Miami, Florida. 